\title{
Design and Construction of a Fourier Transform Soft X-ray Interferometer
}

\author{
A thesis submitted to the faculty of \\ San Francisco State University \\ in partial fulfillment of the requirements \\ for the degree \\ Master of Science \\ in \\ Physics
}

\author{
by \\ John Spring \\ San Francisco, California
}

May, 2000 


\section{CERTIFICATION OF APPROVAL}

I certify that I have read Design and Construction of a Fourier Transform Soft $X$ ray Interferometer by John Spring and that in my opinion this work meets the criteria for approving a thesis submitted in partial fulfillment of the requirements for the degree: Master of Science in Physics and San Francisco State University.

James Lockhart

Professor of Physics

Adrienne Cool

Professor of Physics

Malcolm Howells

Staff Scientist, LBNL 


\title{
DESIGN AND CONSTRUCTION OF A FOURIER TRANSFORM SOFT X-RAY INTERFEROMETER
}

\author{
John Spring \\ San Francisco State University \\ May, 2000
}

Helium, with its two electrons and one nucleus, is a three-body system. One of the models for investigating correlated electron motion in this system is auto-ionization, produced via double excitation of the electrons. Predictions about the autoionization spectrum of helium have differed from each other and from pre-liminary experimental data. However, previous experiments have not been able to distinguish among the theoretical predictions because their energy resolution is not high enough to resolve the narrow linewidths of quasi-forbidden peaks and the resonances that appear in the highest excited states. Consequently, a team of researchers at Lawrence Berkeley National Laboratory have embarked on a project for building a high-resolution FourierTransform Soft X-ray (or VUV) interferometer (FTSX) to provide definitive data to answer remaining questions about the autoionization spectrum of helium. The design and construction of this interferometer is described in detail below, including the use of a flexure stage to provide the large path length difference necessary for high resolution measurements, the manufacture of $\mathrm{x}$-ray beamsplitters, a description of the software, and the solution to the problems of stick-slip, vibration, and alignment. Current progress of its development is also described, as well as future goals.

I certify that the Abstract is a correct representation of the content of this thesis.

(Chair, Thesis Committee)

(Date) 


\section{ACKNOWLEDGEMENTS}

There are several people I would like to thank for their help in completing this work. Eddie Moler was my mentor in the physics and technical education necessary for building the interferometer. Malcolm Howells and Zahid Hussain directed the work with the wisdom borne of experience and great physical insight. Scott Locklin has been an inspirational proponent of the possibilities that our interferometer holds, and has continued the work with great energy. Jim Lockhart, Adrienne Cool, Malcolm Howells, and Eddie Moler have taken the time from their busy schedules to actually read this thesis and make very constructive comments. (It couldn't have been easy, folks.) Numerous people contributed to the project, including Rob Duarte, Bob McGill, Ted Lauritzen, Troy Barbie, Steve Irick, and all the people at the Advanced Light Source who put the chamber and beamline together.

I would also like to thank my parents and friends for their unstinting and patient support while I slogged my way through the work and thesis, and I would like to especially thank Barbara Klatt for her patience, creativity, support, and everoutward vision, without which my world would have been dimmer. 


\section{TABLE OF CONTENTS}

List of Figures...................................................................................................

List of Appendices ........................................................................................viii

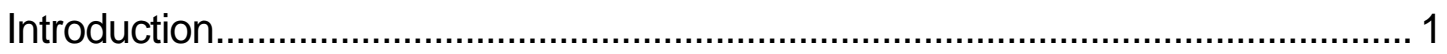

Overview of the Mach-Zehnder Interferometer ...................................................... 5

Motivation for choosing the Mach-Zehnder configuration ........................................ 5

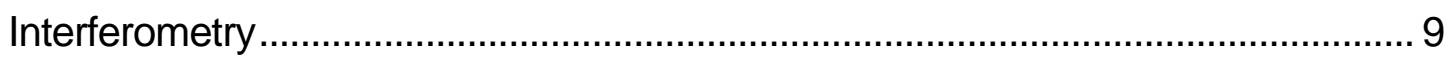

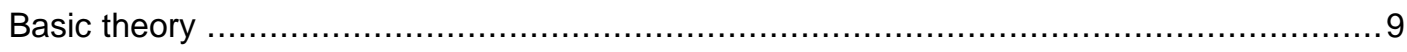

Determining Optical Component Tolerances .......................................................... 11

Description of Mach-Zehnder interferometer ..................................................22

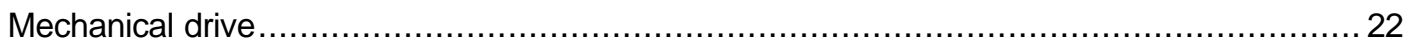

X-ray Optics

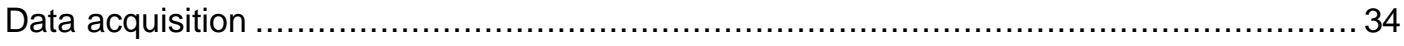

Measurement and rectification of tilt in stage movement....................................41

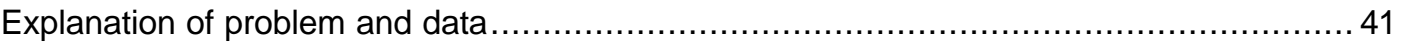

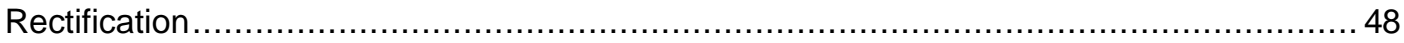

Measurement and rectification of vibration and stick-slip.................................50

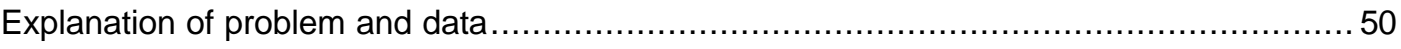

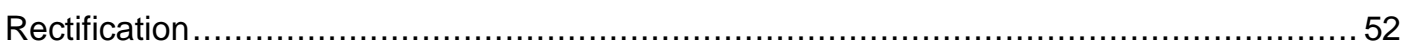

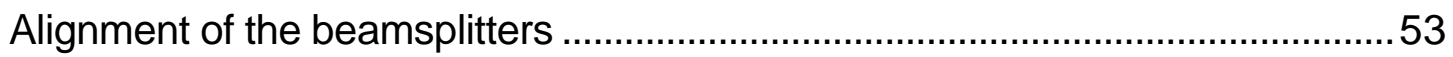


Conclusions 56

Appendix 1: Derivation of Geometric Factor Used in Calculating Path Length Difference .....................................................................................................

Appendix 2: Software Descriptions .............................................................59

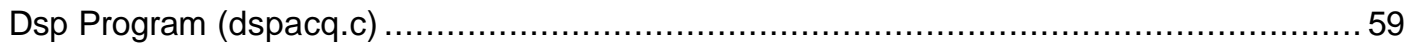

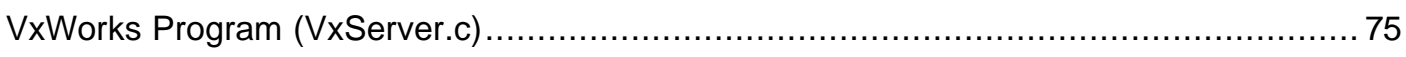




\section{List of Figures}

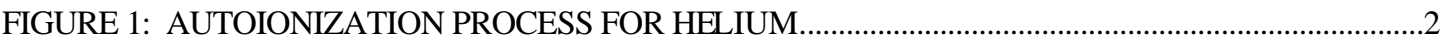

FIGURE 2: OPTICAL LAYOUT OF BEAMLINE 9.3.2 AT THE ALS.............................................................

FIGURE 3: PHOTOGRAPH OF THE SOFT X-RAY INTERFEROMETER ....................................................

FIGURE 4: DIAGRAMS OF THE PATHS OF THE SPLIT BEAMS THROUGH THE X-RAY

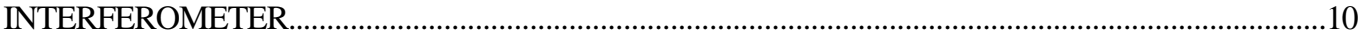

FIGURE 5: SIMPLIFIED RAY DIAGRAM FOR DESCRIBING SPHERICAL ERRORS................................14

FIGURE 6: RAY DIAGRAM FOR TILT ERRORS...................................................................................17

FIGURE 7: SCHEMATIC DIAGRAM FOR DESCRIBING TILT ERRORS FOR ONE OF THE X-RAY

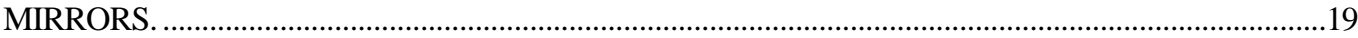

FIGURE 8: SCHEMATIC DIAGRAM FOR TILT ERRORS. THE MIRROR HAS BEEN ROTATED........20

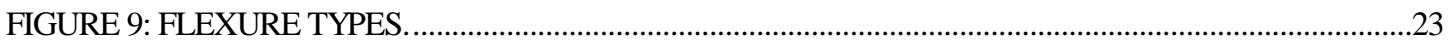

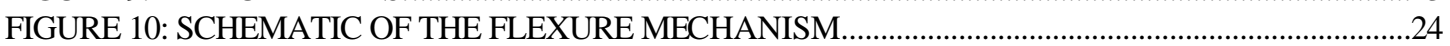

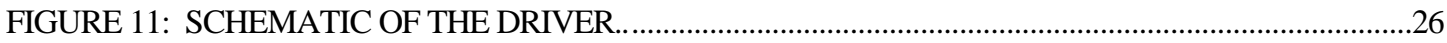

FIGURE 12: BEAMSPLITTER SCHEMATIC...……………………………………………………....2

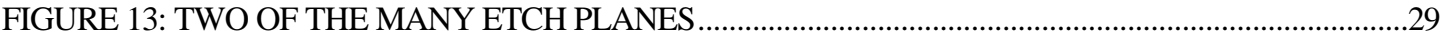

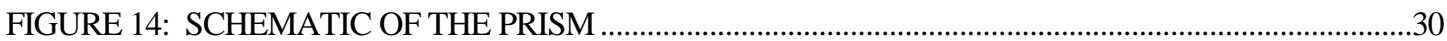

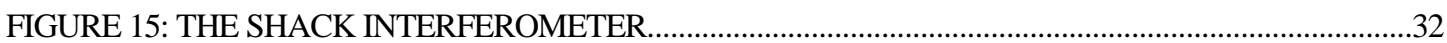

FIGURE 16: TEST SETUP FOR DETERMINING PERPENDICULARITY OF A SIDE OF THE MIRROR

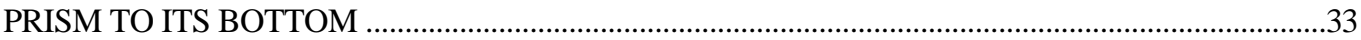

FIGURE 17: SCHEMATIC OF FLEXURE MECHANISM, SHOWN DEFORMED. …………......................42

FIGURE 18: SCHEMATIC FOR TILT ANALYSIS. ...................................................................................43

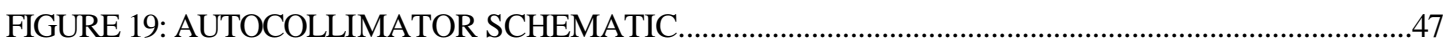

FIGURE 20: CORRECTION OF STAGE TILT WITH TAPER PIN ADJUSTMENT ……………………......49

FIGURE 21: EXPANDED PLOT OF STAGE TILT AFTER CORRECTION. THE PITCH ERROR HAS BEEN REDUCED TO 0.38 MRAD, RMS...................................................................................50

FIGURE 22: THE STAGE IS MOVING IN A NEGA TIVE DIRECTION AT -31.3 MM / SEC........................51

FIGURE 23: THESE ARE THE SAME DATA (OF FIGURE 22) AFTER THEY WERE FIT WITH A LINE52 FIGURE 24: POWER SPECTRUM OF MECHANICAL VIBRATIONS ……………….................................52

FIGURE 25: CONTACT POINTS ON A BEAMSPLITTER. ………………………………………….......54

FIGURE 26 ALIGNMENT SCAN USING INCOHERENT NEON LIGHT........................................................55

FIGURE A: PATH LENGTH CHANGE DUE TO MOVING ONE OF THE MIRRORS...................................58 


\section{List of Appendices}

APPENDIX 1: DERIVATION OF GEOMETRIC FACTOR USED IN CALCULATING PATH LENGTH DIFFERENCE

APPENDIX 2: SOFTWARE DESCRIPTIONS

59

viii 


\section{Introduction}

Helium has two electrons and a nucleus, making it a three-body system. As such, it is interesting for theoretical atomic physicists, who have made predictions about the autoionization spectrum resulting from double excitation of the electrons ${ }^{1,2}$.

Autoionization is a process in which both electrons are excited to energy levels above the ground state; during the subsequent interaction of the continuum and the Rydberglike states of the two electrons' wavefunction, one of the electrons is ionized.

Specifically, the "inner" electron is excited into a shell N 2, and the "outer" electron into a shell $n>N$. More than thirty years ago, R.P. Madden and K. Codling discovered that there is strong mixing among the doubly-excited states of helium. ${ }^{1}$ That is, while a simple model of the two electrons behaving independently would suggest an absorption spectrum including separate lines for the $2 s$ and $2 p$ series, they observed only one series. In the paper following that one J.W. Cooper, U. Fano, and F. Prats suggested some properties and a theory for correlated electron motion in doubly-excited helium. ${ }^{2}$

\section{Need for higher resolution measurements}

Theoretical predictions of the linewidths of the absorption spectrum of doubly-excited helium have not agreed with each other nor adhered closely to previous 


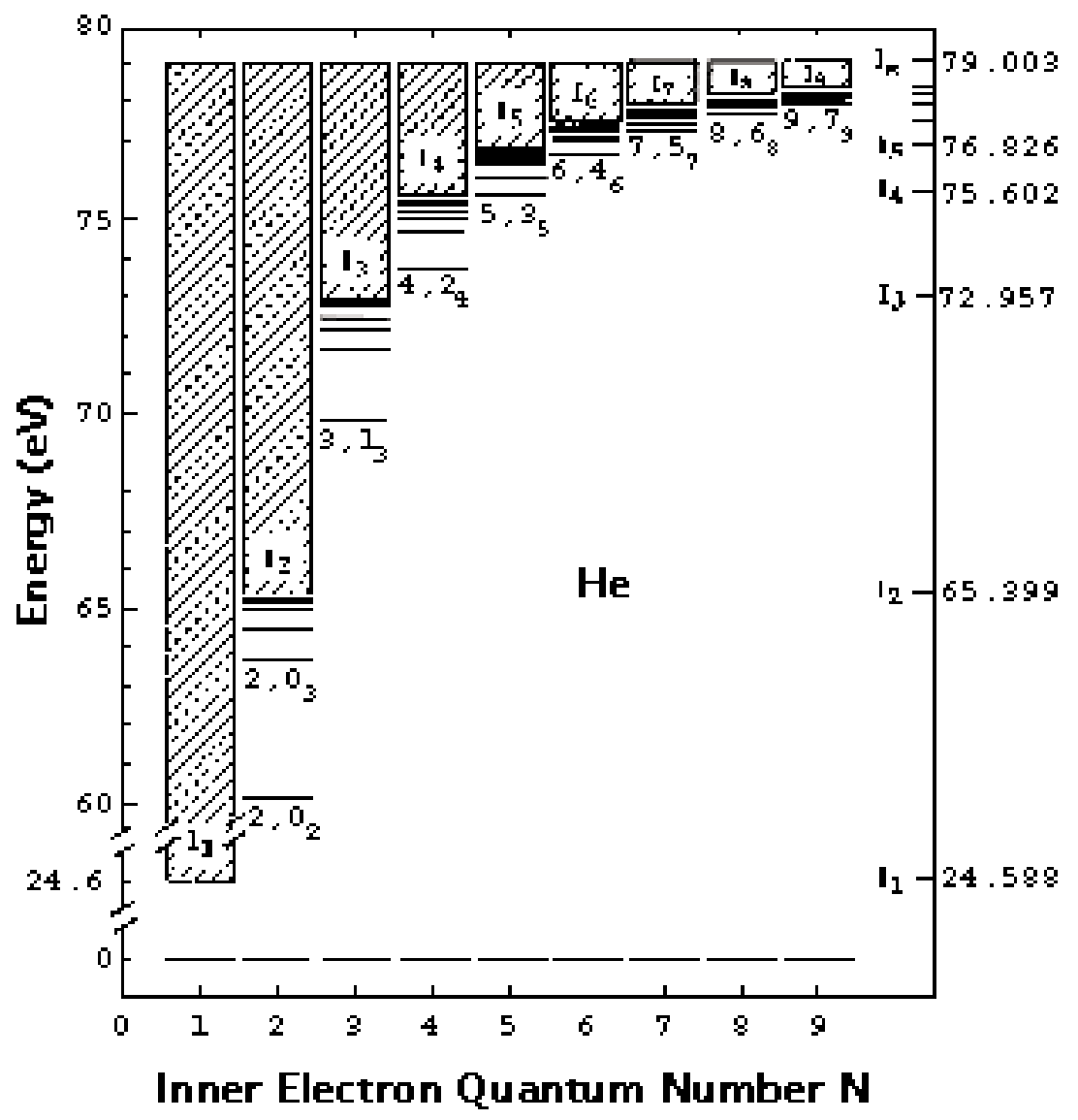

Figure 1: Autoionization process for helium. Both electrons are excited into higher energy states; the autoionization spectrum results from the interaction of the discrete Rydberg-like states and the continuum.

measurements using transmission gratings. For instance, the linewidth for the ${ }_{2}(-1,0)_{3}^{0}$ emission was calculated to be $4 \times 10^{-5} \mathrm{meV}$ by $\mathrm{Wu}$ and $\mathrm{Xi}^{3}, 3 \times 10^{-2} \mathrm{meV}$ by Macías, et al ${ }^{4}$ and estimated to be $<5 \times$ by $10^{-2} \mathrm{meV}$ by Domke, et al. ${ }^{5}$, et al. (Doubly-excited states of helium may by labeled according to the notation developed by C. D. Lin, ${ }^{n(K, T)_{N}^{A}}$, where $n$ is the index of the "outer" electron, $N$ is the "inner" electron, $\mathrm{K}[=\mathrm{N}-1-\mathrm{T}, \mathrm{N}-3-\mathrm{T}, \ldots,-(\mathrm{N}-1-\mathrm{T})]$ and $\mathrm{T}(=0,1)$ are quantum number describing the angular correlations, and $\mathrm{A}(=+1,-1,0)$ describes the radial correlation. ${ }^{6}$ ) Since the best resolution attained so far, at the Advanced 
Light Source, was $E / \delta E=64,000^{7}$, it is clear that the higher resolution attainable with this interferometer is required to answer these questions.

\section{Overview of Beamline 9.3.2}

The synchrotron light this experiment uses comes from a bending magnet at the Advanced Light Source at Lawrence Berkeley National Laboratory. Following is a description of the beamline, synopsized from the $\mathrm{PhD}$ thesis of W.R.A. Huff ${ }^{8}$

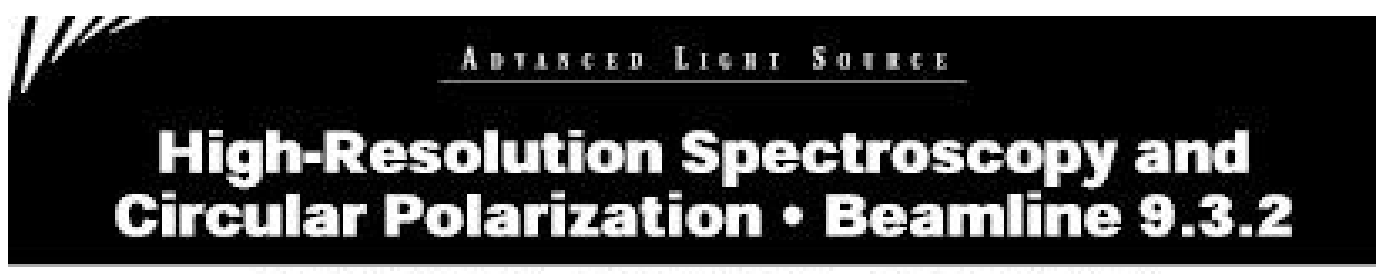

Berkeley Lab. University of calforata

\section{Beamiline Speoiflioations}

\begin{tabular}{|c|c|c|c|c|c|}
\hline $\begin{array}{l}\text { Phatan } \\
\text { Energy Range } \\
\text { (w) }\end{array}$ & $\begin{array}{c}\text { Photan } \\
\text { Flux } \\
\text { ishobanabould.1saw) }\end{array}$ & $\begin{array}{l}\text { Spoctral } \\
\text { Resolution } \\
\text { |ElaE| }\end{array}$ & $\begin{array}{l}\text { Spot } \\
\text { Size } \\
\text { (mit) }\end{array}$ & Polarization & Avalsbility \\
\hline $30-1500$ & 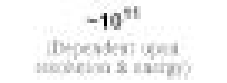 & 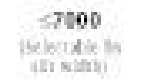 & $0.5 \times 1$ & 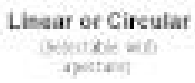 & Now \\
\hline
\end{tabular}

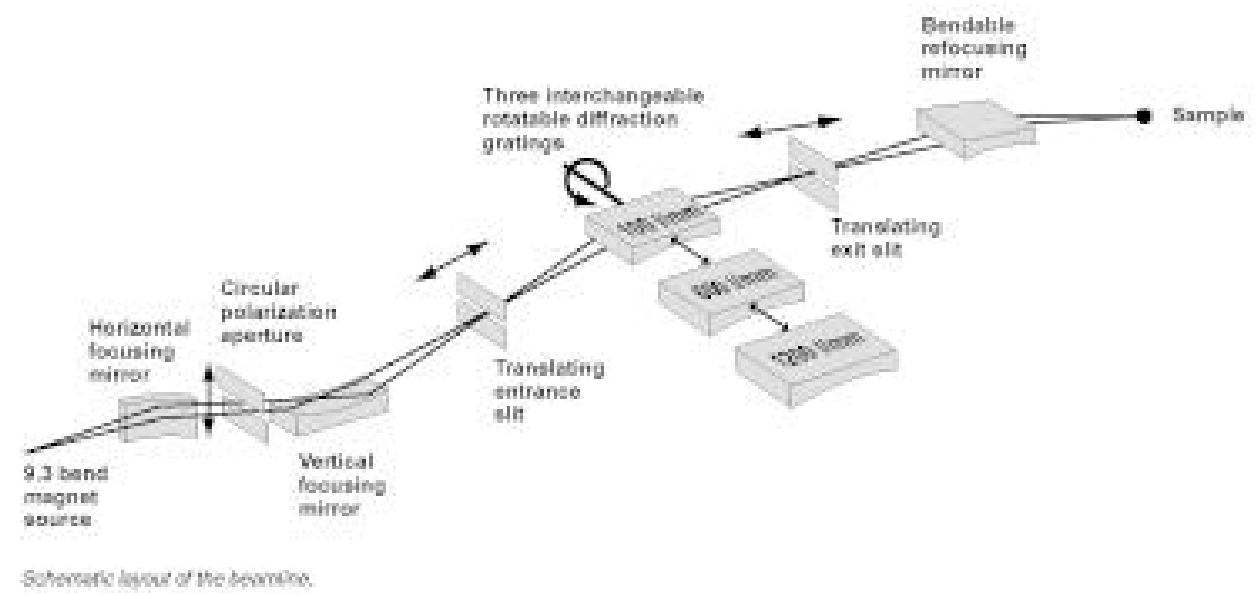

Figure 2: Optical layout of Beamline 9.3.2 at the ALS. 
Light exits the synchrotron and strikes a cylindrical horizontal focusing mirror, whose focus is at the exit slit. The light then goes through a circular polarization-selection aperture (CPA). Bending magnet radiation is linearly polarized in the horizontal plane of the centroid of the beam; above and below the horizontal plane, the vertical component of polarization is nonzero, producing left and right circular polarization, respectively. Thus, if the CPA is centered on the beam, horizontal linearly polarized light is transmitted. Moving the CPA above the beam produces left elliptical polarization that becomes more circular the farther the CPA is moved. Similar motion below the beam produces right elliptical polarization.

Continuing downstream, the beam encounters the vertical focusing mirror, whose focus is the entrance slit. Next, the beam encounters the entrance slit. The entrance and exit slits determine the resolution of the beam according to the equation

$$
\Delta \lambda_{\mathrm{sW}}=\sqrt{\left(\frac{\mathrm{W}_{\mathrm{S} 1} \mathrm{~d} \cos \alpha}{\mathrm{mr}_{\mathrm{S} 1}}\right)^{2}+\left(\frac{\mathrm{W}_{\mathrm{S} 2} \mathrm{~d} \cos \beta}{\mathrm{mr}_{\mathrm{S} 2}}\right)^{2}}
$$

where $W_{S 1}=$ entrance slit width

$$
\begin{aligned}
W_{\mathrm{S} 2} & =\text { exit slit width } \\
d & =\text { groove spacing if monochromator grating } \\
\alpha & =\text { incident angle of beam to grating } \\
\beta & =\text { diffracted angle of beam from grating } \\
m & =\text { diffraction order } \\
r_{\mathrm{S} 1} & =\text { distance from entrance slit to grating } \\
r_{\mathrm{S} 2} & =\text { distance from grating to exit slit }
\end{aligned}
$$

The beam next encounters the diffraction grating which, with the entrance and exit slits, constitutes the monochromator. The energy of the transmitted beam is 
selected from the energy band entering the monochromator by adjusting the angles $\alpha$ and $\beta$ and order $m$ in the equation

$$
\pm m \lambda=d(\sin \alpha+\sin \beta)
$$

where $\lambda$ is the wavelength of the desired energy.

\section{Overview of the Mach-Zehnder Interferometer}

\section{Motivation for choosing the Mach-Zehnder configuration}

We chose a Mach-Zehnder configuration, and modified its original arrangement of mirrors from a square to a rhombus. This was done to present grazing-incidence angled surfaces to the x-ray beam, which would otherwise be absorbed by surfaces at steeper angles. Two considerations led us to choose the Mach-Zehnder interferometer over other suitable optical systems:

1. Grazing-incidence requirement. X-rays at high incidence angles are absorbed by any material used in optics, so an optical system whose geometry exploits low incidence angles has a higher throughput. For instance, the Michelson interferometer has a single beamsplitter and two retroreflectors. The retroreflectors require 90 incidence angles, so they would absorb most of the x-rays. Other configurations of beamsplitters and mirrors suffer from similar limitations.

2. High energy resolution. This is the most important reason. Previous highresolution studies in this energy regime were carried out using reflection-grating monochromators. (It is a spherical-grating monochromator that was used to get the current highest resolution measurements of the autoionization spectrum of helium.) For gratings resolution is determined by entrance and exit slit widths, the groove spacing of the grating, the distance from the entrance slit to the grating, and the distance from the grating to the exit slit. For interferometers, the energy resolution 
is determined by the path-length difference between the two beams. (Actually, the resolution is defined by the equation $\delta \mathrm{E}=\frac{\mathrm{hc}}{\Delta \mathrm{x}}$ where $\mathrm{x}$ is the path-length difference (PLD). Resolving power is a unitless measure of the quality of an optical system, defined by $\mathrm{R}=\frac{\mathrm{E}}{\delta \mathrm{E}}$.) The resolving power necessary to measure the narrow linewidths of the quasi-forbidden peaks $\left(\sim 10^{-5} \mathrm{eV}\right)$ is on the order of $10^{6}$ To get this resolving power for $\mathrm{x}$-rays with an energy of 65 eV requires a PLD of

$$
\Delta \mathrm{x}=\frac{\mathrm{hc}}{\delta \mathrm{E}}=\frac{\mathrm{hcR}}{\mathrm{E}}=\frac{(1.24 \mathrm{eV}-\mu \mathrm{m})\left(10^{6}\right)}{65 \mathrm{eV}} \cong 20,000 \mu \mathrm{m}=2.0 \mathrm{~cm}
$$

Introducing a $2 \mathrm{~cm}$ PLD while maintaining alignment of the separate beams (the physical setup and optical tolerances will be described below) imposed unprecedented technical requirements on the motion system . This range of travel is several orders of magnitude beyond the range of piezoelectric ceramics, and a motion system that uses a lead screw is simply too jerky and imprecise in its motion. Flexure stages have proven themselves able to accomplish straight and smooth motion for travel in the range $10^{-2}-10^{-1} \mathrm{~cm}$, so we used their design as a model for our motion system. 


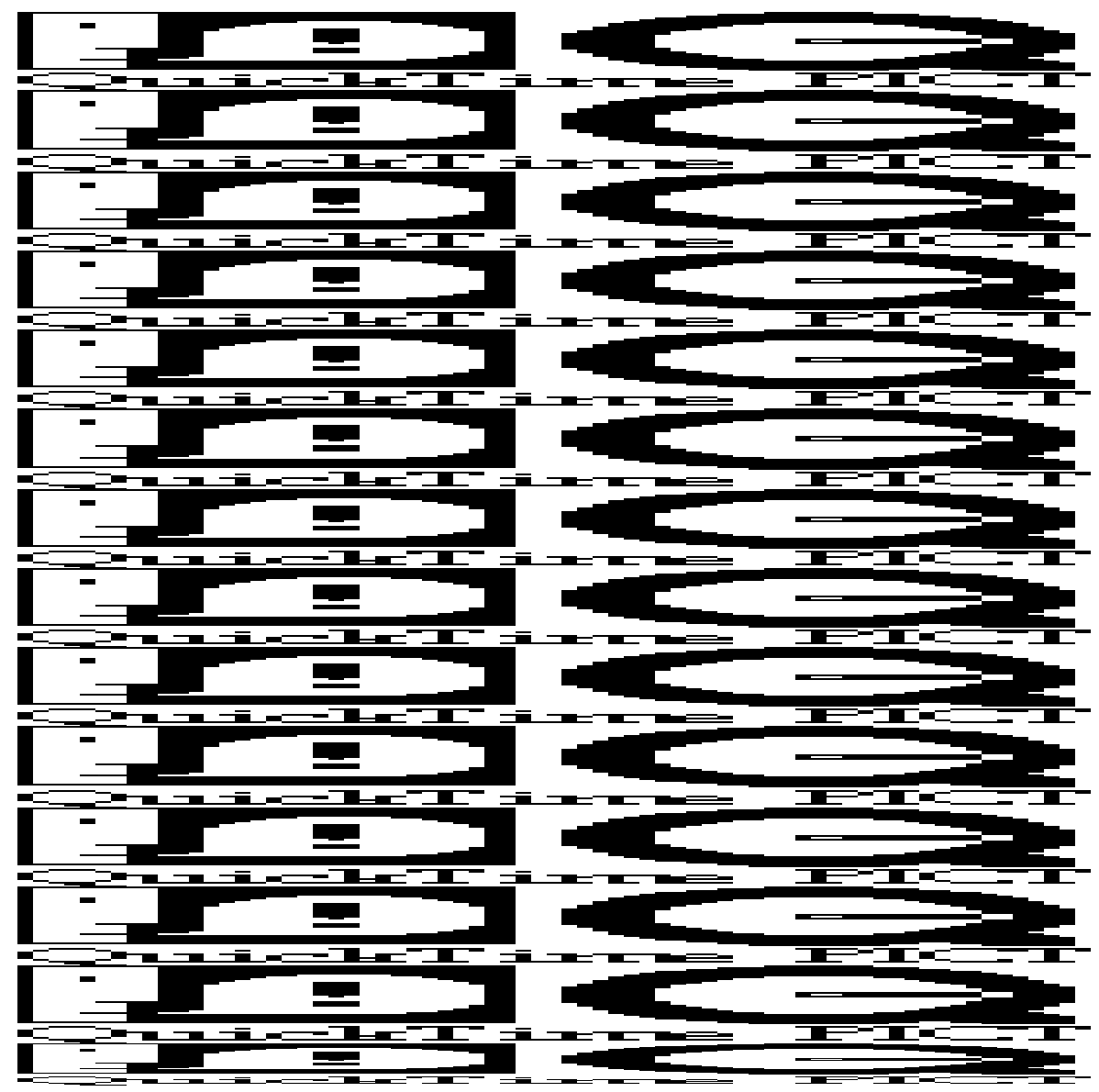

Figure 3: Photograph of the soft x-ray interferometer. The driveshaft at lower left pushes the flexure stage in the center. The stage carries the mirrors. The position of the stage is measured by the laser interferometer above. The $x$-ray beam itself enters from left center and strikes the upstream beamsplitter at an angle of $20^{\circ}$. The subsequent paths of the two beams is indicated; one is reflected from the upper pair and one from the lower pair of mirrors, and then they are recombined at the downstream beamsplitter. The laboratory reference frame used throughout this paper is defined as a right-handed Cartesian coordinate system with the z-axis in the direction of stage travel (roughly from lower left toward upper right in the photograph), the $x$-axis in the vertical direction (out of the page in the photo), and the $y$-axis orthogonal to $x$ and $z$ (toward the lower right in the photo). 


\section{Brief description of the Mach-Zehnder interferometer}

The Mach-Zehnder interferometer comprises three subsystems: the x-ray optics, the mechanical drive, and the data acquisition and control system (see figure 3). The $x$ ray optics comprise the mirrors and beamsplitters. The purpose of the optics is to split the x-ray beam into two beams, introduce a path length difference, and then coherently recombine them. The four mirrors are supported by a flexure stage (described below) and therefore move through its $1.5 \mathrm{~cm}$ range. The beamsplitters are fixed to the frame of the flexure mechanism. In order for the separated beams to recombine coherently, the optics must be aligned to within $\sim 1 \mu \mathrm{rad}$. The reflecting surfaces of the beamsplitters should be coplanar, and opposing sides of the rhombus-shaped mirror assembly should be parallel. This alignment is done near zero path length difference (ZPD) with the stage at rest. It became apparent that manual alignment, by turning screws, was too crude, so we added computercontrolled picomotors to automatically step through the small angles necessary for proper alignment.

The mechanical drive comprises the flexure mechanism, a large hydraulic driver, and an aluminum driveshaft connecting the two. Its purpose is to push and pull the $x$ ray mirrors through a total distance of about $1.5 \mathrm{~cm}$, with a minimum of vibration and pitch angle. At the center of the flexure mechanism is a stage, a block of steel 2.62" $x 4.75 " \times 2.00 "$, that supports the mirror assembly. The entire flexure mechanism was cut from a single piece of steel using electric-discharge machining (EDM). The hydraulic driver was designed to push against the rather large spring force of the flexure mechanism, but at the same time could not introduce vibrations, into the mirrors, whose frequencies were near those of the interference fringes as they moved by the x-ray detector.

The data acquisition system comprises instrumentation and software for acquiring three main channels of data: stage position, $x$-ray signal, and $x$-ray reference. The control system controls the piston and beamsplitter alignment via picomotors. 


\section{Interferometry}

\section{Basic theory}

An interferometer works by splitting a beam of light, sending the component beams along two separate paths, and then coherently recombining them. Coherence means that there is a constant phase between the recombining beams across a cross-section of the reconstituted beam.

If we have a plane wave that has been split into two plane waves of equal amplitude given by

$$
\begin{aligned}
& E_{1}=E_{0}(\mathbf{x}) e^{-i(k " x-\omega t)} \\
& E_{2}=E_{0}(\mathbf{x}) e^{-i(k " x-\omega t+\delta)}
\end{aligned}
$$

they will interfere with a phase difference $\delta$, produced by an optical path difference. The intensity of the sum of the two waves is given by

$$
\begin{aligned}
\mathrm{I} & =\left|\mathrm{E}_{1}+\mathrm{E}_{2}\right|^{2} \\
& =\left|\mathrm{E}_{0}(\mathbf{x}) \mathrm{e}^{-\mathrm{i}(\mathbf{k} \mathbf{x}-\omega \mathrm{t})}\left(1+\mathrm{e}^{-\mathrm{i} \delta}\right)\right|^{2} \\
& =2 \mathrm{E}_{0}{ }^{2}(\mathbf{x})(1+\cos \delta) \\
& =2 \mathrm{E}_{0}{ }^{2}(\mathbf{x})[1+\cos (2 \pi \sigma \mathrm{x})] \\
& =\mathrm{B}(\sigma)[1+\cos (2 \pi \sigma \mathrm{x})]
\end{aligned}
$$

where $x$ is the optical path difference, $\sigma=1 / \lambda$ is the wavenumber of the $x$-rays, and $\mathrm{B}(\sigma)=4\left[\mathrm{E}_{0}^{2}(\mathrm{x})\right]=4 \mathrm{~S}(\mathrm{t})$ is the time-averaged energy flux through the system. For a grazing incidence Mach-Zehnder interferometer whose mirrors are canted at an angle of one-half the incidence angle, the path length difference is given by

$$
x=4 \Delta y \sin \alpha
$$

where the incidence angle $\alpha$ is measured from the plane of reflection of the first beamsplitter, and $\Delta y$ is the distance the mirrors move perpendicular to the line connecting the beamsplitters (see Appendix 1 for derivation). 
Figure 4: Diagrams of the paths of the split beams through the $x$-ray interferometer. The upper diagram gives the path lengths when the interferometer is at the high limit of traversal, and the lower diagram is at the low limit. The total path difference between the two beams when the stage moves from the high limit to the low limit is $2 \times(300.55 \mathrm{~mm}-290.13 \mathrm{~mm})=$ $20.84 \mathrm{~mm}$. For $x$-rays with a wavelength of $20 \mathrm{~nm}$, this corresponds to about $1,040,000$ waves. Also indicated is the reference frame that will be used throughout this paper to describe the interferometer. It is a right-handed system in which the $z$-coordinate is in the direction of travel of the stage, $x$ is directed vertically, out of the page, and $y$ is to the right. Roll, yaw, and pitch refer to rotations around the $z, x$, and y axes, respectively. "Tilt" refers to any rotation.

For each component of light $d \sigma$, the intensity is given by

$$
I \mathrm{~d} \sigma=\mathrm{B}(\sigma) \mathrm{d} \sigma+\mathrm{B}(\sigma) \cos (2 \delta \sigma \mathrm{x}) \mathrm{d} \sigma
$$

so that the total intensity for broadband light is given by the integral

$$
I(x)=\int_{0}^{\infty} B(\sigma) d \sigma+\int_{0}^{\infty} B(\sigma) \cos (2 ð \sigma x) d \sigma
$$

At zero path length difference, $x=0$, so 


$$
\mathrm{I}(0)=2 \int_{0}^{\infty} B(\sigma) \mathrm{d} \sigma
$$

and

$$
\begin{aligned}
I(x) & =\frac{I(0)}{2}+\int_{0}^{\infty} B(\sigma) \cos (2 ठ \sigma x) d \sigma \\
& =\frac{I(0)}{2}+F(x)
\end{aligned}
$$

where

$$
F(x)=\int_{0}^{\infty} B(\sigma) \cos (2 ð \sigma x) d \sigma .
$$

The constant I(0)/2 appears in an interferogram as a dc offset, and is proportional to the flux through the system. The integral is called the interference function, and is the position-dependent component of the intensity. It is also the Fourier transform of $\mathrm{B}(\sigma)$ so we may perform the inverse Fourier transform to get

$$
B(\sigma)=\int_{0}^{\infty} F(x) \cos (2 ð \sigma x) d x .
$$

\section{Determining Optical Component Tolerances}

The sensitivity of the interferometer depends on the ability of the detector to see high contrast fringes over the greatest range of stage travel possible.

Following Chamberlain',

$$
I(x)=\int_{0}^{\infty} B(\sigma) d \sigma+\int_{0}^{\infty} B(\sigma) \cos (2 \delta \sigma x) d \sigma .
$$

The most general expressions for the energy flux $B$ and path length difference $x$ are $\mathrm{B}=\mathrm{B}(\sigma, \mathrm{u}, \mathrm{v}, \mathrm{t})$ and $\mathrm{x}=\mathrm{x}(\mathrm{u}, \mathrm{v}, \mathrm{t})$ where $\mathrm{u}, \mathrm{v}$ are the coordinates in a plane perpendicular to the beam direction. If we assume that the flux is time-independent and uniform in space (i.e. a plane wave -- a reasonable approximation given that the exit slit of the monochromator is $\sim 3 \mathrm{~m}$ from the interferometer), B depends only on $\sigma$, and we can write the power distribution as $\frac{\mathrm{B}(\sigma) \mathrm{d} \sigma}{\mathrm{A}}$ where $\mathrm{A}$ is the area of the incoming beam. Thus for a lossless interferometer the contribution to the total power at the detector from an element $d A=d u d v$ at $(u, v)$ is

$$
\mathrm{dI}(\mathrm{x})=\int_{0}^{\infty} \mathrm{d} \sigma \frac{\mathrm{B}(\sigma)}{\mathrm{A}}[1+\cos (2 \pi \sigma \mathrm{x}(\mathrm{u}, \mathrm{v}))] \mathrm{dA}
$$

and the total power is found by integrating over the area of the interfering beams 


$$
\mathrm{I}(\mathrm{x})=\int_{0}^{\infty} \mathrm{d} \sigma \frac{\mathrm{B}(\sigma)}{\mathrm{A}} \iint_{\mathrm{A}}[1+\cos (2 \pi \sigma \mathrm{x}(\mathrm{u}, \mathrm{v}))] \mathrm{dudv}
$$

where $x(u, v)$ is the path difference of the rays at position $(u, v)$ on the detector. In a perfectly aligned system with distortion-free optics, the path length difference $x$ would not depend on $u$ and $v$. In other words, plane wave in -> plane wave out. If, however, a small increment $\delta$ to the path length difference is produced by misalignment or distortions of the optics, we can write $x(u, v)=x+\delta(u, v)$. Substituting into the cosine term of the above equation gives

$$
\begin{aligned}
\cos 2 \pi \sigma x(u, v) & =\cos 2 \pi \sigma[x+\delta(u, v)] \\
& =\cos (2 \pi \sigma x) \cos (2 \pi \sigma \delta)+\sin (2 \pi \sigma x) \sin (2 \pi \sigma \delta) \\
& =\cos (2 \pi \sigma x) \cos (2 \pi \sigma \delta)
\end{aligned}
$$

since $\delta<1$. Substituting into the power equation (1) gives

$$
\begin{aligned}
I(x) & =\int_{0}^{\infty} \mathrm{d} \sigma \frac{\mathrm{B}(\sigma)}{\mathrm{A}} \iint_{\Lambda}[1+\cos (2 \pi \sigma \mathrm{x}) \cos (2 \pi \sigma \delta)] d u d v \\
& =\int_{0}^{\infty} \mathrm{B}(\sigma) \mathrm{d} \sigma+\int_{0}^{\infty} \mathrm{B}(\sigma) \cos (2 \pi \sigma \mathrm{x}) \mathrm{d} \sigma\left[\frac{1}{\mathrm{~A}} \iint_{\mathrm{A}} \cos (2 \pi \sigma \delta) d u d v\right] \\
& =\int_{0}^{\infty} \mathrm{B}(\sigma) \mathrm{d} \sigma+\int_{0}^{\infty} \mathrm{B}(\sigma) \mathrm{D}(\sigma) \cos (2 \pi \sigma \mathrm{x}) \mathrm{d} \sigma
\end{aligned}
$$

where

$$
D(\sigma)=\frac{1}{A} \iint_{A} \cos (2 \pi \sigma \delta) d u d v
$$

is the spectral distortion factor whose value determines the visibility of the fringes. We chose a value $D(\sigma)>0.9$, giving a $90 \%$ visibility of the fringes. This value was chosen to set a practical limit on the tolerances required of the various manufacturers of the optical and mechanical components of the interferometer (see below). $R=$ radius of system aperture $=1 \mathrm{~mm}$ and $\sigma=1 / \lambda=10^{6} \mathrm{~cm}^{-1}$. (The wavelength was set at $10 \mathrm{~nm}$ rather than 20 because we want the upper energy limit to be $120 \mathrm{eV}$. The $10 \mathrm{~nm}$ wavelength forces tighter tolerances and gives a wide margin of error.)

There are several possible causes for the two beams to not be parallel to one another at the detector. (Since the wavelength is so short and we are measuring the 
total intensity over the whole detector, the two beams must have constant phase over the dimensions if the detector.) The errors fall under two categories: spherical and tilt errors. If one beam is curved with respect to the other, then even if they interfere properly on one side of the detector, by the time we get to the other side, the curvature in the first beam will produce a path error that could cancel the measurement of the interference. Spherical errors arise from the x-ray source not producing parallel $x$-rays and from nonplanar optical surfaces. Tilt errors, in which both beams are plane waves but are tilted with respect to one another, may be subdivided into two categories: slope errors, due to poor surfaces of the optical components, and alignment errors, which arise from nonparallel mirrors, tilt of the mirrors with respect to the beamsplitters during stage motion, and misalignment of the beamsplitters with respect to the mirrors. All the possible causes except the $x$ ray source and beamsplitter misalignment would be due to interferometer manufacturing errors. It was necessary to accurately calculate the greatest tilt and sphericity errors we could tolerate and distribute them among the optical components reasonably.

\section{Spherical errors}




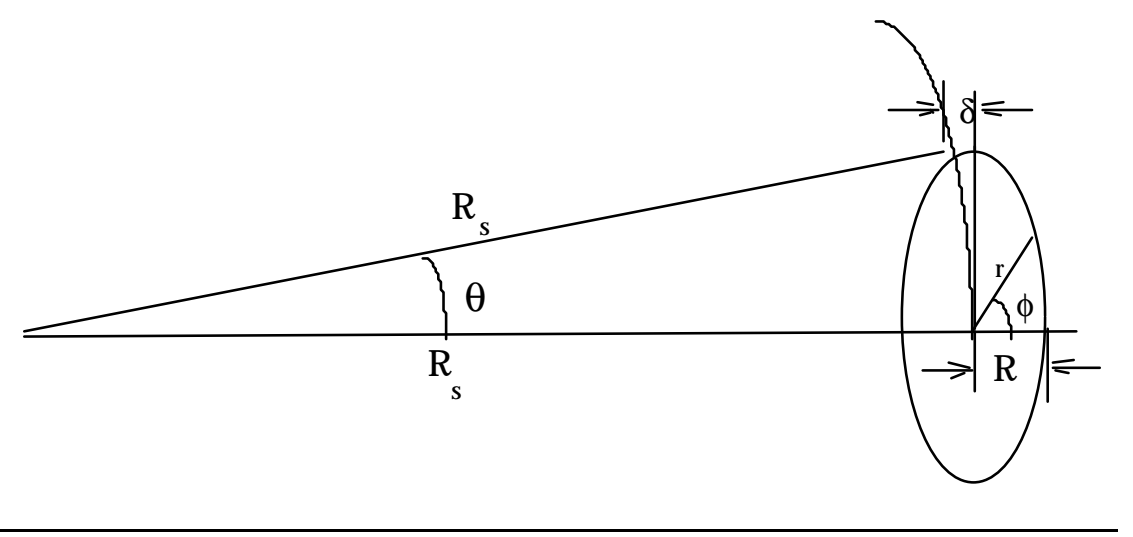

Figure 5: Simplified ray diagram for describing spherical errors. A ray is incident upon a circular aperture of radius $R$. The source distance is $R_{S}$, the error angle is $\theta$, the displacement error at the aperture is $\delta$, the polar coordinates of the aperture plane are $r, \phi$, and the radius of the aperture is R.

In the figure, $\delta=r \tan \theta \cong r \theta=r^{2} / R_{S}$, which gives $\delta=2 r^{2} / R_{S}$ over the entire aperture. If this is substituted in equation (2), we get, after Howells ${ }^{10}$

$$
\begin{aligned}
\mathrm{D}(\sigma) & =\frac{1}{\mathrm{~A}} \iint_{\mathrm{A}} \cos (2 \pi \sigma \delta) \mathrm{dud} \\
& =\frac{1}{\mathrm{~A}} \iint_{\mathrm{A}} \cos (2 \pi \sigma \delta) \mathrm{rdr} \mathrm{d} \phi \\
& =\frac{1}{\mathrm{~A}} \iint_{\mathrm{A}} \cos \left(2 \pi \sigma \frac{\mathrm{r}^{2}}{\mathrm{R}_{\mathrm{S}}}\right) \mathrm{r} \mathrm{dr} \phi \\
& =\frac{1}{\mathrm{~A}} \int_{0}^{\mathrm{R}} \cos \left(\frac{2 \pi \sigma}{\mathrm{R}_{\mathrm{S}}} \mathrm{r}^{2}\right) \mathrm{rdr}_{0}^{2 \pi} \mathrm{d} \phi \\
& =\frac{1}{\mathrm{~A}} \int_{0}^{\mathrm{R}} \frac{\mathrm{R}_{\mathrm{S}}}{4 \pi \sigma} \mathrm{d}\left[\left.\sin \left(\frac{2 \pi \sigma}{\mathrm{R}_{\mathrm{S}}} \mathrm{r}^{2}\right)\right|^{2 \pi}\right. \\
& =\left.\frac{\mathrm{R}_{\mathrm{S}}}{2 \sigma_{\mathrm{A}}} \sin \left(\frac{2 \pi \sigma}{\mathrm{R}_{\mathrm{S}}} \mathrm{r}^{2}\right)\right|_{\mathrm{r}=0} ^{\mathrm{r}=\mathrm{R}}
\end{aligned}
$$




$$
\begin{aligned}
& =\frac{\mathrm{R}_{\mathrm{S}}}{2 \sigma\left(\pi \mathrm{R}^{2}\right)} \sin \left(\frac{2 \pi \sigma}{\mathrm{R}_{\mathrm{S}}} \mathrm{R}^{2}\right) \\
& =\frac{\sin \left(\frac{2 \pi \sigma}{\mathrm{R}_{\mathrm{S}}} \mathrm{R}^{2}\right)}{\left(\frac{2 \pi \sigma}{\mathrm{R}_{\mathrm{S}}} \mathrm{R}^{2}\right)} \\
& =\sin \mathrm{c}\left(\frac{2 \sigma}{\mathrm{R}_{\mathrm{S}}} \mathrm{R}^{2}\right)
\end{aligned}
$$

where $\sin c x=\sin (\partial x) / \partial x$.

Satisfying our requirement that $D(\sigma)>0.9$ gives $\sigma R^{2} / R_{S}<0.25$, or

$$
\mathrm{R}_{\mathrm{S}}>4 \sigma \mathrm{R}^{2}=4\left(10^{6} \mathrm{~cm}^{-1}\right)\left(10^{-2} \mathrm{~cm}^{2}\right)=400 \mathrm{~m} .
$$

Now this radius of curvature pertains to a reflective surface perpendicular to the beam, so we have to adjust for the angle at which the beam strikes the mirrors and beamsplitters in determining their tolerances. Using the equation for calculating the focus of a beam striking a curved surface of radius $r$ we set

$R_{S}=(r \sin \theta) / 2$, giving for $R_{S}=400 m$

$$
\begin{array}{ll}
r>4.6 \mathrm{~km} & \theta=10^{\circ} \text { (mirrors) } \\
r>2.3 \mathrm{~km} & \theta=20^{\circ} \text { (beamsplitters) }
\end{array}
$$

\section{Tilt Errors}

As mentioned above, tilt errors, in which both beams are plane waves but are tilted with respect to one another, arise from poor optical surfaces, nonparallel mirrors, tilt of the mirrors with respect to the beamsplitters as a consequence of stage motion, and misalignment of the beamsplitters with respect to the mirrors.

It should be mentioned here, that of the three rotation errors possible in the optical elements in the system, roll, yaw, and pitch, the consequences of the first two on coherent recombination of the beams are considered negligible. Consider figure 3 . 
For small roll errors (i.e. around the z-axis) the mirrors are moving in their own planes; it would take a roll of $90^{\circ}$ for an error of $10^{\circ}$ to be introduced, since that is the incident angle of the beam to the mirror at zero roll. Rolling the beamsplitters would not introduce any tilt at all. For small yaw errors (i.e. around the vertical $x$ axis), the pair of mirrors for each separated beam act as a pentaprism, where the change in angle of one mirror is compensated by the same change in the next mirror because they are attached to each other. Yaw between the beamsplitters would introduce a shear.

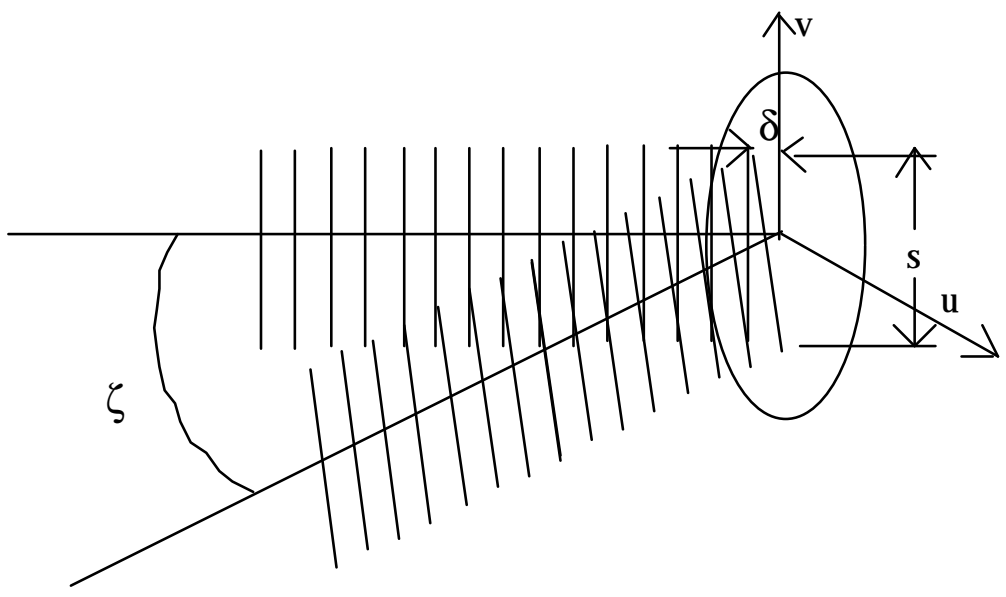

Figure 6: Ray diagram for tilt errors.

In this case, we have an error at the detector $\delta=\zeta v$ where $\zeta$ is the angle and $v$ the distance along the detector's Cartesian v-coordinate. Substituting this value for $\delta$ into equation 1 gives

$$
\begin{aligned}
\mathrm{D}(\sigma) & =\frac{1}{\mathrm{~A}} \iint_{\mathrm{A}} \cos (2 \pi \sigma \delta) \mathrm{du} d \mathrm{v} \\
= & \frac{1}{\mathrm{~A}} \iint_{\mathrm{A}} \cos (2 \pi \sigma \zeta \mathrm{v}) \mathrm{du} d \mathrm{v} \\
& =\frac{1}{\mathrm{~A}} \int_{-\mathrm{R}}^{-\mathrm{R}} \mathrm{dv} \int_{-\sqrt{\mathrm{R}^{2}-v^{2}}}^{\sqrt{\mathrm{R}^{2}-v^{2}}} \cos (2 \pi \sigma \zeta \mathrm{v}) \mathrm{du} \\
& =\frac{2 \mathrm{R}}{\mathrm{A}} \int_{-\mathrm{R}}^{-\mathrm{R}} \cdot \sqrt{1-\frac{\mathrm{v}^{2}}{\mathrm{R}^{2}}} \cos (2 \pi \sigma \zeta \mathrm{v}) \mathrm{dv}={ }_{2} \frac{\mathrm{J}_{1}(2 \pi \sigma \zeta \mathrm{R})}{(2 \pi \sigma \zeta \mathrm{R})}
\end{aligned}
$$


where the equation to the Bessel function on the last line may be found in one of the mathematics handbooks ${ }^{11}$. Now, $2 \frac{J_{1}(x)}{x}$ falls to 0.9 at $x=0.911$; for $R=1 \mathrm{~mm}$ and $\mathrm{s}=10^{6} \mathrm{~cm}$ we get $\zeta=1.5 \mu \mathrm{rad}$. A rule-of-thumb in interferometry is that light must recombine to within a quarter-wavelength for any reliable measurement to take place. (If the recombining waves are a half-wavelength off, we would get troughs combining with crests for no fringes at all.)

For a system aperture of $R=1 \mathrm{~mm}, \zeta R=1.5 \mathrm{~nm}<\lambda / 4=2.5 \mathrm{~nm}$, well within the rough tolerance. We can reasonably divide up the angular tolerance between the mirrors and beamsplitters by noting that making the beamsplitters was somewhat of a research and development project of its own; making flat mirrors and coating them is a well-understood technology. So the following tolerances were chosen:
Mirrors:
$0.5 \mu \mathrm{rad}$

Beamsplitters: $\quad 1.0 \mu \mathrm{rad}$ 
These error budgets hold for the surfaces of the manufactured components (their slope error), pitch of the stage thoughout its motion, and alignment of the beamsplitters. Since the beamsplitters can be aligned under computer control, tilts imparted to the two separated beams can be corrected while the stage is at rest by proper beamsplitter alignment. However, the beams will be shifted (sheared) with respect to each other. The beams must be within the coherence width to interfere. Coherence width is determined by the source size and distance from the source to the image, and is given by the equation ${ }^{12}$

$$
\begin{gathered}
\mathrm{w} \cong \frac{\lambda d}{\mathrm{R}} \\
\text { where } \mathrm{w}=\text { coherence width } \\
\lambda=\text { photon wavelength }=20 \mathrm{~nm} \\
\mathrm{R}=\text { source } \text { size } \cong 300 \mathrm{~mm} \\
\mathrm{~d}=\text { distance from source }=3 \mathrm{~m}
\end{gathered}
$$

When the values are substituted into the equation we get $\mathrm{w}=200 \mu \mathrm{m}$. This imposes an angular tolerance on the alignment of the mirrors with respect to each other, albeit a much looser one than that for the slope errors. Recombining the beams within their coherence width of $200 \mu \mathrm{m}$ requires that the mirrors be perpendicular and parallel within the angle $\mathrm{w} / \mathrm{L}$, where $\mathrm{w}$ is the coherence width and $L$ is the distance from the mirrors to the downstream beamsplitter $(\sim 1 \mathrm{~m})$. This gives an angular tolerance of $200 \mu \mathrm{rad}$ for parallelness and perpendicularity of the mirrors.

\section{Tilt error due to a reflecting surface}

Tilt errors are of prime importance in making an x-ray interferometer work, so a general discussion of their effects is in order. The following derivation follows Malcolm Howell's ALS Note on manufacturing tolerances for the x-ray mirrors, ${ }^{13}$ but is applicable to any tilt in the system. 
Consider figure 7. If the mirror is perfectly reflecting, the incident and reflected beams $\mathbf{r}_{\mathbf{i}}$ and $\mathbf{r}_{\mathbf{r}}$ have equal amplitudes (let's call them unit vectors in their respective directions) and angles $\theta$ with respect to the mirror. Thus the difference $\mathbf{r}_{\mathbf{i}}-\mathbf{r}_{\mathbf{r}}$ will be parallel to the normal unit vector $\hat{\mathbf{n}}$ and given by

$$
\begin{aligned}
\mathbf{r}_{\mathbf{i}}-\mathbf{r}_{\mathbf{r}}=2 \sin \theta|\mathbf{r}| \hat{\mathbf{n}} \\
=2\left(\mathbf{r}_{\mathbf{i}} \cdot \hat{\mathbf{n}}\right) \hat{\mathbf{n}}
\end{aligned}
$$

The reflected beam is

$$
\mathbf{r}_{\mathbf{r}}=\mathbf{r}_{\mathbf{i}}-2(\mathbf{r i} \cdot \hat{\mathbf{n}}) \hat{\mathbf{n}}
$$

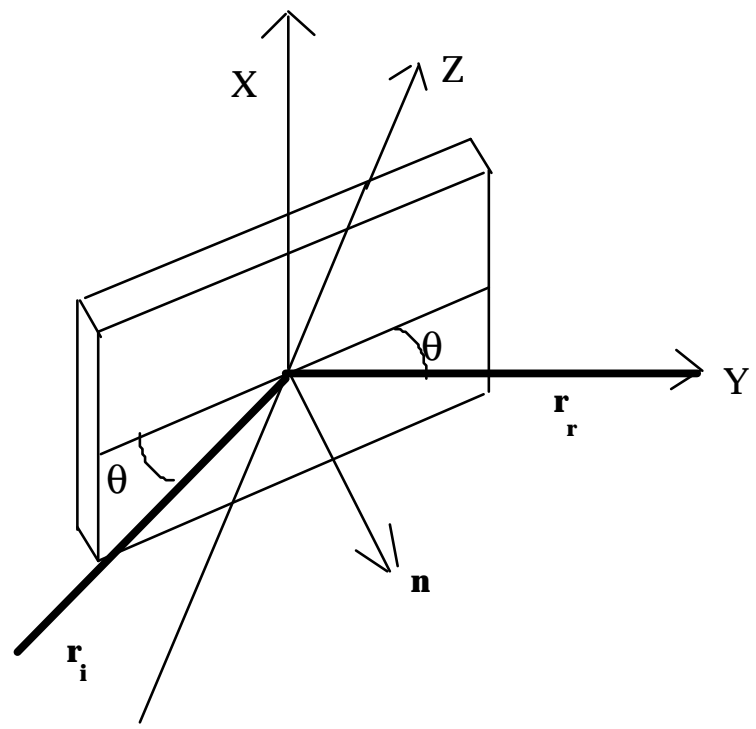

Figure 7: Schematic diagram for describing tilt errors for one of the x-ray mirrors. The laboratory reference frame is indicated, with the z-axis in the direction of stage motion, the $x$-axis vertical, and the $y$-axis orthogonal to them. In this case, the $y$-axis happens to be collinear with the reflected ray $r_{r}$, but this is not necessary to the general discussion. 


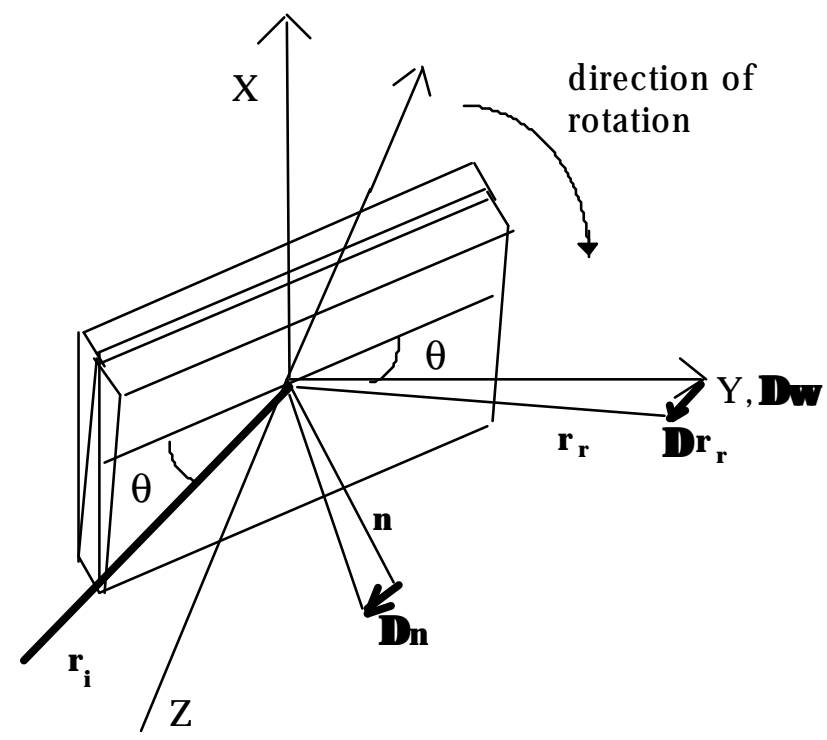

Figure 8: Schematic diagram for tilt errors. The mirror has been rotated.

If a rotation $\Delta \omega$ around the positive $\mathrm{Y}$-axis occurs (corresponding to a pitch error in the direction of stage motion), $\mathrm{n}$ will be rotated by a vector $\Delta \mathrm{n}$ given by

$$
\Delta \hat{\mathbf{n}}=\Delta \omega \times \hat{\mathbf{n}}
$$

So $r_{r}$ will correspondingly be rotated by a vector $\Delta r_{r}$ given by

$$
\begin{aligned}
& \Delta \mathbf{r}_{\mathbf{r}^{\prime}}=-2\left\{\left[\mathbf{r}_{\mathbf{i}^{*}}(\hat{\mathbf{n}}+\Delta \hat{\mathbf{n}})\right][\hat{\mathbf{n}}+\Delta \hat{\mathbf{n}}]-\left(\mathbf{r}_{\mathbf{i}^{*}} \hat{\mathbf{n}}\right) \hat{\mathbf{n}}\right\} \\
= & -2\left[\left(\mathbf{r}_{\mathbf{i}^{*}} \hat{\mathbf{n}}\right) \hat{\mathbf{n}}+\left(\mathbf{r}_{\mathbf{i}^{*}} \Delta \hat{\mathbf{n}}\right) \hat{\mathbf{n}}+\left(\mathbf{r}_{\mathbf{i}^{*}} \hat{\mathbf{n}}\right) \Delta \hat{\mathbf{n}}+\left(\mathbf{r}_{\mathbf{i}^{*}} \Delta \hat{\mathbf{n}}\right) \Delta \hat{\mathbf{n}}-\left(\mathbf{r}_{\mathbf{i}^{*}} \hat{\mathbf{n}}\right) \hat{\mathbf{n}}\right] \\
= & -2\left[\left(\mathbf{r}_{\mathbf{i}^{*}} \Delta \hat{\mathbf{n}}\right) \hat{\mathbf{n}}+\left(\mathbf{r}_{\mathbf{i}^{*}} \hat{\mathbf{n}}\right) \Delta \hat{\mathbf{n}}+\left(\mathbf{r}_{\mathbf{i}^{*}} \Delta \hat{\mathbf{n}}\right) \Delta \hat{\mathbf{n}}\right] \\
\cong & -2\left[\left(\mathbf{r}_{\mathbf{i}^{*}} \Delta \hat{\mathbf{n}}\right) \hat{\mathbf{n}}+\left(\mathbf{r}_{\mathbf{i}^{*}} \hat{\mathbf{n}}\right) \Delta \hat{\mathbf{n}}\right]
\end{aligned}
$$

The last step in the derivation comes from the approximation that $\Delta \hat{\mathbf{n}}$ « $\hat{\mathbf{n}} \Rightarrow$ $|\Delta \hat{\mathbf{n}}|^{2} \cong 0$. Substituting $\Delta \hat{\mathbf{n}}=\Delta \boldsymbol{\omega} \times \hat{\mathbf{n}}$ gives

$$
\Delta r_{r}=-2\left[\left(r_{i^{*}} \Delta \omega \times \hat{n}\right) \hat{n}+\left(r_{i^{*}} \hat{n}\right)(\Delta \omega \times \hat{n})\right]
$$

Now, the scalar product $\mathbf{r}_{\mathbf{r}} \cdot \Delta \mathbf{r}_{\mathbf{r}}=0$, which means the two vectors are perpendicular. Since $\left|r_{r}\right|=1$ ( $r_{r}$ is a unit vector), the tangent of the angle $\alpha$ rotated by $r_{r}$ will be 


$$
\tan \alpha=\frac{\left|\Delta \mathbf{r}_{\mathbf{r}}\right|}{\left|\mathbf{r}_{\mathbf{r}}\right|}=\left|\Delta \mathbf{r}_{\mathbf{r}}\right| \cong \alpha
$$

where the last step comes from the small-angle approximation. So the magnitude of the rotation vector is actually the angle by which the reflected beam changes when the mirror is rotated by $\Delta \boldsymbol{\omega}$. If $\Delta \boldsymbol{\omega}$ is around the $y$-axis (pitch error)

$$
\begin{aligned}
\Delta \mathbf{r}_{\mathbf{r}} & =-2\left[\mathbf{r}_{\mathbf{i}} \cdot(|\boldsymbol{\Delta} \boldsymbol{\omega}||\hat{\mathbf{n}}| \cos \theta \hat{\mathbf{x}}) \hat{\mathbf{n}}+\left(\left|\mathbf{r}_{\mathbf{i}}\right||\hat{\mathbf{n}}| \sin \theta\right)(|\boldsymbol{\Delta} \boldsymbol{\omega}||\hat{\mathbf{n}}| \cos \theta \hat{\mathbf{x}})\right] \\
& =-2 \cos \theta|\boldsymbol{\Delta} \boldsymbol{\omega}| \sin \theta \hat{\mathbf{x}}) .
\end{aligned}
$$

The first term disappeared since $\mathbf{r}_{\mathbf{i}} \perp \hat{\mathbf{x}}$. Also $\mathbf{r}_{\mathbf{i}}$ and $\hat{\mathbf{n}}$ are unit vectors. The ratio of the magnitudes will tell us how much the reflected beam moved for a given pitch error $\Delta \omega$ :

$$
\frac{\left|\Delta \mathbf{r}_{r}\right|}{|\Delta \boldsymbol{\omega}|}=2 \cos \theta \sin \theta=0.342 \text { for } \theta=10^{\circ}
$$

The mirrors move as a unit; their geometry dictates that a pitch error will be quadrupled, since one set of mirrors will reflect the beam up twice, and the other set will reflect it down twice by the same amount. The tilt error was estimated above to be $1.5 \mu \mathrm{rad}$, so the maximum allowable pitch error for the stage travel (in a system that is perfectly aligned while the stage is at rest) is

$$
|\boldsymbol{\Delta} \boldsymbol{\omega}| \frac{\left|\Delta \mathbf{r}_{\mathbf{r}}\right|}{8 \cos \theta \sin \theta}=\frac{1.5 \times 10^{-6}}{8 \cos 10^{\circ} \sin 10^{\circ}}=1.1 \mu \mathrm{rad}
$$




\section{Description of Mach-Zehnder interferometer}

\section{Mechanical drive}

\section{Flexure Mechanism}

The very high resolution obtainable from an interferometer is inversely proportional to the path length difference introduced between the separated beams of light. In this case, we must introduce a path length difference that is a million times the wavelength of the light, while continually recombining the beams coherently (i.e. with constant phase). This places two demands on the mechanical system used to introduce the PLD: it cannot introduce excessive tilt or indexing (position) errors. As mentioned above, the long distance requires the use of a flexure mechanism

A flexure hinge is used in applications where the stick-slip caused by two surfaces sliding along each other, as in conventional hinges, is unacceptable. It consists of a thin piece of material, usually metal, that may be bent many times without fatiguing. It is much longer along the axis of rotation to prevent rotation around other axes and to provide mechanical stability. The shape of a flexure hinge and the material from which it is made determine its mechanical properties, the important ones being maximum angle of rotation, displacement of the hinge point (center shift), and fatigue due to stresses within the material and number of rotations. Three possible configurations for flexure hinges will be compared here: flat, crossed-strip, and cartwheel. $^{14}$

A simple flexure hinge may be constructed out of a thin, flat piece of spring steel. Fabrication of a flat hinge is easy, and since the stress to the hinge is distributed over its length, it has low fatigue. However, as a flat hinge bends, the hinge point moves, not necessarily predictably. 


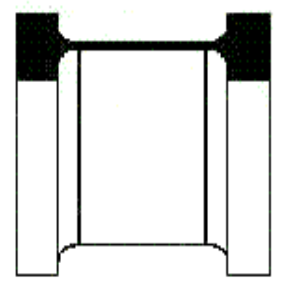

A

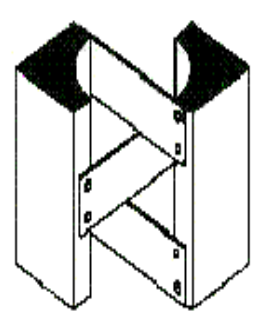

B

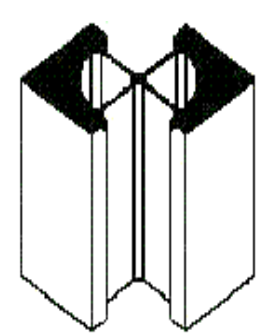

c

Figure 9: Flexure types. A: A strip hinge is fairly easy to make, but it has a large center shift. Also, there are no strips to prevent the two ends from twisting around an axis connecting them. B: A crossed-strip hinge prevents twisting by constraining that rotation with a strip at right angles to it. However, they cannot be produced along with the rest of the flexure mechanism. C: Cartwheel flexures can be produced with EDM techniques along with the rest of the mechanism.

Crossed-strip hinges actually produce a worse center shift than a flat hinge (by a factor of $\sqrt{2}) \cdot{ }^{15}$ However, theoretical calculations demonstrate that a monolithic cartwheel hinge reduces the center shift by a factor of 3.6. ${ }^{16}$ Also, crossed-strip hinges cannot be produced with electric discharge machining (EDM) as part of a monolithic flexure mechanism. Thus a cartwheel-type flexure hinge was selected (see figures 9 and 10).

The hinges, as well as the test of the flexure mechanism, were monolithically cut from a 14" $\times 10$ " $\times 2$ " piece of maraging steel. The choice of this very hard, lowfatigue steel was relatively expensive, but proved necessary when we had to modify the flexure mechanism later to correct for manufacturing flaws. 


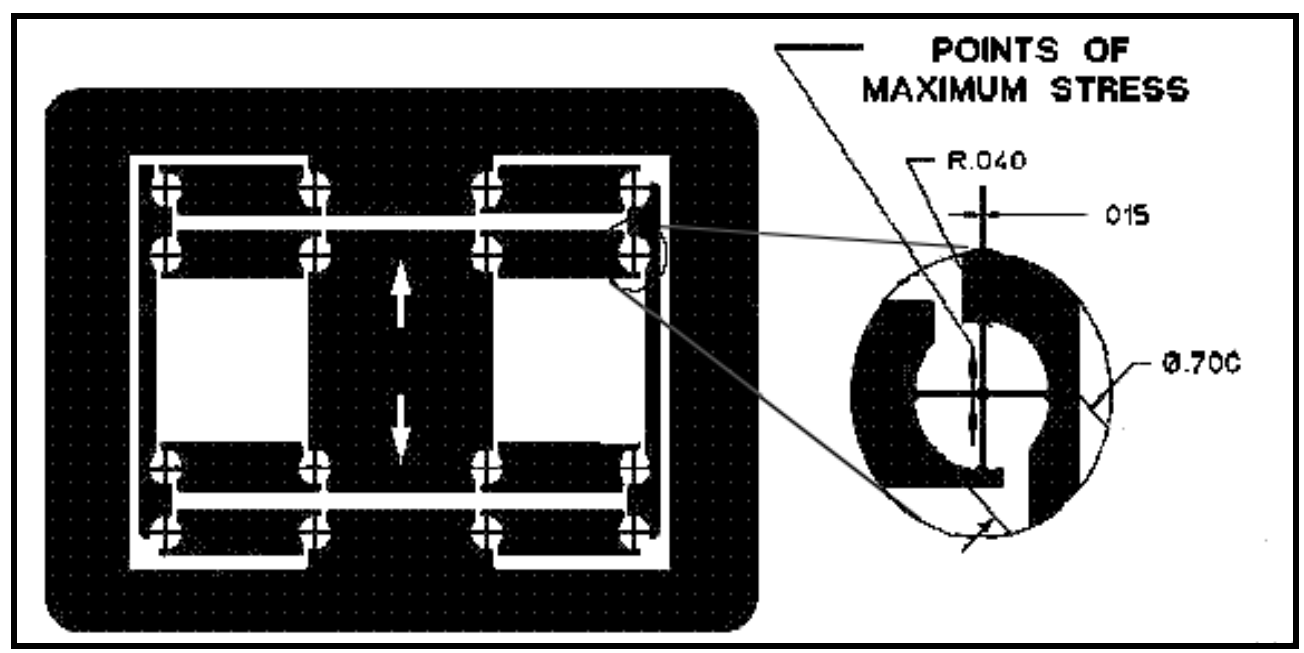

Figure 10: Schematic of the flexure mechanism. The stage at center is shown in the center of its traversal range, at zero path length difference. Comparing this figure to figure $\mathbf{1 5}$ gives an idea of how the mechanism deforms when the stage is driven to one end. The points of maximum stress indicated in the magnified diagram of one of the flexures occur just where the hub at the center of the hinge tapers to the width of the spoke. This design for the flexure mechanism was chosen to halve the angle that each hinge rotates, thus greatly reducing the stress on the hinge.

The design shown in figure 10 was cut using electric discharge machining (EDM), a process in which, after a rough cut is made, a wire at $\sim 500 \mathrm{~V}$ is brought near the surface to be machined by a numerically controlled arm. The wire is tightly stretched into a straight line by its mounting, and moved parallel to the surface by the arm.

Any protuberance above the surface is ablated by the resulting spark discharge; this material is deposited on the passing wire. The wire is unrolling from a large spool, so that the "plated" wire moves away from the next section of unmachined steel. The result is that a very flat, smooth surface is produced that follows the contour of the arm's path.

\section{Driver}

A large hydraulic driver was custom-designed for the interferometer at LBNL (see figure 11). Of primary consideration in its design, besides producing enough force to move the stage and mirrors through their entire range, was minimizing stick-slip. Using a continuous-motion driver with fast, precise position measurement electronics instead of a stepper motor eliminated problems associated with motors 
and gears such as backlash and lead-screw wobble. Errors introduced by these mechanisms are difficult enough when indexing position at the micron level. They become nearly intractable at the nanometer level. Piezoelectric materials can provide repeatable Angstrom-resolution motion, but have a limited range of travel. The problem of stick-slip will be discussed in detail below, but briefly, it is measured as a nearly discontinuous "jump" in position while moving the stage at a constant velocity.

The housing was machined from a solid piece of brass, 8.000" $\times 4.000 "$ x 4.000", with a bore diameter of 2.500". The piston was also machined from brass and has dimensions 2.497" OD and 1.003" ID to accommodate the bore of the housing and the driveshaft, respectively. It has a 0.184 " deep gland (notch for an O-ring) cut in OD and a 0.184 " deep gland cut in ID. The piston is fastened to the driveshaft with a tapered brass collet. The collet fits into a tapered hole in the piston and both have matching threaded holes for mating them; screwing them together forces the tapered collet against the driveshaft.

The driveshaft has several parts to it: a hollow tube, 1.000" OD and 0.750" ID x 17" that has a 2.5" conflat flange welded to it on one end for bolting it to the vacuum system via a bellows, an aluminum connecting rod, $0.625 " \times 24$ " that has a 1/4-20 threaded hole at either end to accommodate the flexure joints, two stainless steel flexure joints, $0.625 " \times 4.05$ " that have a machined neck, 0.080 ", allowing for small angular errors in alignment of the axis of the driveshaft and the center point of the stage, a taper pin, 0.625 " at the large end tapering to 0.500 " at the small end for wedging the collet between the taper pin and the ID of the hollow tube, and a collet, a piece made from a stainless-steel tube by cutting slits from one end of the tube to within $1 / 4$ " of the other end, alternated with similar ones cut from the other end, forming a sort of zigzag tube. Screwing in on the taper pin forces the collet against the ID of the driveshaft, locking the connecting rod to the driveshaft. 
Figure 11: Schematic of the driver. The piston, housing, and end caps are all made out of brass. A polished stainless steel hollow driveshaft runs through the center of the driver; this is the sliding surface when the piston moves. The driveshaft slides against O-rings at either end of the housing, and is wetted with oil on both sides of the O-ring. Inside the driveshaft, a connecting rod is fastened to one end with a collet; the other end is free. A machined flexure joint between the connecting rod and the collet allows the connecting rod to move about freely within the driveshaft. The other end of the connecting rod is fastened to the flexure stage with another flexure joint, thus allowing small angular movement by the connecting rod without putting a large torque on the stage.

We chose mineral oil as the hydraulic fluid; there are commercial hydraulic fluids that have better frictional and viscosity characteristics, but mineral oil is readily available and is good for the skin. The direction and speed of the piston was controlled by a system of valves, pressure regulator, and Tygon ${ }^{\circledR}$ tubing connected to ports on either side of the piston. We found the Tygon tubing to be too elastic; the piston would keep moving after the pressure was released. In the final version 
of the hydraulic system the Tygon was replaced with rigid copper tubing. Also in the final version we added solenoid-actuated valves so that spectra could be acquired completely under computer control.

\section{X-ray Optics}

The optics of the interferometer consist of four mirrors and two beamsplitters.

\section{Beamsplitters}

Two types of beamsplitters exist: amplitude-dividing and wavefront-dividing. An example of an amplitude-dividing beamsplitter is the half-silvered mirror used in the Michelson-Morley experiment to demonstrate the absence of a light wave propagating ether. The beam must be transmitted through some material such as glass and either be reflected along one leg of the interferometer or transmitted along another. However, soft x-rays are quickly absorbed for most materials, so we selected a wavefront-dividing beamsplitter.

A wavefront-dividing beamsplitter separates a plane wave into a relatively small number of waves. It consists of a number of alternating slits and flat, mirrored tines that form a grating. One may get a sense of scale from figure 12. The second drawing shows a cutaway side view of the beamsplitter. Light would enter from below at an angle of $20^{\circ}$ with respect to the plane of the beamsplitter. Half of the light would be transmitted through the slits and half would be reflected by the tines. The rear of the beamsplitter is cut away at a $10^{\circ}$ angle to prevent any blocking of the transmitted beam. 
This EPS image does not contain a screen preview.

It will print correctly to a PostScript printer.

File Name : bs_1.epsi

Figure 12: Beamsplitter schematic.

Four beamsplitters were each made from a single crystal of silicon by Boeing North American. Each beamsplitter is a rectangular block of pure silicon, $95.52 \mathrm{~mm} x$ $24.00 \mathrm{~mm} \times 5.00 \mathrm{~mm}$ (approx. $33 / 4 " \times 1 " \times 1 / 5 ")$. (See figure 12.) The reflecting surface was ground and polished to a roughness of $<5 \AA \mathrm{rms}$ and a slope error of $<0.75 \mu \mathrm{rad} \mathrm{rms}$. The $100 \mathrm{~mm}$ period slots were made by using a photolithographic mask and then anisotropically etching the silicon along the (110) crystalline plane using $\mathrm{KOH}$ as the etchant (see figure 13). Finally, the beamsplitters were coated with $150 \AA$ of molybdenum using vapor deposition, a process that produces extremely smooth surfaces. ${ }^{17}$ Molybdenum has one of the highest reflectivities for $x$-rays of any known material. ${ }^{18}$ 


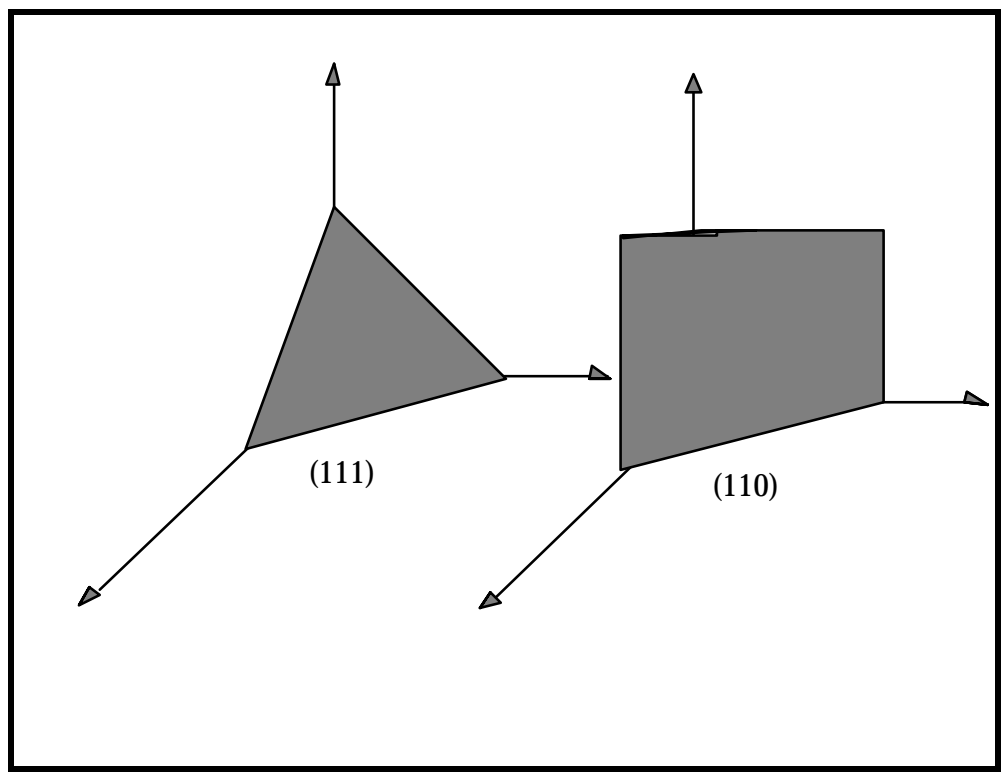

Figure 13: Two of the many etch planes. (111) is not etched, while (110) is etched. Roughly, the longer the interatomic bonds, the weaker the interatomic forces, and the easier it is to break them with an etchant.

After getting the beamsplitters from Boeing, we installed them and began testing and aligning them manually. However, unknown to us at the time was the fact that molybdenum oxidizes fairly quickly, in less than a month. The beamsplitters were open to air for a substantially longer time. The oxidation that took place made the beamsplitters rough and required our stripping the molybdenum off with $3 \%$ hydrogen peroxide and having them recoated. They were quickly reinstalled and the vacuum system was pumped down to $10^{-5}$ torr This worked. Following are the theoretical tolerances and their measured values:

\begin{tabular}{|l|l|l|}
\hline Type of error & Theoretical & Measured \\
\hline \hline Slope error & $<1.0 \mu \mathrm{rad}$ & $<0.7 \mu \mathrm{rad}$ \\
\hline Roughness & don't know & $<3.5 \AA$ \\
\hline spherical error radius & $>2.3 \mathrm{~km}$ & $>6.4 \mathrm{~km}$ \\
\hline
\end{tabular}


Mirror Assembly

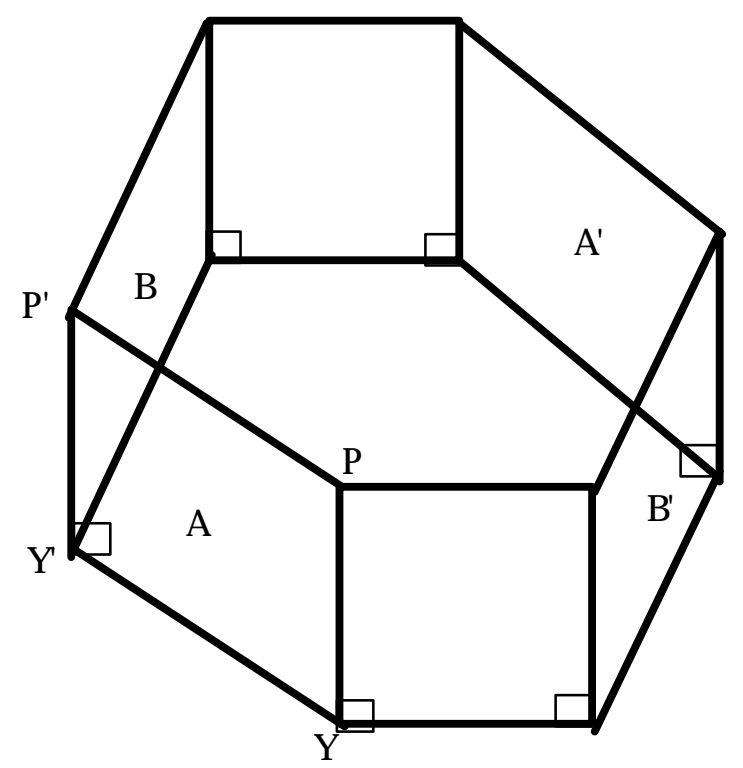

Figure 14: Schematic of the prism used to mount the $x$-ray mirrors. The vertices of adjacent faces that need to be perpendicular are indicated with the right-angle symbol $L$. Thus the faces labeled A, A', $B$, and $B^{\prime}$ are all perpendicular to the base. They are also the faces to which the four $x$-ray mirrors are optically contacted. In addition, opposite faces of the prism must be parallel to each other, so A || A' and B || B'. The face labeled PYY'P' corresponds to the end-on view of side PY illustrated in figure 16, in which the apparatus for measuring the parallelness and perpendicularity of the prism is discussed.

The mirror assembly was made from five separate pieces: four identical rectangular blocks of glass and a diamond-shaped glass prism. The upper half of one face of the blocks was initially coated with $500 \AA$ of molybdenum to give them a mirror finish, but this was later stripped off due to oxidation. The mirrors were then mounted on the prism by carefully sticking them on its perimeter. No adhesive is necessary (or desired) if the matching surfaces are flat and smooth enough; The electrostatic contact forces are strong enough to hold the assembly together. 
Photon Sciences, the manufacturer of the mirror assembly, qualified it by using several optical instruments: parallelness and perpendicularity were measured using an Haidinger fringe test, described below; surface roughness was measured with a WYKO TOPO 2D surface profiler; slope error was estimated with a WYKO 6000 interferometer and $\lambda / 30$ test plates.

The Haidinger fringe test exploits the movement of Haidinger fringes to measure the error in perpendicularity and parallelness of the prism. Haidinger fringes appear as rings or lines between two nearly parallel surfaces when monochromatic, coherent light (a laser) is shined normal to the surfaces. The surfaces may bound any medium (e.g. they may be opposite sides of a block of glass or the faces of two plates of glass with air between the faces). The fringes arise, of course, from incident light interfering with light reflected from the downstream surface. The interference pattern appears at the upstream surface and the image has its focus at infinity.

If either the observer or the test sample is scanned relative to the other, the fringes will move; our eyes are sensitive to motion and it is this procedure that is used in calibrating the prism. One fringe of motion corresponds to an error of $\lambda / 4$. (The conditions for maxima and minima are:

$$
\begin{array}{lll}
d=(m / 2+1 / 4) \lambda / n & m=0,1,2, \ldots & \text { maxima } \\
d=m \lambda / 2 n & m=0,1,2, \ldots & \text { minima }
\end{array}
$$

where $d$ is the distance moved, $m$ is the order index of diffracted light, $\lambda$ is the wavelength of light, and $\mathrm{n}$ is the index of refraction of the glass. These equations include the $180^{\circ}$ phase shift when $n_{2}>n_{1}$ and $0^{\circ}$ phase shift when $n_{2}<n_{1} . \quad\left(n_{1}\right.$ and $n_{2}$ refer to the indices of refraction of the media in which the light is incident and refracted from the interface, respectively. The two media are air and glass.) If we plug in the same $m$ in both equations we get $d=\lambda / 4$. Glass also amplifies the sensitivity of the test by shortening the light's wavelength by 1.5 , the index of refraction, and a trained observer can detect motion of 0.1 fringe. Putting all this together, the minimum observable positional error is $\lambda / 40=633 \mathrm{~nm} /\left(1.5^{*} 40\right)=11$ $\mathrm{nm}$. 


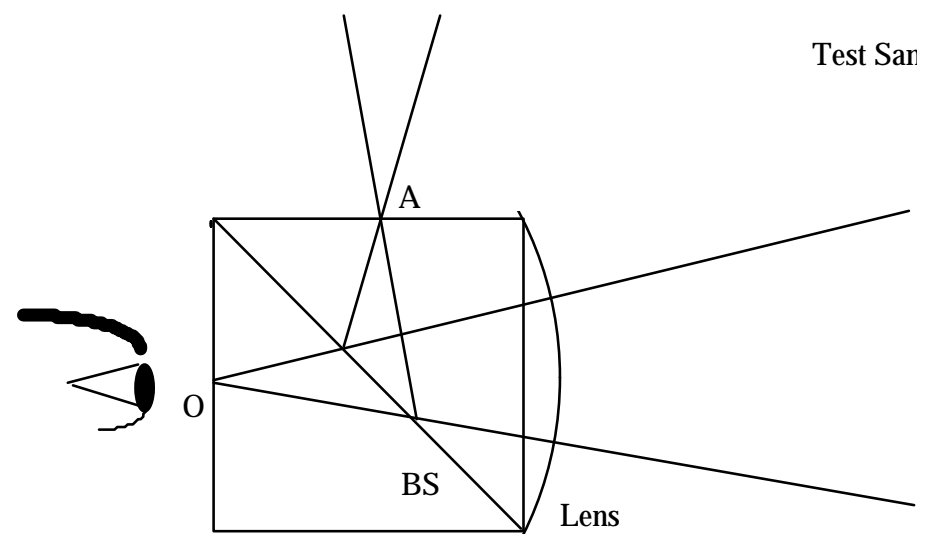

Figure 15: The Shack interferometer. Light enters at A, is reflected off the beamsplitter BS and is reflected either internally from the lens or externally from the test sample. The light beams returning from these two interfaces interfere and produce fringes at 0 .

Referring to figure 16, test prism LMN is placed atop of prism PYRS. $X Y Z$ is (should be) a $45^{\circ}$ right triangle. The perpendicular bisector of hypotenuse $X Z$ should bisect $\angle Y$, forming the $45^{\circ}$ right triangles $Z O Y$ and $Y O X$. The test beam enters the test prism at $A$ and follows the path A-B-C-D-C-B-A.

The fringe pattern is created between the two surfaces $X O$ and $O Z$ via the roof angle $X Y Z$. Thus, as $X O Z$ is a continuous surface, lack of any motion in the fringe pattern verifies that angle $X Y Z$ is indeed $90^{\circ}$. In considering whether the angle is smaller or larger than $90^{\circ}$, it should be noted that the test becomes a measure of the equality of $X Z$ relative to $2 Y O$-- the height of the prism -- and $X Z$ is greater than or less than $2 Y O$ corresponding to the angle being greater than or less than a right angle. 


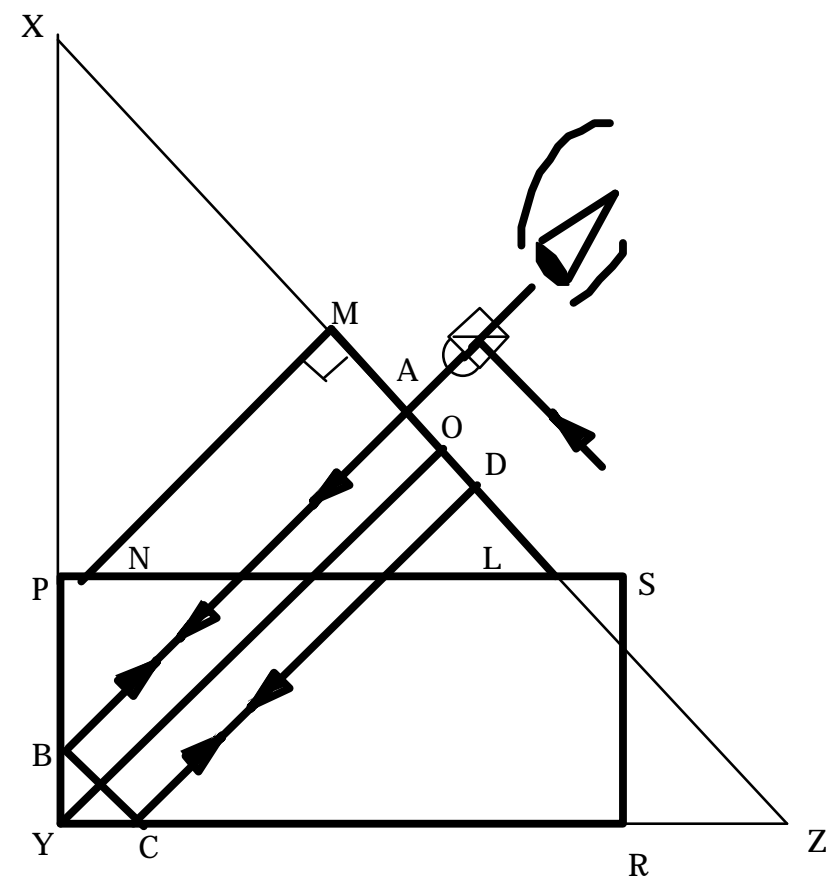

Figure 16: Test setup for determining perpendicularity of a side of the mirror prism (PY) to its bottom (YR). (This diagram should be regarded as an idealized end-on view of one of the sides of the diamond-shaped prism. The rest of the prism has been deleted for the sake of clarity.) Light from the Shack interferometer enters the test prism LMN at $A$ and follows the path $A B C D$, is reflected at $D$, and returns along that path. The test beam is scanned from $M$ to $O$; if the angle $Y$ is not $90^{\circ}$, the beam will not return to $A$ but a distance from $A$ proportional to the error in angle $Y$ and path length $2 A B C D$. The Haidinger fringes observed at the Shack interferometer will thus move across the field of view.

Consider the prism $X Y Z$. The dimensions $X O, O Z$, and OY are approx. $70 \mathrm{~mm}$. Assume that, in scanning the Haidinger fringes from $X$ to $O$, there is an error of one fringe (316.5 $\mathrm{nm}$ for $\mathrm{HeNe}$ ). This implies that $2 \mathrm{XZ}$ is one fringe different from $4 \mathrm{OY}$. Actually, the dimensional difference is $\sim 211 \mathrm{~nm}$ due to the index of the glass.

Therefore,

$$
\begin{aligned}
2 X Z & =4 X O=4 O Y+211 n m \\
O & =O Y+53 n m
\end{aligned}
$$

Now,

$$
\begin{aligned}
\tan (\angle X Y O) & =X O /(O Y+53 \mathrm{~nm}) \\
& =70 \mathrm{~mm} / 70.000053 \mathrm{~mm} \\
& =0.99999924286
\end{aligned}
$$




$$
=1-\left(0.757 \times 10^{-6}\right)
$$

or, since $\tan (\angle \mathrm{XYO}) \quad \angle \mathrm{XYO}$ for small angles, the error is $0.757 \mu \mathrm{rad}$. For the $90^{\circ}$ angle $X Y Z$, the error is just twice this, or $1.514 \mu$ rad per fringe over the scan length $X O$. However, the actual scan distance is only from $M$ to $O$, about $1 / 4$ of $X O$. So $1 / 4$ fringe over this length gives the $1.5 \mu$ rad error in $90^{\circ}$. Our specification is 2.5 $\mu \mathrm{rad}$, or about 0.4 fringe. This tolerance is actually much less than the $200 \mu \mathrm{m}$ tolerance for shear derived in the optical tolerances section above (p. 17). The engineers who use this method state "In reality, it is quite easy to observe and fabricate to $1 / 10$ fringe" 19 .

Following are the theoretical tolerances of the mirror assembly and their measured values:

\begin{tabular}{|l|l|l|l|}
\hline $\begin{array}{l}\text { Optical } \\
\text { component }\end{array}$ & Type of error & Theoretical & Measured \\
\hline \hline Mirrors & Slope error & $0.5 \mu \mathrm{rad}$ & $0.5 \mu \mathrm{rad}$ \\
\hline & Roughness & $<4 \AA$ & $1.7 \AA$ \\
\hline & Spherical error radius & $>4.6 \mathrm{~km}$ & $>6.0 \mathrm{~km}$ \\
\hline Prism & perpendicularity & $\pm 2.5 \mu \mathrm{rad}$ & $<0.6 \mu \mathrm{rad}$ \\
\hline & parallelness & $\pm 2.5 \mu \mathrm{rad}$ & $0.6 \mu \mathrm{rad}$ \\
\hline
\end{tabular}

\section{Data acquisition}

\section{Hardware}

\section{Measurement of stage position using HP laser interferometer}

Position of the stage can be measured to $\sim 3 \AA$ with the use of a heterodyning laser interferometer system from Hewlett--Packard. The system consists of:

5517B Dual-polarization Dual-frequency Helium-Neon laser head \& power supply

10897B Laser Axis Board, 6U VME configuration

10716A High-resolution Interferometer

10780F Remote Receiver, with fiber optic lens connection 
We provided the VME crate and controller, and another member of our team (Eddie Moler) wrote the driver software for the laser positioning system. In addition, we provided ancillary components: mirror and mirror mount for the stage, mirror and mirror mount to angle the laser light into the viewport on the side of the vacuum chamber in which the x-ray interferometer resides, mount for the laser head, and a mount for the laser interferometer.

\section{Description of HP laser interferometer}

The HP laser interferometer measures position to a resolution of $\sim 3 \AA$ in the following way: ${ }^{20}$

a. Generate a dual-polarization, dual-frequency laser beam by imposing an axial magnetic field on the helium-neon gas mixture before excitation. This splits the energy levels of the light-emitting electrons, and confers opposite circular polarization on the two slightly different frequencies of emitted light. The engineers then used waveplates to convert the circularly polarized components into orthogonal linearly polarized beams.

b. Sample part of the dual-frequency beam within the laser head to create a reference frequency.

c. Send the rest of the beam to the interferometer, where a polarizing beamsplitter separates the two frequencies (which are also orthogonally polarized) and sends them along different paths. One beam hits a retroreflector inside of the interferometer to provide a non-moving reference beam. The other beam goes to the mirror on the thing you want to measure. In the high-resolution interferometer, the measurement beam is reflected four times from the measurement mirror. Since the path-length difference of the moving and nonmoving beams are doubled for each pass (once going to the mirror and once coming back), four bounces multiplies the path-length difference by eight.

d. Recombine the two beams inside of the interferometer and send them to the receiver, whose detector senses the beat frequency and converts it into a train of pulses at that frequency. 
e. Send the reference and measure frequencies on to the Laser Axis Board. Here the reference waveform is electronically subdivided into 256 equal timeslices. The edge of the (rectangular) measurement waveform is then binned onto one of the 256 timeslices, thus improving the resolution by 256 . This improvement, taken together with the eightfold improvement due to the multiple passes of the measurement beam, give a resolution enhancement of 2048. The wavelength of HeNe light is 633 $\mathrm{nm}$, so the resolution is $633 \mathrm{~nm} / 2048=0.309 \mathrm{~nm}$.

The position is sampled at a rate of $10 \mathrm{MHz}$. HP guarantees that the latest datum in the position register is no older than $0.290 \mu \mathrm{sec}$.

\section{Alignment of HP laser interferometer}

We mounted the measurement mirror beneath the stage, collinear with the driveshaft axis. The laser was mounted on a tripod made out of aluminum plate, threaded rod, nuts, and washers (designed and manufactured by grad students). We found it necessary to mount another mirror on a magnetic stand in order to angle the laser light into a viewport on the side of the vacuum chamber. Alignment then consists of getting the laser beam to go in the top port of the interferometer and come out the bottom port without hitting the sides. The receiver has a green LED on it that lights up when it gets a beat frequency. If the alignment is slightly off, apparently only two of the four passes are traversed by the measurement beam; the distance measured is half of what it should be. Alignment must be experienced to be understood.

\section{Measurement of $x$-ray signal intensity}

The $x$-ray signal is measured in one of two ways: a silicon photodiode or a gas cell filled with helium under low pressure. The former method measures electrons liberated from their valence bands by incoming x-rays. The number of electrons, and therefore the measured current, are proportional to the number of photons and their energies. Theoretically, the number of electrons liberated is proportional to an integral multiple of the band gap in silicon, about $3.61 \mathrm{eV}$. So for $64 \mathrm{eV}$ photons, 
we would expect 17 electrons and holes to be liberated. (The actual response of the photodiode depends on a number of things, such as the thickness of any oxide layer formed on the surface of the diode and impurities. ${ }^{21,22}$ ) We used a Hamamatsu silicon PIN photodiode, model S3580-19.

The photodiode current was amplified and transduced into a voltage with a Keithley 428 Current Amplifier. The output of the amplifier was fed into an analog-to-digital converter (ADC) board.

Two VME boards, manufactured by Spectrum Communications, are used in acquisition of the x-ray signals: a carrier board (CV2) for the digital signal processor (dsp) and a Daughter Module Carrier Board (DMCB) for the analog-todigital converter. They are linked externally via the VMEbus backplane and internally by a 32-bit flat ribbon cable (the dBEX32 bus).

The CV2 provides interfacing between the VMEbus (i.e. the outside world--us), and the DSP and DMCB. It has shared memory that is available to the VMEbus and the module that the DSP is mounted on. This module (called TIM after Texas Instruments Module) has its own local memory and TI's TMS30C40 DSP. Interfacing to the VMEbus is provided by the VMEbus Interface Control/VMEbus Address Control (VIC/VAC) chip.

The DMCB can carry up to four modules of various functions. We use one 16-bit ADC module. The DMCB interfaces its modules with the dBEX32 via AMELIA2 chips, application-specific integrated circuits designed by Spectrum Communications. The ADC is Burr-Brown's AM/D16SA, a $200 \mathrm{kHz}$ 16-bit resolution $A D C$.

\section{Software}

Software for the data acquisition system was constructed at three levels: the lowest level was a program written in $\mathrm{C}$ and assembly for the dsp; the middle level was a $\mathrm{C}$ program written for the VMEbus controller that connected the dsp to the Sun workstation; the top level consisted of a set of LabVIEW panels that controlled experimental variables and displayed the data. The programs and program 
environments will be briefly described here; complete descriptions of the dsp and VxServer programs are included in Appendix 2.

\section{Digital signal processor (DSP) program}

The dsp program, written in $\mathrm{C}$ and assembly, acquires data from three data channels (viz. position and two x-ray intensity signals), and sends them to the VME controller as one of several streams: raw or filtered, binned or unbinned, or normalized signal data. Raw data means a time series capture of signal or position. Filtered data are raw data processed by the dsp through a finite impulse response (FIR) algorithm to eliminate noise. Binned data can be represented as a signal vs. position plot; although they are acquired separately, the x-ray signal depends upon the stage position. We can pick a position bin size and average all the $x$-ray signals that fall into that bin. Therefore it is possible to obtain a higher position resolution than from a single measurement. Normalized signal data are data from the position-varying $x$-ray channel divided by the data from the reference $x$-ray channel. This is done to eliminate changes in the x-ray signal external to the experimental setup, such as flux variations or motion of the beam on the detector.

\section{vxWorks}

vxWorks is a real-time Unix-based operating system. While most of the shell commands are identical to those in Unix , the kernel is considerably more compact, and there are some additions useful to a real-time system, such as a command line interpreter of most $\mathrm{C}$ instructions and the capability to change the values of global variables while the program is running.

The server program evolved from a continually running program that polled flags from the dsp and user interface, to one that initializes interrupts and semaphores, and then blocks until it receives one of these signals. The strategy is to make the CPU wait until something needs to be done, like transfer data, and then do it immediately. In the earlier version, the CPU continually cycled through an infinite loop while polling flags; this means that an important task might have to wait while the CPU went through a whole cycle. 


\section{VxServer Program Description}

In a similar fashion to the dsp program, I will describe the behavior of only one datastream, "raw signal 1 binned by raw HP position." The main purpose of this program is to serve as an interface between the dsp program and the user interface. The user sends a data request from the LabVIEW user interface (running on a Sun workstation) to VxServer (running on the MVME167 controller, hereinafter referred to as the 167). Since the two programs reside on different computers, the request is sent via a Remote Procedure Call (RPC). The source code for this highlevel protocol can be automatically generated by using a Unix function "rpcgen." It is not described here more than by stating that the RPC task server on the local machine blocks CPU execution of local functions until it receives a properly constructed command sent from a remote computer.

Once the RPC for data acquisition is received by s1hpctrl_1, the mirror stage is moved to the start position, the pertinent parameters for this data stream are set to their values, and the S1HP flag in the channel_go register is set. The dsp continuously polls this register, and when it sees the flag immediately starts collecting data for that data stream.

The dsp sends an interrupt to VxServer when one of its data arrays is filled. The applicable interrupt handler gives a semaphore (software "interrupt") to other code that is blocking CPU execution until the semaphore is taken. That code in turn does whatever processing is necessary and forwards the data array to the user interface on the Sun workstation.

\section{LabVIEW}

LabVIEW is a graphically-based language designed for realtime data acquisition, processing, and control. The programmer designs a front panel containing controls 
(e.g. start and stop buttons, numerical and text string entry boxes, and pull-down menus) and indicators (e.g. "LEDs", numerical and text string outputs, and 2D and $3 \mathrm{D}$ graphs, images, and even pictures of incoming data). The guts of the interactions of the front panel elements are contained in the block diagram associated with the front panel. Each front panel element created also has an icon in the block diagram; these block diagram icons may then be "wired" together using a colored line created by clicking on one icon with a mouse and dragging it to another icon.

There are many block diagram icons for acquiring, processing, and outputting data. One may also create icons that interact with object code compiled in other computer languages. This would be used, for instance, to interface LabVIEW with instrument drivers that directly acquire data or control motors. We used LabVIEW's networking VIs to create a Remote Procedure Call (RPC) VI that sends commands to the vxWorks program, and used Transmission Control Protocol (TCP) VIs to stream the incoming data to a 2D graph. We also have the option of saving the data to a file or printing the front panel with its results.

\section{EPICS}

The Experimental Physics and Industrial Control System (EPICS) was developed at Los Alamos National Laboratory as a set of real-time control and data acquisition tools for particle accelerators. Particularly useful features for the distributed realtime system that controls the ALS are: the ability to monitor and control machine variables from any controller (as long as it has permission to do so), broadcasting the value of any variable to a given list of controllers, and event-driven data flow. This last feature means that a programmer can set up a block of code to execute only if a particular variable changes, instead of constantly polling its value (which wastes valuable CPU time).An experimenter can design a software model of the way his instrumentation operates using the concept of a state sequencer. For instance, we needed to initialize certain registers and buffers on our dsp board and laser axis board, then start data acquisition, and then display the results on a graph. Using state notation language (SNL, a small subset of the $\mathrm{C}$ language, with some database -specific commands added), we set up the sequence of events; non-SNL code (i.e. standard $C$ that is not part of $S N L$ ) is signified by using the escape 
character \%, and the two software modules are compiled separately and then linked.

Each database record is a structure comprising a set of fields called process variables, which are global variables available to the operating system for monitoring ongoing processes such as data collection, instrumentation status, and control. Thus we might declare a record called "Power" that is displayed in a window as a square labeled "ON" when its associated process is active, and changes to "OFF" if one clicked on it with the mouse; this would inactivate the process as well. Similarly, we might declare a record called "Data" and monitor its value; when that value changes, we can plot the new value on a Cartesian display. Thus the entire program is driven by changes in process variables, which allows for a much more efficient use of CPU time.

\section{Measurement and rectification of tilt in stage movement}

\section{Explanation of problem and data}

The purpose of the flexure stage is to move the mirrors in a straight line. While this may appear to be a simple task, it is one of the most important and demanding requirements. "Straight" means that the stage must not diverge from a straight line by an angle of more than $0.5 \mu \mathrm{rad}$ over its entire range of travel (this tolerance was derived above in the "Optical Component Tolerances" section above, p. 17). This requirement dictated the manufacturing tolerances of the flexure mechanism as well as the optics. 
Figure 17: Schematic of flexure mechanism, shown deformed. In the lab reference frame, the mirrors move in the $\pm z$ direction. The $x$-ray beam is directed toward the $+y$ direction and is split into two equal components that must remain coplanar through their separate paths, so they can interfere at the other end. Any phase difference must be due only to the linear movement of the mirrors and not to tilt around the $y$ axis (pitch). The stage is shown pushed to the far end of its traversal range. At zero path length difference the stage is centered and the flexures are parallel (see figure 10).

The flexure mechanism may be understood as two pairs of nested rectangles, each of which may be deformed into a parallelogram. One such pair of rectangles in figure 17 are $A B D C$ and EFHG. The outer rectangle described by the hinges $A B C D$ in figure 17 is broken out in figure 18. 
Figure 18: Schematic for tilt analysis. This is a perspective drawing of the outer rectangle $A B C D$ in figure 17, above. The stage travels in the $\pm z$ direction. The angles $\alpha$ and $\beta$ are measured between the axes of two neighboring hinges (which are supposed to be parallel) in the $x y$ and yz planes, respectively. In the figure, the two leftmost hinges should both be parallel in the xy plane to the coordinate measuring machine's x-axis. $\alpha_{1}$ is the angle of the rear axis and $\alpha_{2}$ is the angle of the front axis with the $x$-axis. The total angle of these axes relative to each other is $\alpha$. Similarly, $\beta$ is the total angle between the two front hinge axes, measured in the $x z$ plane parallel to the $x$-axis. Pitch error would correspond to rotation around the $y$ axis, and is primarily due to nonzero $\beta$ (see text).

We know from simple beam theory that a cantilever (a stiff but flexible thin rod, constrained at one end) of length $L$ may be modeled as a couple of length $2 \mathrm{~L} / 3$ and a torque centered at the pivot point. This model is valid only for small displacements of the cantilever from its equilibrium position. A double cantilever 
(constrained at both ends) may be modeled as two cantilevers whose "free" ends coincide with the center point between the two constrained ends.

The following analysis follows that of A.E. Hatheway in his paper on the alignment of flexure stages. ${ }^{23}$ Hatheway considered a flexure system consisting of two thin rectangular flexures supporting a rigid rectangular table. He analyzed the effects of nonparallel neutral axes (nonzero $\alpha$ in figure 18), nonparallel principal axes (nonzero $\beta$ in the figure), unequal span lengths, unequal flexure lengths, and driver misalignments. Of the above possible causes of tilt errors, our design eliminated all except the first two from contributing significantly to stage pitch for the following reasons: unequal lengths of the flexures or spans between them produce yaw in our system; rotation in this direction should not produce deflection of the beams because the mirrors act as pentaprisms -- if the beam strikes one at a more acute angle than ideal, it will strike the other mirror at an equally obtuse angle, canceling the error. Driver misalignments were compensated by adding the flexure joints in the connecting rod and adding a mechanism for adjusting the push point.

Roll and yaw produce negligible errors, so we consider only pitch error, or rotations around the $y$-axis $\left(R_{y}\right)$. "Inner" and "outer" rectangles refer to the rectangles EFHG and ABDC and their analogues MNPO and IJLK on the other side of the stage. The following equations for $\mathrm{R}_{\mathrm{y}}$ are taken from Hatheway; ${ }^{24}$ the values are from our stage. Nonparallel neutral axes $\mathrm{R}_{\mathrm{y}}=\frac{-3 \alpha \mathrm{T}_{z}^{2}}{4 \mathrm{SL}}$

$$
\begin{aligned}
& =\alpha \frac{-3(0.75 \mathrm{~cm})^{2}}{4(16.13 \mathrm{~cm})(7.62 \mathrm{~cm})}=-0.00343 \alpha \text { Outer rectangle } \\
& =\alpha \frac{-3(0.75 \mathrm{~cm})^{2}}{4(9.98 \mathrm{~cm})(7.62 \mathrm{~cm})}=-0.00555 \alpha \quad \text { Inner rectangle }
\end{aligned}
$$

Nonparallel principal axes: $\quad R_{y}=\frac{\beta T_{z}}{S}$

$$
\begin{array}{ll}
=\frac{\beta(0.75 \mathrm{~cm})}{16.13 \mathrm{~cm}}=0.0465 \beta & \text { Outer rectangle } \\
=\frac{\beta(0.75 \mathrm{~cm})}{9.98 \mathrm{~cm}}=0.0752 \beta & \text { Inner rectangle }
\end{array}
$$

where 


$$
\begin{aligned}
& R_{y}=\text { pitch angle } \\
& \alpha=\text { angle between neutral axes } \\
& \beta=\text { angle between principal axes } \\
& T_{z}=\frac{\text { stage travel dis tan ce }}{2} \text { between inner and outer rectangles) } \\
& \mathrm{S}=\text { span between flexures } \\
& \mathrm{L}=\text { flexure length }
\end{aligned}
$$

Note that the coefficients for the angles $\beta$ exceed those of the angles $\alpha$ by about 13 times and are of opposite sign; if the error angles $\alpha$ and $\beta$ are approximately equal, then the total pitch will be dominated by error due to nonparallel principal axes, i.e. $\beta$. In fact, the $\alpha$ 's were much smaller than the $\beta$ 's, as shown below.

The error angles $\alpha$ and $\beta$ are calculated from measurements made by the Coordinate Measuring Machine (CMM) at LBNL. This instrument can measure position with a resolution of $3 \mu \mathrm{m}$. The flexure mechanism was set on the CMM's optical table and fiducialized to create a lab reference frame. (Fiducial marks are reference marks on the flexure mechanism whose positions are known. The rest of the structures (hinges, beams, frame) are then supposed to have known coordinates in a Cartesian reference frame, within their respective tolerances.) Then the positions of the top and bottom of the flexures were compared. The difference between the two divided by the thickness of the flexure (2.000") gave the angles $\alpha$ and $\beta$.

A worst-case calculation using Hatheway's theory gives the tolerance for the machining of the flexure mechanism. Using the case of nonparallel principal axes for the inner rectangle, we get 


$$
\beta=\frac{\text { tolerance }}{2.000 "}=\frac{\mathrm{R}_{\mathrm{y}}}{0.0752}
$$

or

$$
\text { tolerance }=\frac{(1 \mu \mathrm{rad})(2.000 " \quad)}{0.0752}
$$$$
=0.00003^{\prime \prime}
$$

However, the manufacturer could not give us this tolerance. The best he could do was to offer a tolerance of 0.0003 ". When the stage was measured with the CMM, we found that instead of meeting the requested tolerances of $0.0003 "$, the flexures were out of tolerance by as much as $0.0035 "$, and instead of the errors being randomly distributed among the sixteen flexures, the largest errors were in the same direction along the principal axes. ${ }^{25}$ When the angles from the CMM measurements were plugged into the Hatheway model, the calculated pitch agreed with the measured pitch to within $10 \%$.

\section{Measurement of pitch during stage motion}

We used a $\mu$-Radian Instruments MRA-240 autocollimator in making pitch measurements of the stage. It has a range of three meters and an angular resolution of 0.1 arcsecond, or about $0.5 \mu \mathrm{rad}$. An autocollimator works in much the same way as the Shack interferometer, described above. Referring to figure 19, a bright light serves as a source. (Although it need not be either coherent or monochromatic, it does have to be bright enough to traverse twice the distance between the beamsplitter and the test surface and interfere at the measuring reticle. The autocollimator we have uses an ultra-bright LED as its source.) The light is then transmitted through two condensing lenses that are used to maximize both the intensity and uniformity of the light directed through the projection reticle in focal plane $\mathbf{P}_{\mathbf{1}}$. 


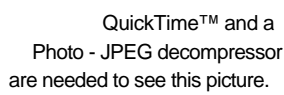

Figure 19: Autocollimator schematic. Outbound rays are indicated by solid lines, reflected rays by dashed lines. If $\theta=0$, the reflected rays would follow the paths of the outbound rays in reverse, reflect from the beamsplitter, and focus on the measuring reticle at $x=0$. For $\theta \quad 0$, the returning rays focus a distance $x=2 \theta f_{l}$ away. (Schematic courtesy Davidson Optronics.)

The beam is transmitted through the beamsplitter and collimated by the objective lens. After being reflected by a test mirror, light re-enters the autocollimator and is focused by the objective lens. The return image appears in sharp focus on the measuring reticle in focal plane $\mathbf{P}_{\mathbf{2}}$ after being redirected $90^{\circ}$ by the beamsplitter. Note that since the returning beam is focused by the same optics that collimated it in the first place, there will not be any dependence of the focal point upon the distance between the source and the image. The focal plane can be either an eyepiece or a CCD. The latter is used in our instrument.

If the test mirror has an angle $\theta$ with respect to the optical axis, the returning beam will focus at a point on $\mathbf{P}_{\mathbf{2}}$ a distance $X$ from the central ray, given by

$$
X=2 \theta f_{L}
$$

where $f_{L}$ is the focal length of the objective lens. From the equation it is apparent that $X$ is independent of the distance between the instrument and the reflecting surface. $X$ is the centroid of the spot on the CCD.

We used the autocollimator in measuring stage pitch by reflecting its light from the lower mirror mounted below the stage. (The mirror mount for the stage consists of a piece of aluminum about 4" $\times 2$ " x 1/2". There are two circular cutouts 1 " in diameter, 
in which two circular mirrors may be mounted. The upper mirror is coaxial with the driveshaft and the lower mirror sticks down below the stage at the same distance from the drive axis that the x-ray mirrors are above the axis. Originally, the lower mirror would have been used with a second laser positioning system to measure Abbé error, but we decided that was not worth the $\$ 15,000$ for another laser positioning system.) The upper mirror was needed for the laser interferometer to index position. Tilt measurements were made throughout the range of motion of the stage. We found that the stage tilted by more than $125 \mu$ rad over its range, as compared with the desired pitch of $0.5 \mu \mathrm{rad}$ (see p. 17). The tilt was linear with respect to distance traveled, and was quite reproducible.

\section{Rectification}

Discussion of the tilt in light of the Hatheway theory, and considering that the error angles $\beta$ were all in the same direction led to a suggestion: could we possibly correct for the dominant error by introducing a compensating tilt against the $\beta$ error? We decided to cut two slits between the frame and hinges (see figure 17). The slits were cut from the top of the frame to within $1 / 2$ " of the bottom, thus providing a hinge that could be opened up. Holes were drilled at each end of the two slits to accommodate taper pins. It was the taper pins that would provide the adjustment to the angular error. They were threaded at one end; as they were screwed in using a nut, the taper pushed against the upper end of the hole, opening the slit. The tilt was measured after each careful adjustment. By this method, the tilt of the stage was reduced from greater than $125 \mu \mathrm{rad}$ to less than $0.5 \mu \mathrm{rad}$ rms! The tilt adjustment has remained stable for over two years. 


\section{QuickTime $^{\mathrm{TM}}$ and a \\ Photo - JPEG decompressor \\ are needed to see this picture.}

Figure 20: Correction of stage tilt with taper pin adjustment

Blue trace -- underadjustment

Red trace -- overadjustment

Black trace -- correct adjustment 
QuickTime ${ }^{\mathrm{TM}}$ and a

Photo - JPEG decompressor

are needed to see this picture.

Figure 21: Expanded plot of stage tilt after correction. The pitch error has been reduced to $0.38 \mu \mathrm{rad}$, rms.

\section{Measurement and rectification of vibration and stick-slip \\ Explanation of problem and data}

Mechanical vibrations can be a problem if they produce Abbé errors in the frequency range of interest (at a stage motion of $\sim 15 \mu \mathrm{m} / \mathrm{sec}$ the $\mathrm{x}$-ray fringes are coming by the detector at $1 \mathrm{kHz}$ ). Stick-slip excites vibrational modes in much the same way as striking a bell with a hammer excites the bell's harmonics; a squarelike impulse in the time domain transforms into a sum of frequency peaks in the inverse frequency domain. Solving the stick-slip problem helps greatly in minimizing the vibration problem.

Abbé error in position measurement is the result of rotation of a rigid body around the point of measurement. In our case, we want to measure the position of the x-ray beam. The actual position measurement is made by reflecting laser light from a mirror whose surface is perpendicular to the axis of motion. The laser beam is collinear with the driveshaft axis. The x-ray beam strikes the x-ray mirrors about 67 $\mathrm{mm}$ above the driveshaft axis, so a pitch angle of $0.5 \mu \mathrm{rad}$ would produce a position error of $34 \mathrm{~nm}$. However, a constant error is not a problem; it would be subtracted 
out over the range of travel. What is a problem is the oscillation of position error about the "true" position. Such oscillations are measured as noise, and if the oscillation frequency is in the range of interest the vibration may significantly degrade the signal to noise ratio.

Stick-slip was measured by starting the piston moving at some reasonable speed, say $20 \mu \mathrm{m} / \mathrm{sec}$, and measuring the position as a function of time. The result is a line, but any small perturbations such as electronic noise, stick-slip, mechanical vibrations, and nonlinear optics are not visible unless the gross trend is subtracted. So that's what we did. Then we took a Fourier transform of the result and looked for peaks.

The following graphs illustrate our methods.

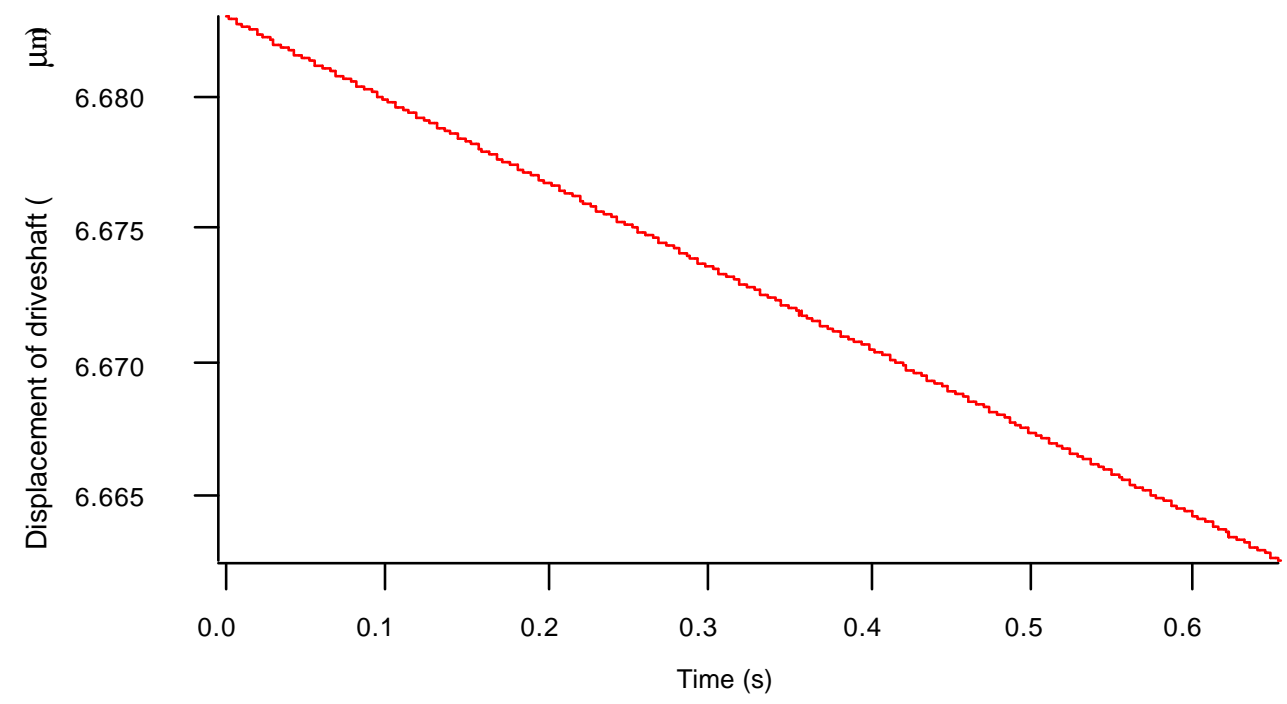

Figure 22: The stage is moving in a negative direction at $-31.3 \mu \mathrm{m} / \mathrm{sec}$, sample rate is 100,000 samples / sec, and 65536 samples were taken. 

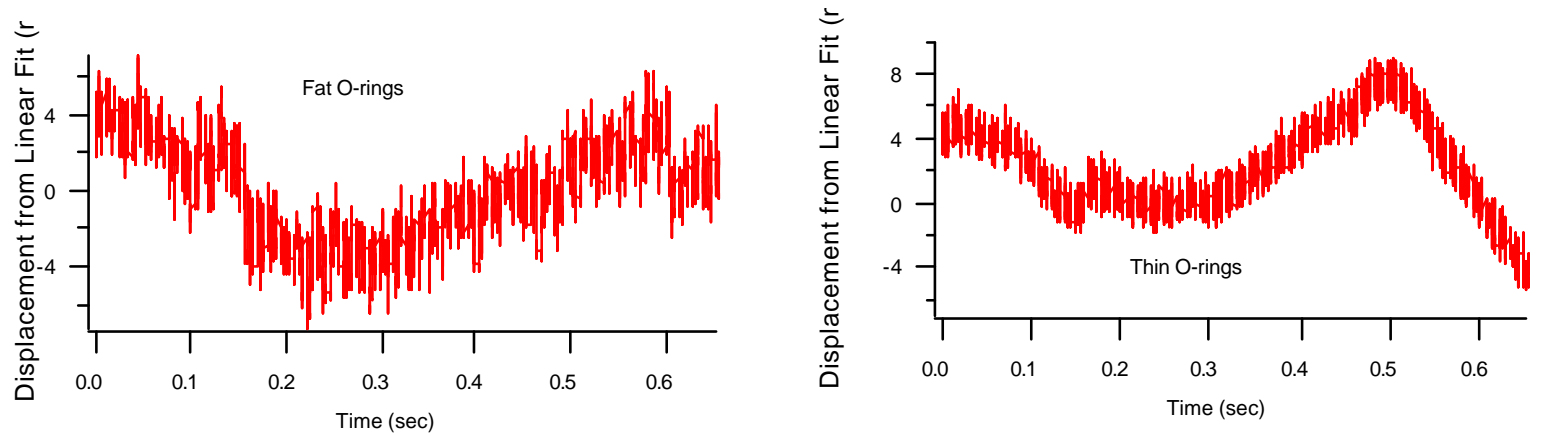

Figure 23: These are the same data (of figure 22) after they were fit with a line and that line was subtracted from them. The data on the left was taken when the fat (3/16" diameter) O-rings were installed as seals in the piston. We can see that the small perturbations have a peak-to-peak amplitude of 4 $\mathrm{nm}$. The data on the right were taken with thin (1/16" diameter) O-rings installed. The peak-to-peak amplitude is about $3 \mathrm{~nm}$.
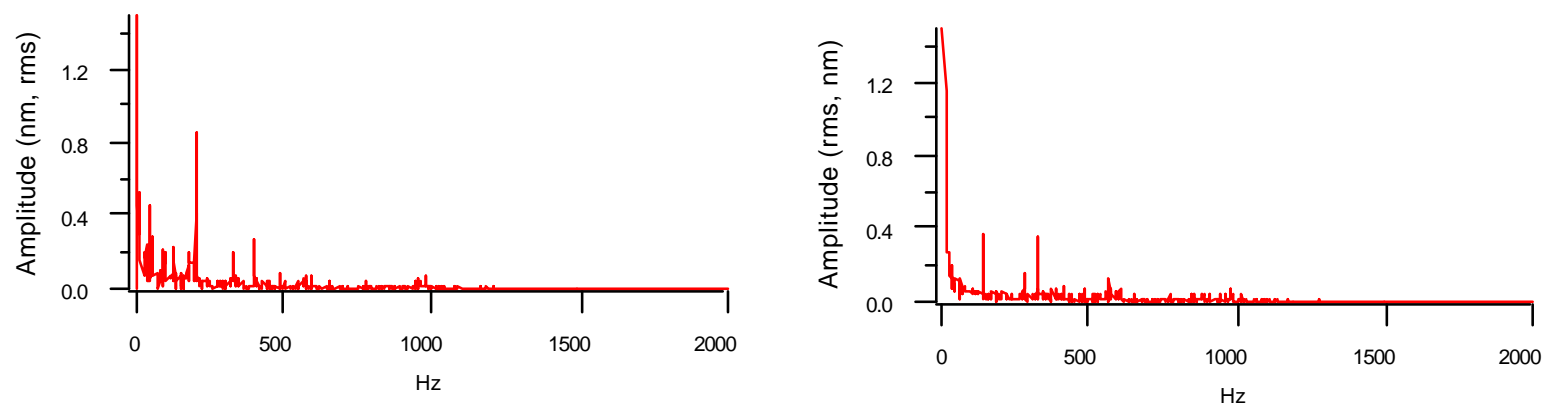

Figure 24: The greatest concern is the frequency of mechanical vibrations, which can affect the signal-to-noise ratio. A power spectrum of the linearized data is plotted here; we can see that the largest vibrations are below $500 \mathrm{~Hz}$, with amplitudes of less than $1 \mathrm{~nm}$ except for the one at $2 \mathrm{~Hz}$, corresponding to the stick-slip.

\section{Rectification}

Once the mechanical vibrations were identified we set to work reducing them. Two considerations drove our development efforts: natural vibrational modes and stickslip. One of the reasons for choosing cartwheel flexures and maraging steel was the stiffness of the flexures; this would force the locus of mechanical vibrations toward the high end of the frequency spectrum so they could be filtered out using digital filtering. This worked; most of the highest peaks occur above $10 \mathrm{kHz}$. 
The stick-slip was dealt with by concentrating on the points of contact between the driveshaft and the piston housing. Seals are necessary on a hydraulic piston to keep the hydraulic fluid inside, so they become one of the sliding surfaces, the other being the driveshaft. A much smaller piston and stage prototype was built before I joined the team, and one of the practical discoveries was the need to keep both sides of the seal wetted with lubricant. Therefore a plastic bellows was added on the outside of the piston to retain a reservoir of oil there. Buna (synthetic rubber) $\mathrm{O}$ rings, Teflon seals, and even no seals were tested for their stick-slip. The least stick-slip was observed when no seals were used (the driveshaft rode on the Teflon pressure plates used to keep the seals in place). However, the air pressure necessary to move the piston forced hydraulic fluid into the bellows, deforming them and threatening to rupture them. The prospect of mineral oil squirting all over the $x$ ray optics suggested a different course of action. The Teflon seals did not work either. Teflon has a very small coefficient of friction, but it is not elastic, so its sealing pressure was provided by a loose coil of spring steel. The added pressure produced unacceptable stick-slip. We settled on thin O-rings, 1/16" thick. The above graphs compare the differences between fat and thin O-rings. It can be seen that the thin O-rings produce less than half the amplitude of stick-slip of the fat $\mathrm{O}$ rings., and many fewer peaks are seen.

\section{Alignment of the beamsplitters}

The alignment of the beamsplitters with the mirrors was a technical problem. Two attempts were made to solve it. The ideal goal of alignment is to recombine the two beams of light at the focal plane (detector) without shear (transverse displacement) or relative angle. The practicable goal, to come as close as possible to the ideal, is dictated by the minimum visibility necessary to observe the features (absorption peak energies and their linewidths) predicted by theory. Excessive errors in shear or relative angle reduce the signal to noise ratio so that the interference fringes get washed out. These tolerances, derived earlier, are: shear $<200 \mu \mathrm{m}$ and relative angle $<1.0 \mu \mathrm{rad}$ (see p. 17, above). 
Provision for beamsplitter adjustments was made by holding each beamsplitter with three screws on one side, and three spring-loaded screws opposite them on the other side (see figure 25). Three points in space uniquely define a plane, and the placement of the screws was astutely chosen to follow an $x-y$ coordinate system where one screw is at the origin, the second along a line parallel to the long axis of the beamsplitter, and the third along a line parallel to the short axis. Thus by adjusting the second and third screws, the yaw and roll, respectively, of the beamsplitter could be adjusted independently. In practice, the first screw was turned a set amount, to translate a beamsplitter, and the roll and yaw were adjusted for the best interference pattern. Both beamsplitters had screws with 80 threads per inch pitches, so $1 / 4$ turn on the screw would move it by about $80 \mu \mathrm{m}$. The upstream beamsplitter had this simple mechanism; screws on the downstream beamsplitter were coupled to the beamsplitter via flexible steel levers so that 1/4 turn on the 80 TPI screw was scaled down to $\sim 1 \mu \mathrm{m}$.

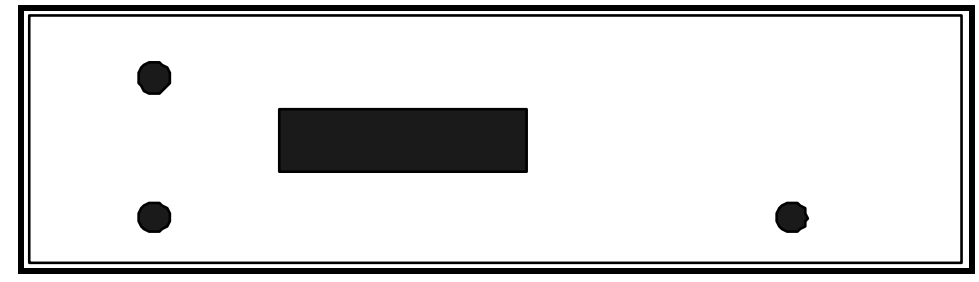

Figure 25: Contact points on a beamsplitter. The adjustment screws contacted the beamsplitter at the three black spots. Adjusting only the topmost screw changes the roll of the beamsplitter around the beam (which is being split at the grating represented by the black rectangle), while adjusting the rightmost screw changes the yaw of the beamsplitter.

We used HeNe laser light at first to provide a coherent source for coarse alignment, then substituted an incoherent neon lamp for finer adjustment. A CCD camera served as the human feedback amplifier. The person making the adjustment watched the CCD monitor and used a hex wrench to turn a screw. The angle and shape of the fringes give an idea of the direction one needs to adjust the screws. The aim is to get less than one fringe on the monitor. Horizontal lines mean the roll needs to be adjusted, and vertical lines mean the yaw needs adjustment. When the "best" adjustment is done, the camera is removed and the photodiode put in place. Then we take a spectrum of the light and look for good zero path length difference 
(ZPD) contrast. The shape is a sinusoid that grows to a maximum at ZPD and then dies out (see figure 26).

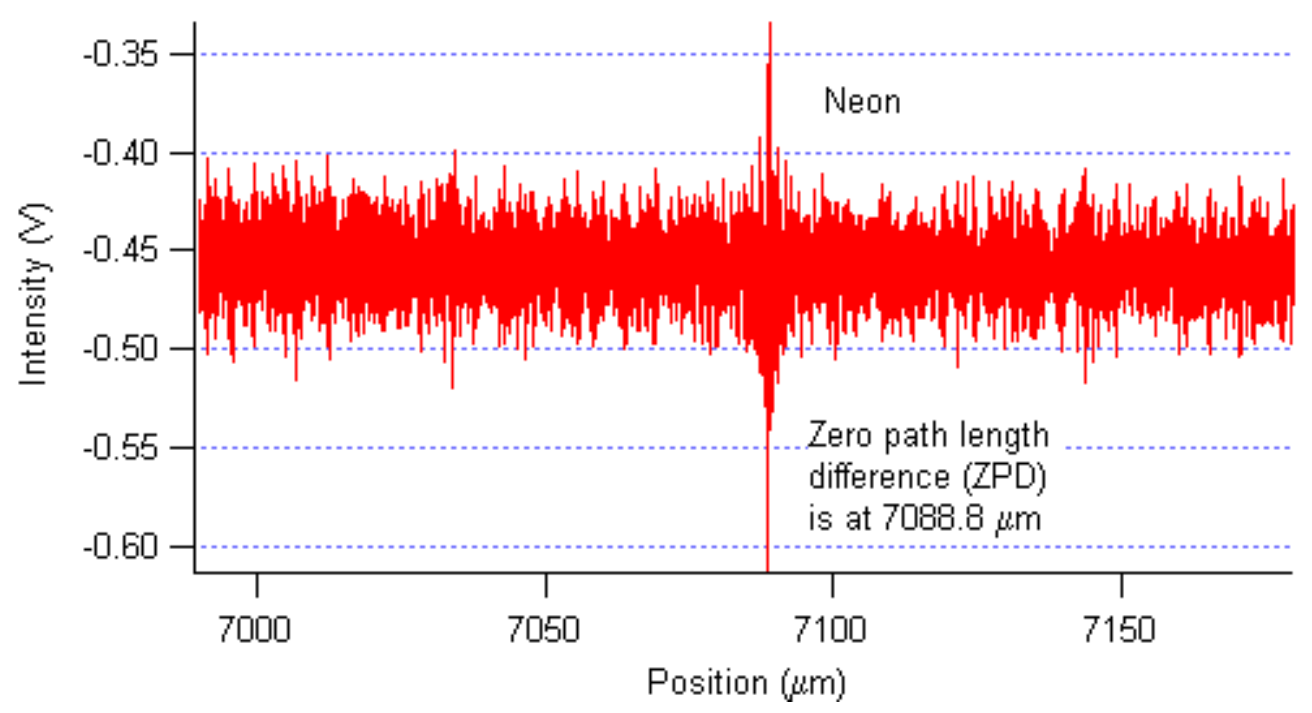

Figure 26 Alignment scan using neon light.

This manual adjustment served to get the beamsplitters well enough aligned to take a partially coherent neon interferogram. Within at most a few days, the alignment drifted off; the screws holding the beamsplitters' positions had moved enough to lose the alignment. Since pumping the vacuum chamber down to an acceptable pressure $\left(\sim 10^{-5}\right.$ torr $)$ takes several days, realigning the beamsplitters is often necessary under vacuum, and setting up and doing the experiment will take an unknown amount of time, this inability to hold alignment was unacceptable.

We rethought the adjustment mechanism and designed a computer-controlled alignment system consisting of picomotors and Linear Variable Differential Transformers (LVDTs) ${ }^{26}$. Picomotors are piezoelectrically-driven screws made by New Focus, Inc. When actuated, a picomotor turns its lead screw with an angular resolution of $<0.6 \mu \mathrm{rad}$, or $<30 \mathrm{~nm}$ linear motion with an $80 \mathrm{TPI}$ screw pitch. ${ }^{27} \mathrm{In}$ practice, the picomotor's "fingers" often stick to the lead screw when they should release, so the screw does not turn with the reliability of a stepper motor. We therefore added, with the help of Paul Denham of the Center for X-Ray Optics at LBNL, a position-measurement system based on Macro Sensors' LVDTs (model 
no. CD 375-050) and Analog Devices' AD698 LVDT Signal Conditioner. An LVDT measures position using three coils along a central axis, and a magnetically permeable tube within the bore of the coils called the core. The center coil is the primary winding, and the two coils on either side of it are secondary windings. The primary is energized with an $\mathrm{AC}$ signal, and its resultant magnetic field is coupled to the secondaries via the movable core. At its null position, the core couples equal magnetic flux to both secondaries, so the induced potential is zero. If the core moves closer to secondary \#1 than secondary \#2, more magnetic flux will be coupled to \#1 than \#2, and the induced potential $V_{1}>V_{2}$. The potential difference $V_{1}-V_{2}$ is then converted to a $D C$ voltage by the signal controller and sent to Hewlett-Packard's Data Acquisition/Switch Unit (model no. HP 34970A). This last instrument has an on-board digital multimeter to read the LVDT voltages, a switching unit to control the picomotors, and a GPIB interface so we can control it from our workstation.

This system provided very stable, accurate, and repeatable performance in aligning the beamsplitters. The system was calibrated with the autocollimator described above. The LVDTs have a sensitivity of $\sim 300 \mathrm{~nm} / \mathrm{mV}$. The coarse adjustment picomotors have a resolution of $14 \mu \mathrm{rad}$ on the yaw axis and $40 \mu \mathrm{rad}$ on the roll axis. The fine adjustment picomotors (attached to the flexible levers) have a resolution of $0.21 \mu \mathrm{rad}$ on the yaw axis and $0.27 \mu \mathrm{rad}$ on the roll axis. Figure 26 shows an alignment scan using incoherent neon light.

\section{Conclusions}

This paper has covered the design and construction of a Fourier transform soft x-ray interferometer at Lawrence Berkeley National Laboratory. The selection of the mechanical, optical, and data acquisition subsystems was driven by the requirements of high energy resolution and coherent recombination of the separated $x$-ray beams. In particular, introducing a $2 \mathrm{~cm}$. path length difference between the separated $x$-ray beams while maintaining coherence posed technical problems such as vibration, tilt correction and alignment whose solutions required experience and careful thought. This was a deceptively difficult instrument to build, 
and it was possible only with the combined efforts of knowledgeable engineers, scientists, and technicians.

The interferometer has met its specifications and held them for two years. In itself, this is quite an accomplishment, given the extremely tight manufacturing tolerances. It would appear the only thing left to do is to perform the autoionization experiment for which the instrument was designed. While an interferogram using synchrotron light has been produced, so far energy peaks have been observed only in the range of $0-10 \mathrm{eV}$. The scientists now working on the project are following several paths toward a solution. Among them are:

1. Adding fluorescent detectors throughout the path of the x-ray beam to track its progress, as an aid in alignment.

2. Replacing the mirror for switching the x-ray beam from the synchrotron to different endstations. (The other endstations use the beam in a direct line from the monochromator. Our endstation is th only one that uses the switching mirror, which could degrade the beam if it is corroded.)

3. Modifying the data acquisition software to take multiple scans and simplify the filtering of the raw data.

The goal of the interferometer, producing high-resolution double-excitation helium spectra, has exciting implications in many-body physics, chaos, and electron correlation theory. The successful conclusion of its construction will be just the beginning of a long and productive life.

\section{Appendix 1: Derivation of Geometric Factor Used in Calculating Path}

\section{Length Difference}

The following discussion pertains to movement of the mirrors with respect to the beamsplitters. In the diagram a mirror whose surface is at line 2 moves along the y axis to line $2^{\prime}$. Light entering the interferometer is reflected along line 1 at position $y_{01}$ and again at point $P$. When the mirror moves to $2^{\prime}$, the light continues to $\mathrm{P}^{\prime}$ before it is reflected. Thus the path the light travels before it arrives at center line $\mathrm{C}$ loses distance $\Delta \mathrm{x}$ and gains distance $\mathrm{r}$. 


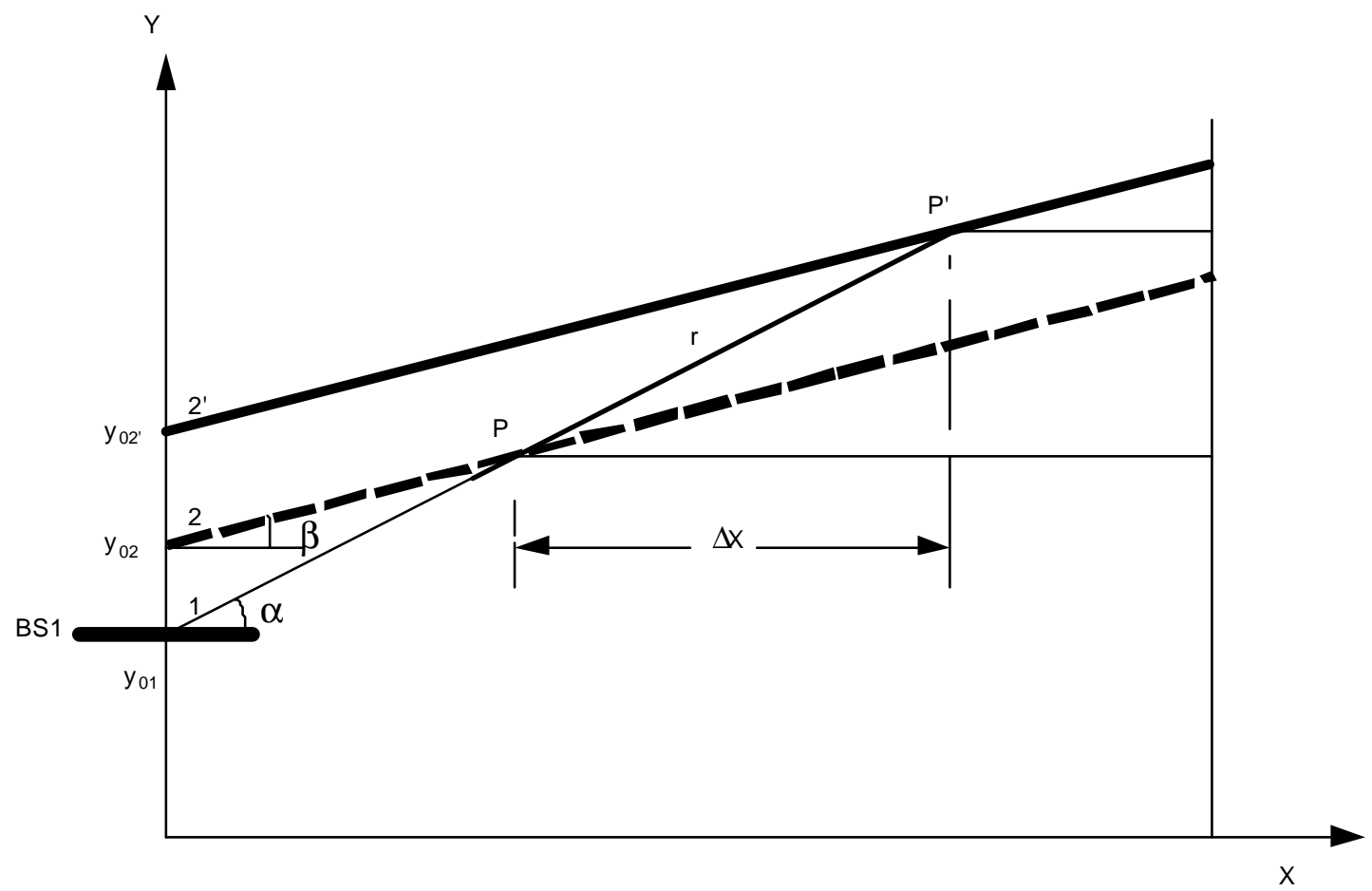

Figure A: Path length change due to moving one of the mirrors. The dashed line represents the position of the mirror before moving, the solid line represents the position afterwards. Unprimed variables before, primed after.

The equation for line 1 is

$$
\mathrm{y}_{1}=\mathrm{x}_{1} \tan \alpha+\mathrm{y}_{01}
$$

and for line 2 it is

$$
\mathrm{y}_{2}=\mathrm{x}_{2} \tan \beta+\mathrm{y}_{02}
$$

Now solve the two equations simultaneously to find the intersection $\mathrm{P}$.

$$
y_{P}=(\tan \alpha-\tan \beta) x+y_{01}
$$

Now if we move line 2 along the y axis to a new position $2^{\prime}$, we get for the intersection $\mathrm{P}^{\prime}$

$$
\mathrm{y}_{\mathrm{p}^{\prime}}=(\tan \alpha-\tan \beta) \mathrm{x}^{\prime}+\mathrm{y}_{01}
$$

Subtracting $A 1$ from $A 2$ gives

$$
y_{P^{\prime}}-y_{P}=(\tan \alpha-\tan \beta)\left(x^{\prime}-x\right)
$$

or

$$
\Delta \mathrm{X}=\frac{\Delta_{\mathrm{y}}}{\tan \alpha-\tan \beta}
$$


This $\Delta \mathrm{x}$ is the magnitude of the path length that is lost when the mirrors move by $\Delta \mathrm{y}$. The magnitude of the path length that is gained by the same motion is gained along the hypotenuse of the triangle $P^{\prime} P Q$, i.e. $r$ is given by (cf. figure $A$ )

$$
r=\Delta y\left(\frac{1}{\sin \alpha}\right)
$$

and the change in path length by

$$
\begin{aligned}
\mathrm{PLD}_{1} & =\mathrm{r}-\Delta \mathrm{x} \\
& =\Delta \mathrm{y}\left[\frac{1}{\sin \alpha}-\frac{1}{\tan \alpha-\tan \beta}\right\rceil
\end{aligned}
$$

where the subscript 1 indicates that this is the path length change for one leg of the light path through the interferometer. Referring to figure 4 in the text, we can see that the total path length difference between the two beams results from the lengthening of one beam and the shortening of the other beam by the same amount. Diagram A corresponds to the upper left quadrant of the diagrams in figure 4. Plugging in the values for our interferometer, $\alpha=20^{\circ}, \beta=10^{\circ}$, we get

$$
\mathrm{PLD}_{1}=0.342 \Delta \mathrm{y}
$$

and

$$
\mathrm{PLD}_{\mathrm{TOTAL}}=4^{*} \mathrm{PLD}_{1}=1.368 \Delta \mathrm{y}
$$

\section{Appendix 2: Software Descriptions}

\section{Dsp Program (dspacq.c)}

The dsp program, written in $\mathrm{C}$ and assembly, acquires data from three data channels (viz. position and two x-ray intensity signals), and sends them to the VME controller as one of several streams: raw or filtered, binned or unbinned, or normalized signal data. Raw data means a time series capture of signal or position. Filtered data are raw data processed by the dsp through a finite impulse response (FIR) algorithm to eliminate noise. Binned data can be represented as a signal vs. position plot; although they are acquired separately, the x-ray signal depends upon the stage position. We can pick a position bin size and average all the x-ray signals that fall into that bin. Therefore it is possible to obtain a higher position resolution than that published by the manufacturer. Normalized signal data are data from the position-varying $x$-ray channel divided by the data from the 
reference x-ray channel. This is done to eliminate changes in the x-ray signal external to the experimental setup, such as flux variations or motion of the beam on the detector.

The following description pertains to "dspacq.c" as it existed on 11/29/98. I will use the commenting in that version as section headers for the DSP's setup and operation. I will not describe variable declarations, allocation of memory, and other such housekeeping matters. DSP code itself is in boldface; my description is in normal type.

There are two functions defined in dspacq.c: "main" and "c_int05". "Main" sets up the various registers on the two VME carrier boards and then enters an infinite while loop. The while loop is only interrupted by the interrupt service routine (ISR) c_int05.

\section{/* Do Master Cycle in order to be the VMEbus master */}

\section{$\mathrm{i}=\operatorname{Read}\left(\mathrm{VME} \_\mathrm{CONTROL}\right) ; \quad \quad$ * Save VME master cycle register */ Write(VME_CONTROL, 0X00); /* Set VME master cycle register */}

This is necessary to acquire control of the VME bus. VME_CONTROL is a register in the VMEbus Interface Control (VIC) chip that, as one function, sets the control of the VME bus to be the VIC chip on the dsp carrier board instead of the VIC chip on the MVME167.

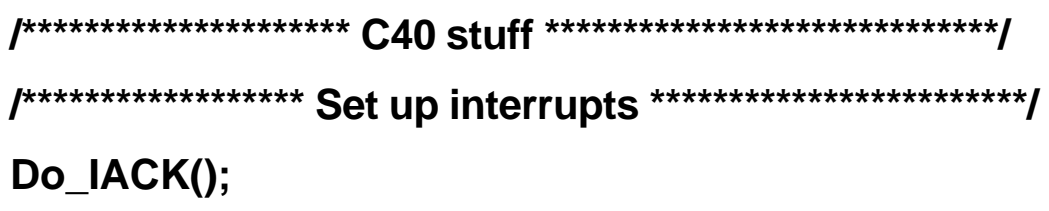

This is a macro defined in the header file that sends an "interrupt acknowledged" signal to the DSP control register. Since we have not yet started the program running, this action 
should not be necessary. However, the state of all hardware registers is undefined at pro-

gram startup, so they must be initialized to a known state.

set_ivtp(IVTP_DEFAULT);

for(i=0; i<0 $0 \times 40 ; i++)$

*(unsigned int *) $(0 \times 2 \mathrm{ffe} 00+i)=0$;

install_int_vector((void *)c_int05,0x05); / Set ISR to go when we get an interrupt *

The interrupt vector table (IVT) is a 64-word array in memory containing pointers to all the

available ISRs. The set_ivtp macro sets up this array at a page boundary, in this case IVTP_DEFAULT, defined to be a pointer to the location 0x2ffe00. The for statement zeroes that array, and the install_int_vector macro writes the pointer to the ISR c_int05 into the interrupt vector table.

\section{$l^{*}$ Set up IIF */}

Release_IIOF();

Enable_IIOF_CV2(2, "level"); /* Enable IIOF2 to be level-triggered unlatched interrupt line */

The interrupt flag register (iif) controls the character of the interrupts. There are three interrupt lines that may be configured as either interrupts or general-purpose I/O lines. The latter may have such functions as timing, motor control, or anything else that uses a TTL signal. An interrupt may be either edge-triggered or leveltriggered, and may either be latched into the interrupt flag register until explicitly released by a command, or released by a read or write of a data register (unlatched). Here, we first release the Interrupt/Input/Output lines for reconfiguration, and then set line IIOF2 to be level-triggered, unlatched. This function call also enables the interrupt. 


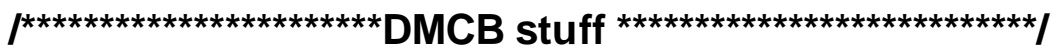

The Daughter Module Carrier Board (DMCB) is configured by writing to registers that are offset from the dBEX32 base address 0xB0000000. The first 64 words are used to configure the four Application ModulE Link Interface Adapter (AMELIA) chips that control the four Daughter Module (DM) sites on the DMCB. The next six words configure six registers that apply to the DMCB as a whole.

${ }^{*}$ Reset the AMELIA2's on the DM carrier board, then release them */

*DMCB_RESET_REG = 0x0f;

*DMCB_RESET_REG = 0x00;

$/{ }^{*}$ Reset the DM carrier board */

*DMCB_ISR_CTRL = 0;

*DMCB_XRDY_REG $=0$;

*DMCB_SYNC_REG = 0;

The above registers are four of the six general purpose register available on the Daughter Module Carrier Board (DMCB). The 4-bit reset register, as its name implies, resets the AMELIA2 chips that are mapped into it; the corresponding AMELIA2 is reset when the bit is set to 1 and then reset to 0 . The interrupt control register (ISR_CTRL) is an 8-bit register used to select which of the three interrupt lines, $\overline{\text { XINTA }}, \overline{\text { XINTB }}$, or $\overline{\text { XINTC }}$, will be used for each daughter module, $A, B, C$, or $D$. Each module is controlled by two of the eight bits; thus setting ISR_CTRL = 0x80 will assign $\overline{\text { XINTB }}$ to daughter module $\mathrm{B}$. The synchronization register allows a timer on one of the daughter modules (DM) to control the timing on any of the others. We are using only one DM, so this is set to zero.

/* Configure the DM Site's AMELIA2 (and therefore the DM) */ WriteUL(DMCB_SITE_A + AMELIA_CTR, D16DS_CTR); 


\section{WriteUL(DMCB_SITE_A + AMELIA_CMR, D16DS_CMR); WriteUL(DMCB_SITE_A + AMELIA_TMR1, D16DS_TMR1);}

The sample frequency of the $A D C$ is set by configuring three registers on the appropriate AMELIA2 chip. The user must first select the clock source and then prescale (divide) its frequency by some factor to arrive at the sample frequency. The User Control Register (CTR) is used to select the clock source and prescale its input into the Timer 1 (TMR1) register. Two clock frequencies are available from crystal oscillators on the DMCB: $12.288 \mathrm{MHz}$ (TCLK_0) and $11.2896 \mathrm{MHz}$ (TCLK_1). Bits 8 and 9 select the clock source: we use TCLK_0. Prescaling these frequencies to accommodate lower frequency DMs is accomplished by setting bits 6 and 7 . We use a prescaling factor of 1 .

The AMELIA Control Register (CMR) is used to configure sixteen control lines whose functions are determined by what DM is installed. Since the AM/D16SA has $A D C$ and DAC channels, they can be operated at different frequencies. Bits 4 and 5 of this register select the sample clock for the input (ADC) and output (DAC) channels. We use the same sample clock for the ADC and DAC, TCLK_0, so these bits are both set to 1 . Bit 7 is used to determine whether the current DM is the timer master of the other DMs, or a slave to another timer master. This provides synchronization of sampling. We have only one DM on the DMCB, so our DM is set as a master.

TMR1 uses the prescaling of CTR to determine the sampling frequency. Its value is given by the equation

$$
\mathrm{TMR1} 10=65537-\left(\frac{\mathrm{F}_{\text {clk }}}{\mathrm{F}_{\mathrm{s}}}\right)
$$

where $\mathrm{F}_{\mathrm{clk}}=$ Frequency of the prescaled selected clock

and $\quad F_{S}=$ Desired sample rate

This register can be set on the fly, and we do so whenever we want to change the sample frequency. The subtlety here is that TMR1 is an integer, not a floating point 
number. so the above equation truncates the result of the right side to an integer. The actual sample frequency is then given by

$$
\mathrm{FS}=\frac{\mathrm{F}_{\mathrm{clk}}}{65537-\mathrm{TMR} 1}
$$

We deal with this detail at the LabVIEW level: when the user inputs a desired sample frequency, the above calculation is performed and the actual calculated frequency is displayed for the user's contemplation.

\section{WriteUL(DMCB_SITE_A + AMELIA_CFR, D16DS_CFR);}

The configuration register is for configuring the data transfer interface between AMELIA2 and the DM. The manual did not explain this interface; it merely stated that the magic value $0 \times 8 \mathrm{dff}$ was required to establish valid data communications.

\section{WriteUL(DMCB_SITE_A + AMELIA_IMR, D16DS_IMR);}

The interrupt mask register dictates when an interrupt is sent to the C40 via the dBEX interface. An interrupt may be sent either when an input data register is full (i.e. an analog-to-digital conversion has been done) or an output data register is empty (i.e. a digital-to-analog conversion has been done). Setting bits 7 and 14, respectively, unmasks these two interrupts. We set the interrupt to be sent when an analog-to-digital conversion has been done.

\section{$/ *$ Configure interrupts */}

*DMCB_ISR_CTRL $\mid=($ XINTB_EN $<$ SEL_SITE_A);

*DMCB_ISR_CTRL |= (NO_INT_EN $<<$ SEL_SITE_B);

*DMCB_ISR_CTRL |= (NO_INT_EN $<<$ SEL_SITE_C);

*DMCB_ISR_CTRL |= (NO_INT_EN $<<$ SEL_SITE_D); 
Here we set the DMCB interrupt control register to configure interrupts for the four DM sites; site $A$ is the only one with a DM in it, so interrupt $B$ is enabled for it, and interrupts are disabled for the other three sites.

${ }^{*}$ Clear the FIFOS of invalid data */

d = ReadUL(DMCB_SITE_A + AMELIA_DATA0_IN);

d = ReadUL(DMCB_SITE_A + AMELIA_DATA1_IN);

WriteUL(DMCB_SITE_A + AMELIA_DATAO_OUT, 0X0);

WriteUL(DMCB_SITE_A + AMELIA_DATA1_OUT, 0X0);

The ADC and DAC data registers start out with undefined data in them, so they are read and written to clear them.

$\left.\right|^{\star \star \star \star \star \star \star \star \star \star \star \star \star \star \star}$ Clear all interrupts

I* On DMCB */

$$
\text { d = ReadUL(DMCB_SITE_A + AMELIA_ISR); }
$$

Clear the AMELIA2 interrupt service register by reading it.

/* Global Interrupt Enable and Cache Enable */

CACHE_ON();

INT_ENABLE();

Back on the dsp, the last thing we have to do before entering the infinite while loop that is the usual operating mode, is to enable the instruction cache that holds up to 128 words of past instructions for faster processing, and to globally enable all interrupts coming into the dsp.

${ }^{\star \star \star \star \star \star \star \star \star \star \star \star \star ~}$ MAIN NON-INTERRUPT LOOP ${ }^{\star \star \star \star \star \star \star \star \star \star \star \star \star / ~}$

while(1) \{ 


\section{if(++counter1 > 100000) \\ \{TOGGLE(LED3);counter1 = 0;}

There are eight light-emitting diodes on the front panel of the DBV42 carrier board that the programmer may use for debugging. TOGGLE is a macro that exclusively ORs the value in the LED register with itself to switch its state. It is used here to indicate that the non-interrupt loop is running and isn't stuck.

\section{Description of one channel of data}

It may be helpful to future experimenters to explain the software by an example how data is acquired, processed, and sent up the chain of hardware to the user interface. I will choose the data channel "Raw Signal 1 binned by Raw HP." As the name denotes, the data in this channel consists of an array of numbers that represent raw (rather than digitally filtered) measurements of x-ray intensity from the interferogram detector (rather than the reference detector) that have been averaged into position bins whose size is set by the user. The position used for each signal datum is unfiltered data from the HP laser interferometer.

The use of flags is designed to minimize accesses (hits) on the VME bus. The interrupt service routine and non-interrupt loop run asynchronously, with the ISR having priority because of its time-critical task, namely taking data from their registers and putting them in their proper arrays before the next timeout. However, the non-interrupt loop has the responsibilities of processing the arrays and sending them on to the 167 before they can be refilled by the ISR. The tasks of the two blocks are therefore interdependent, so they must communicate with each other via flags.

Flags are also used to communicate from the program running on the 167 , VxServer, to the dsp program, dspacq. When the latter wants a particular set of data, it gets the array parameters from the LabVIEW user interface and writes them into local memory. Then it sets a local flag register whose bits correspond to the 
data streams that the dsp program can provide. During its initialization, VxServer has already mapped local memory addresses into VMEbus addresses, so dspacq knows where the flag registers and data arrays are. (VxServer could also write its flag register to the dsp, but that produces bus conflicts when the dsp also hits the VMEbus while doing something else.)

The order in which the flag registers are tested is important; testing registers locally is faster than across the VMEbus.

One final note: the dsp has two separate buses; local and global. They access different areas of memory. The ISR fills arrays in one area, sets a flag, and starts filling the other area. The non-interrupt loop uses DMA to move data from the first area while the ISR is filling the other one. This is done to prevent bus conflicts on the dsp. I will describe only the code for the local array; the global array is serviced by similar code.

\section{/* signal 1 raw binned by HP Raw */}

if(S1HPLocalTest == FULL) $\{\quad$ /*local buffer is full?*

Here we test whether the local buffer flag has been set by the ISR (see below).

\section{if(channel_go\&S1HP_FLAG) $\left\{/^{*} 167\right.$ wants more binned signal 1 data *}

channel_go is the local copy of the data stream request register that is set by VxServer in its local memory. This register is read from the 167 in the ISR (see below). Each bit corresponds to a different data stream. S1HP_FLAG is a mask for "Signal 1 binned by Raw HP." A TRUE condition means that the 167 wants this data. 
if (ReadUL(VME_BASE+S1HP_BFLAG/4)==EMPTY) $\left\{/{ }^{*} 167\right.$ buffer empty? */

This test queries a flag in 167 memory that is set after an array of data has been written to a file that is accessed by LabVIEW for displaying the data.

\section{S1HPBinSize = $1.0 / \operatorname{Read}($ VME_BASE + (S1HP_BINSIZE/4));}

Get the bin size (i.e. distance in HP steps). Successive bins produce an index into an array. Each signal datum is acquired simultaneously with a position datum. We subtract the least position from the current position in the HP position array and divide the result by the bin size to get the index into the binned signal array. The current signal datum is then added to the other signal data at that index, and after the whole array has been thusly binned, the summed value at each index is divided by the number of points that have been summed to produce an average.

\section{LeastPosLocal $=$ 0xEFFFFFFF; \\ GreatestBinLocal = 0;}

Set the least position in the current position array and the greatest bin index to their extremes.

$l^{*}$ Find minimum position within safe distance of first position (assume positions are monotonically increasing $)^{\star} /$ for(i=0;i<SAFE_DIST;i++)

LeastPosLocal =(HPLocal[i] < LeastPosLocal) ? HPLocal[i] :

\section{LeastPosLocal;}


We use stage position to form an index into the binned signal array. Since arrays begin with index zero, we look for the least position so that we will always get a nonnegative number when we subtract it from the current position. We take position and signal data with the stage traveling only in the positive direction, so successive positions should form a monotonically increasing series. But the stage jumps around, and there is mechanical and electrical noise in the system, so we have picked an arbitrary safe distance from the zeroth position within which the least position should reside.

\section{${ }^{*}$ Bin local signal 1 data $*$}

for(i=0; i<RAW_ARRAY_SIZE;i++)\{

Bin $=$ (int)(S1HPBinSize * (HPLocal[i] - LeastPosLocal));

We enter the binning loop and calculate the bin into which we add the i-th signal datum. The reader may have noticed that when the bin size was read from the 167, above, that its inverse was calculated. The dsp performs a multiplication faster than a division, and it is important to maximize the processing speed of operations performed in loops.

\section{if(Bin $<$ RAW_ARRAY_SIZE $\& \&$ Bin $>=0) \quad\{$}

This condition eliminates glitches (i.e. Bin > RAW_ARRAY_SIZE or Bin < 0).

GreatestBinLocal $=($ GreatestBinLocal $>$ Bin $)$ ? GreatestBinLocal : Bin;

If the bin size is greater than 1, the binned array will have fewer elements than the corresponding raw array, so we have to calculate how many points to send on to the 167. This statement will find that number after going through the entire array.

\section{S1HPLocal[Bin] += S1Local[i];}




\section{++SamplesInS1HPLocal[Bin]; \\ \}}

Add the current i-th element of the raw signal array to the calculated bin element of the binned signal array. Increment the integer array that will be used later to average the signal.

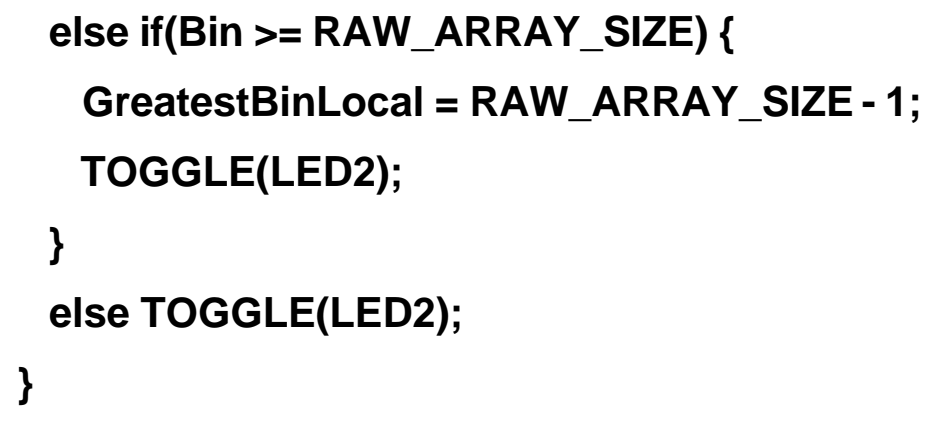

Toggle a front panel LED if there are any glitches, but don't include these points in the binned array.

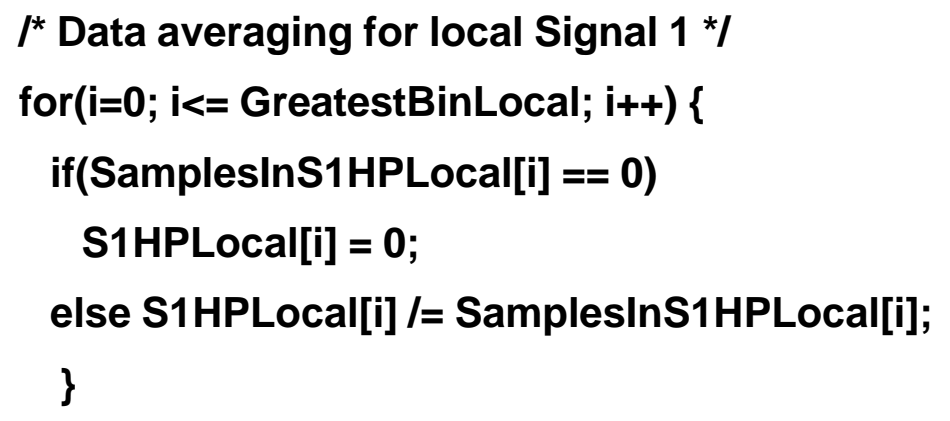

Summed data points within each bin are divided by the number of points to get an average.

Write(VME_BASE + S1HPSTARTPOS_PTR/4, LeastPosLocal); Write(VME_BASE + GREATESTBIN_PTR/4, GreatestBinLocal); 
movem((int)S1HPLocal, (int)(VME_BASE + S1PTR/4), GreatestBinLocal+1);

Send the least position in the current array, the number of points, and the x-ray signal data to 167 memory.

S1HPLocalTest = EMPTY;

for(i=0; $i<=$ GreatestBinLocal; i++) S1HPLocal[i] = SamplesInS1HPLocal[i] = 0;

vmeSetIntStatusID(VICVME_INTLVL,VICVME_INTVEC_S1HP);/* send an interrupt to the vmebus that the buffer is $\operatorname{moved}^{\star} /$

vmeSendAsyncInterrupt(VICVME_INTLVL);

Zero flags and buffers. Send an interrupt to the 167 controller to signal it that a buffer-full of data has been sent.

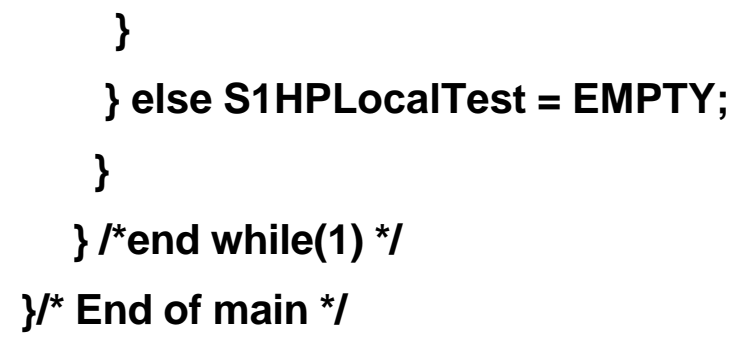


Read the interrupt status register, which must be done to reset the interrupt timer. Otherwise, the dsp would continually service the ISR and never reenter the infinite while loop.

\section{/* Acquire data */ \\ if (RawArrayFill==1) \{}

(Get global position and data points here; the code is similar to that for local data, described next.)

\} else \{

HPLocal[index]= HPreg_ptr->pos1_auto_smpl;

S1Local[index] = Read(DMCB_SITE_A + AMELIA_DATA0_IN);

The position datum resides in a 32-bit register on the HP laser axis board. It is accessed via the VME backplane. X-ray data resides in a 32-bit register in the AMELIA interface chip. It is accessed via the internal dBEX32 bus.

\section{S1Local[index] $<<=16 ; /^{\star}$ shift all the way left to preserve the sign bit ${ }^{\star}$ S1Local[index] >>=11;/* now drag the sign bit right, but leave 5 bits for resolution improvement */ \\ \}}

Even though data is sent as 32-bit integers, the ADC provides only a twoscomplement 16-bit integer. We can use the extra 16 bits to improve resolution when binning the data by left-shifting the data before averaging. The data is then sent to the 167 as 32-bit integers instead of floating-point numbers. Right-shifting drags the MSB to the right, so for a signed 16-bit number, we first left-shift by 16 bits to place the sign bit in the MSB, and then left-shift to drag that bit to the right. The result is a twos-complement signed 32-bit integer. 


\title{
if(++index $==$ RAW_ARRAY_SIZE) $\left\{l^{*}\right.$ handle full buffer */ \\ channel_go=ReadUL(VME_BASE + CHANNEL_GO_REGISTER/4); /* check which channels are enabled *I
}

A counter (index) is incremented every time the interrupt is serviced (or, equivalently, every time a data point is acquired). When the index reaches the size of the raw array buffers, we need to signal the non-interrupt loop that we have a full array and to start crunching numbers. Also we need to tell the ISR itself that the next time through it should store the data in the other area of memory. The channel go register is a bit-mapped flag register residing in controller (i.e. 167) memory. We set its bits at the 167 level whenever VxServer gets a request for data from the LabVIEW user interface.

\section{TOGGLE(LED7);}

Tell us that the interrupt is being serviced.

WriteUL(DMCB_SITE_A + AMELIA_TMR1, ReadUL(VME_BASE + ADC_RATE_DIVISOR / 4)); /* update sampling rate */

The procedure for setting the sample rate was described above. Here we read an address in 167 memory to provide runtime changes to the sample rate.

\author{
if(RawArrayFill $==1) \quad\left\{/^{*}\right.$ finished filling the Global array * \\ HPGlobalTest=FULL; /* set flags for data processing */ \\ S1GlobalTest=FULL; \\ S1HPGlobalTest=FULL;
}


The tasks of sending data to 167 memory and binning the data are done during the non-interrupt routine; this is where we tell the pertinent code blocks that the arrays are full. \{

if (HPLocalTest==FULL || S1LocalTest==FULL || S1HPLocalTest==FULL)

/* buffer overflow! */

TOGGLE(LED6);

vmeSetIntStatusID(VICVME_INTLVL,VICVME_INTVEC_RAWBUFOVFL); vmeSendAsyncInterrupt(VICVME_INTLVL);

\}

After an array of data that must be addressed via the global bus is operated upon and sent to the 167, its "array full" flag is reset. Here we check whether the corresponding flag for the local bus has been reset. If it has not been reset, the data has not yet been sent and will be overwritten by subsequent data. This could happen if the sample frequency is high and the dsp takes too long to process and send the data. We send an interrupt to the 167 and toggle a front panel LED to indicate a problem.

\} else \{

HPLocalTest=FULL; $\quad{ }^{*}$ set flags for data processing *

S1LocalTest=FULL;

S1HPLocalTest=FULL;

if (HPGlobalTest==FULL || S1GlobalTest==FULL \| S1HPGlobalTest==FULL) \{

/* buffer overflow! */

TOGGLE(LED6);

vmeSetIntStatusID(VICVME_INTLVL,VICVME_INTVEC_RAWBUFOVFL); 


\section{vmeSendAsyncInterrupt(VICVME_INTLVL);}

\}

Do the same as above for the local bus.

\}

RawArrayFill=RawArrayFill^1; /*toggle which array is being filled */

index = 0;

\}

\} ${ }^{*}$ end of c_int $05^{\star} /$

\section{VxWorks Program (VxServer.c)}

In a similar fashion to the dsp program, I will describe the behavior of only one datastream, "raw signal 1 binned by raw HP position." The main purpose of this program is to serve as an interface between the dsp program and the user interface. The user sends a data request from the LabVIEW user interface (running on a Sun workstation) to VxServer (running on the MVME167 controller, hereinafter referred to as the 167). Since the two programs reside on different computers, the request is sent via a Remote Procedure Call (RPC). The source code for this highlevel protocol can be automatically generated by using a Unix function "rpcgen." It is not described here more than by stating that the RPC task server on the local machine blocks CPU execution of local functions until it receives a properly constructed command sent from a remote computer.

Once the RPC for data acquisition is received by s1hpctrl_1, the mirror stage is moved to the start position, the pertinent parameters for this data stream are set to their values, and the S1HP flag in the channel_go register is set. The dsp continuously polls this register, and when it sees the flag immediately starts collecting data for that data stream. 
The dsp sends an interrupt to VxServer when one of its data arrays is filled. The applicable interrupt handler gives a semaphore (software "interrupt") to other code that is blocking CPU execution until the semaphore is taken. That code in turn does whatever processing is necessary and forwards the data array to the user interface on the Sun workstation.

The following are synopses of the functions in VxServer. The first three are tasks that are spawned in the vxWorks startup procedure. s1 hpctrl_1 and commandctrl_1 are RPCs invoked from the LabVIEW user interface, and S1HP_hndl is the interrupt handler that services the interrupt from the dsp.

init: initialize the dsp carrier board, get VMEbus addresses for local variables that the dsp needs to know about and send them to the dsp, configure the error interrupt handler.

dspacq_run: download the dsp Common Object File Format (COFF) program and start it running.

s1hpctrl_1: respond to an RPC from the user interface requesting "raw signal 1 binned by raw HP position" by opening binary files for buffering data, allocating memory, moving the stage to its start position, and setting the channel_go flag that the dsp monitors for determining which data to collect.

S1HP_run: set up the semaphore and dsp-to-167 interrupt, then go into an infinite while loop that waits for the semaphore. When it takes a semaphore, S1HP_run appends the data array to the binary file that the user interface reads. When the entire data set has been taken the program closes the binary files and frees memory.

S1HP_hndl: service the interrupt from the dsp indicating the latter has sent a buffer-full of binned signal 1 data to 167 memory by checking to see whether the entire set of data has been sent to the user interface. If all the data has been sent, the channel_go register is reset. Finally, the inter-process semaphore is given to S1HP_run. 
commandctrl_1: service RPCs requesting: changes in the sample frequency, stage position, or global data synchronization.

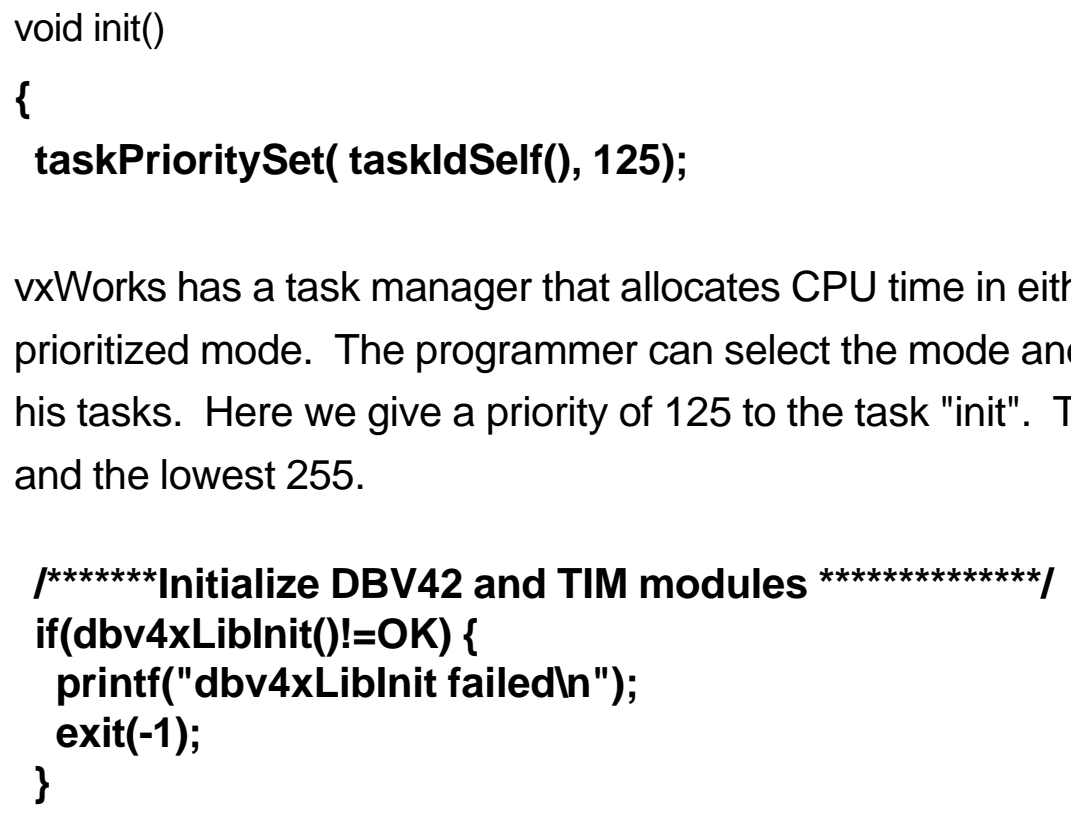
and the lowest 255.

vxWorks has a task manager that allocates CPU time in either a round-robin or a prioritized mode. The programmer can select the mode and priority given to any of his tasks. Here we give a priority of 125 to the task "init". The highest priority is 0

Initialize library of functions provided by Spectrum Communications for use with their dsp software.

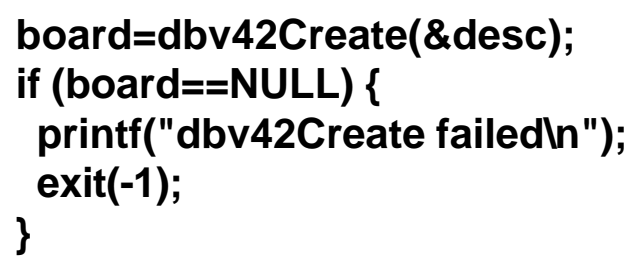

This function sets up the carrier board.

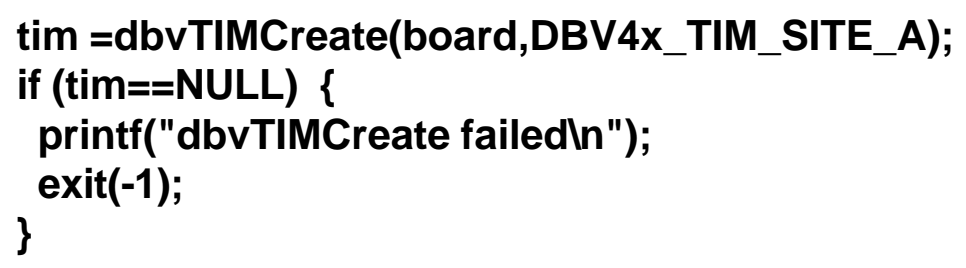


Make sure tim site $A$ is not already in use.

${ }^{*}$ Get VME addresses for several variables and write them to shared memory */

sysBusToLocalAdrs(HP10897A_addrs_type[HP_CARD], HP10897A_addrs_base[HP_CARD],(char $\left.{ }^{\star *}\right)(\& H P$ _regs_ptr));

/* adc rate divisor */

sysLocalToBusAdrs(VME_AM_EXT_USR_DATA, \&adc_rate_divisor,(char $\left.{ }^{\star \star}\right)($ \&tempintp)); dbv4xWriteSharedWord(board,\&tempintp, ADC_RATE_DIVISOR); printf("ADC_RATE_DIVISOR=\%dn",adc_rate_divisor);

/* first point of each HP array */

sysLocalToBusAdrs(VME_AM_EXT_USR_DATA, \&HPArrayStart,\&tempintp); dbv4xWriteSharedWord(board,\&tempintp,HP_ARRAY_START);

/* first point of each HP array */

sysLocalToBusAdrs(VME_AM_EXT_USR_DATA, \&S1HPArrayStart,\&tempintp); dbv4xWriteSharedWord(board,\&tempintp,S1HP_ARRAY_START);

$I^{*}$ number of points in each HP array */

sysLocalToBusAdrs(VME_AM_EXT_USR_DATA, \&BinNum,\&tempintp); dbv4xWriteSharedWord(board,\&tempintp,BINNUM);

${ }^{*}$ size of position bins */

sysLocalToBusAdrs(VME_AM_EXT_USR_DATA, \&BinSize,\&tempintp); dbv4xWriteSharedWord(board,\&tempintp,BINSIZE);

/* channel go reg */

sysLocalToBusAdrs(VME_AM_EXT_USR_DATA, \&channel_go_register,\&tempintp); dbv4xWriteSharedWord(board,\&tempintp,CHANNEL_GO_REGISTER);

The vxWorks operating system has many functions for accessing the VME bus. Two of them provide mapping of local addresses to VMEbus addresses (sysLocalToBusAdrs) or vice-versa (sysBusToLocalAdrs). We first declare local variables that need to be available to a board on the VMEbus, such as the ADC 
rate divisor. Then the sysLocalToBusAdrs function is executed to get the offset of that variable's address into the address block that has been set aside for VMEbus access. Lastly, the mapped address is written to shared memory on the pertinent VME board. In this case, dbv4xWriteSharedWord is a Spectrum Communications library call for writing to shared memory on the dsp carrier board. The latter has its own mapping of the VMEbus and uses the mapped address to write to and read from the address.

\section{${ }^{*}$ setup error-interrupt handler */}

\section{intConnect(INUM_TO_IVEC(VICVME_INTVEC_RAWBUFOVFL),RAWBovfl,(i} nt)0);

\section{sysIntEnable(VICVME_INTLVL);/“Enable interrupts ${ }^{*}$}

Here we connect and enable the dsp-to-167 interrupt for dealing with data array buffer overflows. The RAWBovfl is a pointer to the ISR that handles buffer overflows. VICVME_INTVEC_RAWBUFOVFL is a one-byte number $(=0 \times \mathrm{xD} 1)$ that we defined in the DSPVME.h header file. The programmer may define up to 256 interrupts for each of eight interrupt levels. The intConnect function writes the pointer to the ISR into an interrupt vector table that the CPU looks at whenever it receives an interrupt of the appropriate level. sysintEnable then sets a bit in the 167's interrupt service register that enables the interrupt level VICVME_LEVEL (=0x04).

\section{printf("End of Initın"); \\ \}}

int dspacq_run(void)

$\left\{I^{*}\right.$ Download the COFF file and Start it running * if(dbvTIMLoadObject(tim,TIM_FILENAME)!=OK) \{ printf("dbvTIMLoadObject failed $\backslash n$ "); exit(-1); \}

This function downloads a 'C40 COFF object file onto a TIM40 site. The entry address (if any) is remembered for later use.

else if(dbvTIMRun(tim,0)!=OK) \{ 


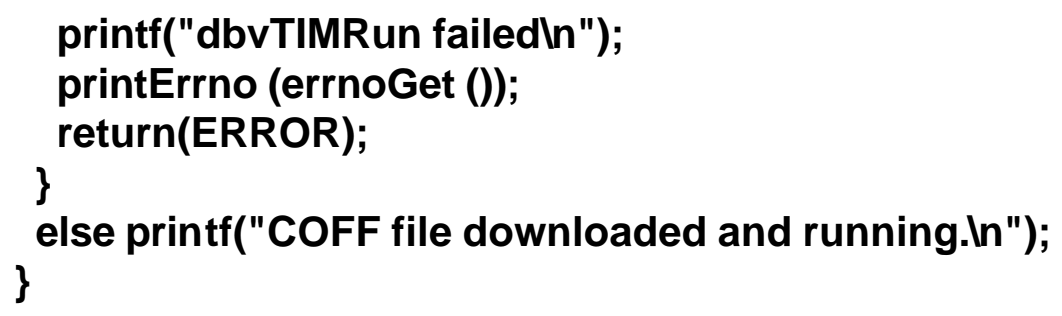

This function executes a program on the specified TIM site at the indicated entry point. If the entry point is 0 , then the entry point located in the last COFF module downloaded to that site is used.

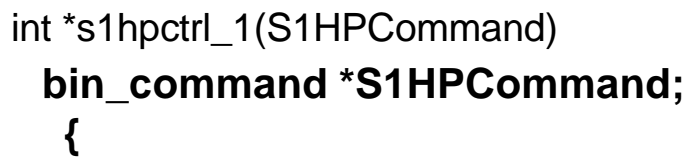

This remote procedure is called by the LabVIEW user interface. RPCs are a standard method designed by Sun Computer for executing functions between two computers. A port (111) is reserved on all computers using the standard. The computer acting as the server initiates its RPC service and then blocks (i.e. pauses CPU execution of the service until it receives a semaphore). The client sends a request, which consists of a program number, a version number, and a procedure number, to port 111 of the server. The server unblocks and processes the RPC.

\section{switch(S1HPCommand->execution_state) \{ case CAPTUREDATA: $/ *$ init the data collection */}

Here we decide which of the functions within the data channel "Raw Signal 1 binned by Raw HP position" to perform. There are two; CAPTUREDATA and ABORTCAPTURE. The former initiates data capture and then turns over control of data transfer from the DSP to the LabVIEW user interface to an ISR (S1HP_hndl) that checks whether all the data have been acquired; if they have not, S1HP_hndl gives a semaphore to the data-forwarding loop in S1HP_run. The latter writes the data buffer to a file on the Sun workstation, where LabVIEW reads and displays it. 


\section{S1HP=malloc(RAW_ARRAY_SIZE*sizeof(int)); ${ }^{*}$ allocate the data buffer * $/$}

Allocate memory for the data. Although the actual array will typically be much smaller than the size of the raw array buffer (the bin size would have to be smaller than the resolution of the position measurement, $\sim 3 \AA$, for this array to be larger than the raw array), this size is ample for our purposes.

\section{sysLocalToBusAdrs(VME_AM_EXT_USR_DATA,} S1HP,\&tempintp); /* tell DSP where buffer is */ dbv4xWriteSharedWord(board,\&tempintp,S1HPPTR);

Tell the DSP where the base address of the (local) data buffer is mapped into VME address space.

ret=0;

if (S1HP!=NULL) $\left\{l^{*}\right.$ any errors in allocations? */

Set the return value to zero (i.e. return OK). If things do not work out, this value will be changed later. Continue with this RPC only if we were able to allocate memory for the data buffer and open the binary data files. Otherwise, set the buffer pointer to zero and close the files (see below).

\section{BinSize = S1HPCommand->BinSize/HP_RESOLUTION; S1HPPoints = S1HPCommand->Points; \\ S1HPStart = S1HPCommand->Start; S1HPStop = S1HPCommand->Stop;}

The command received from the LabVIEW user interface is an ordered stream of bytes that we can separate into a structure of variables. Here we assign the structure members to local variables. This is done to isolate the command structure from local usage of the variable values: we do not want the values changing unpredictably in case a new command is sent.

$/^{*}$ go to start position ${ }^{\star}$

hydro_in();

while((HPArrayStart * HP_RESOLUTION) > S1HPStart - 100.0) \{ 


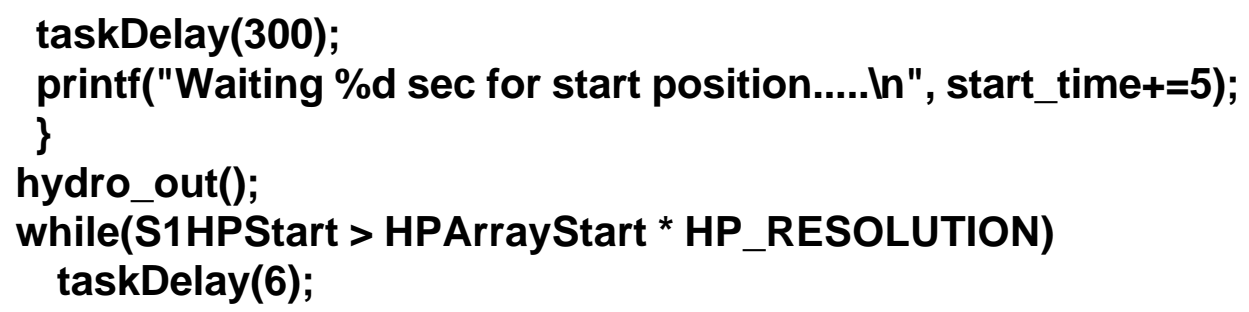

Move the stage to the start position. The commands "hydro_in" and "hydro_out" are drivers written by another member of our team (Scott Locklin) for moving the piston used to drive the stage back and forth. These functions set bits in a Xycom XVME240 digital I/O card whose outputs switch solenoid-driven pneumatic valves. This bit of code moves the stage away from the piston while reading the position every five seconds, until it is $100 \mathrm{~mm}$ past the starting position. The piston then reverses direction and samples the position at a faster rate $(0.1 \mathrm{sec})$ until it gets back to the start position.

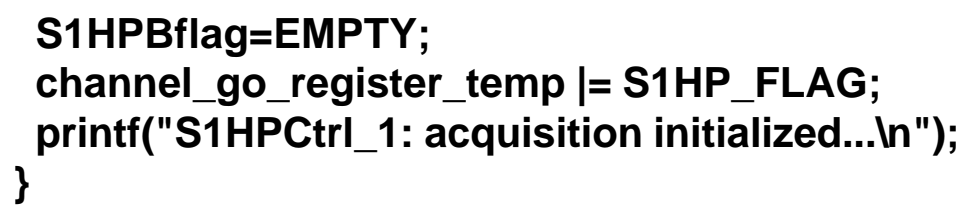

There are two indications to the DSP that we want data on this channel. The channel_go_register is a bit-mapped 32-bit integer that tells the DSP which of the twenty possible data streams are wanted. If a particular data stream is requested, its buffer flag tells the DSP whether the data buffer in 167 memory has been written to the binary file on the Sun workstation.

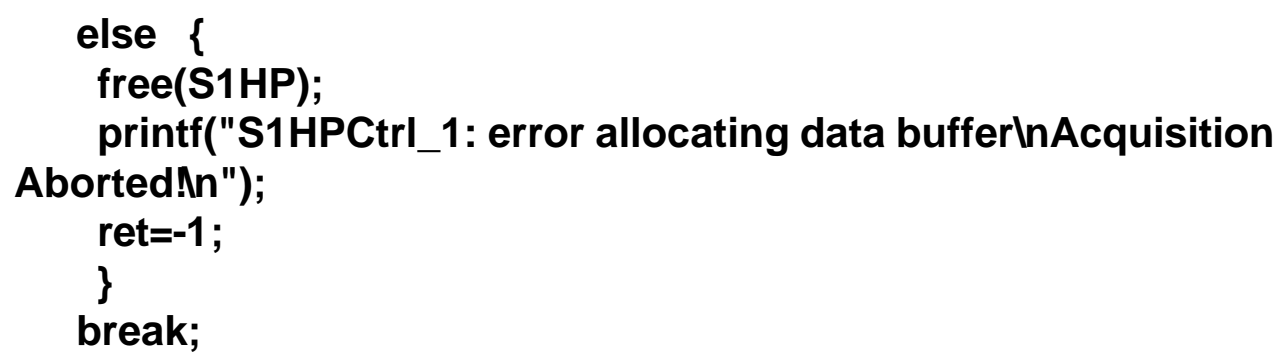

If there was an error in allocating memory, this is where we deallocate memory, print an error message, and return an error value to the calling routine. 


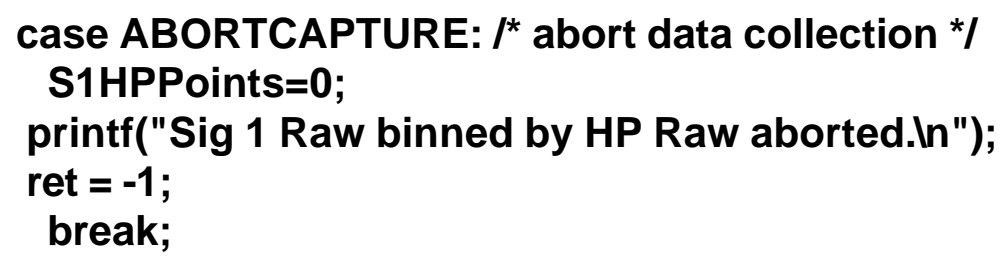

This is the RPC case that aborts data acquisition. It simply sets the number of points to zero, but as will be seen, this is sufficient to stop data capture and stop the stage from moving.

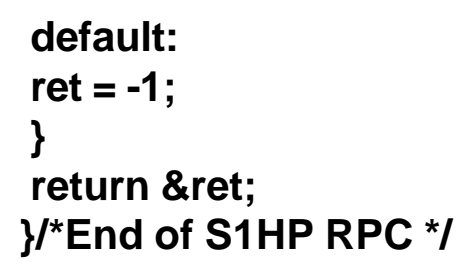

The default case is executed when no other case is. We actually return a pointer to the return value; this is a part of the RPC convention.

void S1HP_run() \{

S1HP_run is the function that does most of the work for this data channel. It waits for a semaphore that is given by the ISR that responds to an interrupt from the DSP. The DSP sends the interrupt when it finishes writing a buffer of data to 167 memory. When S1HP_run receives the semaphore, it writes the data buffer to a binary data stream that is associated with an Ethernet connection to the LabVIEW user interface on the Sun workstation.

\section{$/{ }^{*}$ task init */ taskPrioritySet( taskldSelf(), 105);}

Task prioritization is especially important in real-time operating systems. This means that the programmer can select which running programs - tasks - are more important than others. These tasks will preferentially receive CPU time when 
interrupts or semaphores from them are received by the CPU. Priority is a number from zero to 255 , with zero being the highest priority. The highest priorities have been appropriated by the system executive functions; we wouldn't want the operating system to stop functioning in order to service on of our tasks; it may not know how to start again! We have given our highest priority (100) to the port mapper task; it services RPCs, so we want to be able to send an abort command via RPC and have that override data acquisition or stage movement. Here we give a priority of 105 to the operational task for the "Signal 1 binned by Raw HP position" channel.

${ }^{*}$ store pointers to flag into dsp board */ sysLocalToBusAdrs(VME_AM_EXT_USR_DATA, \&S1HPBflag,\&tempintp); dbv4xWriteSharedWord(board,\&tempintp,S1HP_BFLAG);

Get the VME address of the S1HP buffer ready/not ready flag and send that to the DSP.

${ }^{*}$ setup semaphore for buffer filled interrupt message passing */ S1HPbufsem=semBCreate(SEM_Q_PRIORITY,SEM_EMPTY);

Create a binary semaphore that gives a software signal when the 167 CPU receives a "buffer full" interrupt from the DSP.

${ }^{*}$ init the interrupt handler for messages from DBV42 *

$I^{*}$ connect routine to interrupt $*$

intConnect(INUM_TO_IVEC(VICVME_INTVEC_S1HP),S1HP_hndl,(int)0);

Here we write the pointer to the interrupt service routine to the interrupt vector table; when the 167 receives an interrupt of a certain level (priority), it goes to the table for that level and uses the interrupt number to get the pointer to the ISR.

\footnotetext{
$/{ }^{*}$ always wait for the semaphore then process the buffer */ while(1) \{ semTake(S1HPbufsem,WAIT_FOREVER);
} 
We enter the infinite while loop that is the main operating mode of this task. We wait for the semaphore given by the DSP ISR after is fills a data buffer.

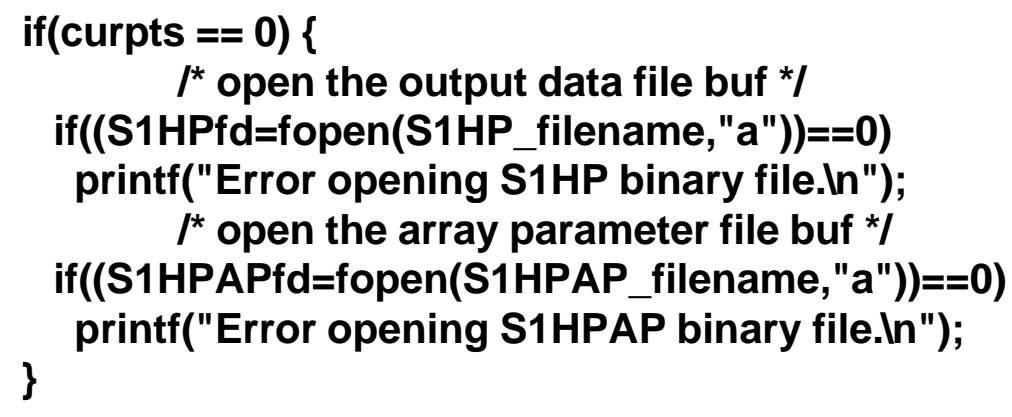

Open two files for writing binary data to the LabVIEW user interface on the Sun workstation. The first file, named S1HP.bin, is used for writing the data buffer. The second, named S1HPAP.bin, is used for writing the array parameters (viz. number of points in the variably-sized array and the starting position of the array) used in correctly indexing the LabVIEW display to the data.

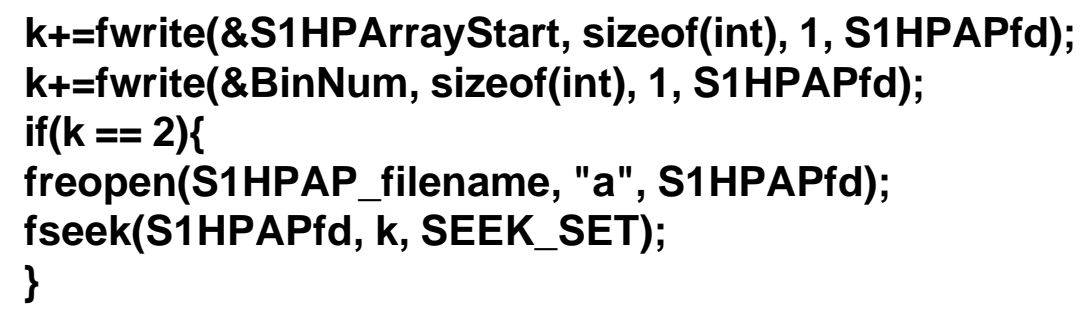

Write the starting position of the current signal data array and the number of points to the parameter file. These data are used to correctly place the signal data in the plot.

\section{$\mathrm{j}=\mathrm{BinNum}$;}

while $(\mathbf{j}>0) /{ }^{*}$ make sure whole buffer gets sent */ $\mathrm{j}$-=fwrite(S1HP+j-BinNum,sizeof(int),j,S1HPfd);

Write the signal data from 167 memory to the data stream buffers.

fflush(S1HPfd); fflush(S1HPAPfd); 
ioctl(S1HPfd, FIOSYNC, 0); /*sync to disk*/

ioctl(S1HPAPfd, FIOSYNC, 0); /*sync to disk*/ $^{*}$

Flushing the buffers means making sure the data remaining in them are sent to the stream. then send a command to the workstation to write the data from its input buffer to the hard disk. This action is normally performed periodically anyway by the operating system of the host computer, but we were having some problems with data overruns from the dsp-to-167 transfer. We determined that the problem lay in the 167-to-workstation transfer, specifically the Network File Service (NFS) was bogging down. Flushing the buffers and immediately writing the data to the disk interface helped.

curpts $+=$ BinNum;

S1HPBflag=EMPTY; /* signal to DSP that next buffer can be safely sent */

if $(($ channel_go_register \& S1HP_FLAG $)==0) /{ }^{*}$ end of data taking for now */ \{

fclose(S1HPfd);

fclose(S1HPAPfd); free(S1HP);

$\mathrm{S} 1 \mathrm{HP}=0$;

\}

\}$/{ }^{*}$ end while */

\}

FUNCPTR S1HP_hndl(int param)\{

S1HPBflag=FULL;

if(channel_go_register \& S1HP_FLAG) $\left\{/^{*}\right.$ if acquiring data on this channel */

if((S1HPPoints-=BinNum) $<=0)\{$

channel_go_register \&= S1HP_FLAG;

BinNum += S1HPPoints; /*only send enough points $/$

hydro_stop();

\}

semGive(S1HPbufsem);

\}

\}

西 
S1HP_hndl is the interrupt handler for the interrupt coming from the dsp. This interrupt signals that an array of binned data has been transferred to 167 memory. The handler sets the buffer flag S1HPBflag to FULL so the dsp knows when it checks that it will overwrite data in 167 memory. We then check to make sure we are actually supposed to be acquiring data on this channel, and then whether we have the requested number of points. If so, we reset the local data flag register and stop the piston from moving. Lastly, we give a semaphore to S1HP_run so it will send the data on to the workstation.

int *commandctrl_1(Command)

\section{command *Command;}

\{

commandctrl_1 services RPCs not connected with particular channels, viz. current position, ADC sample rate, and the global start command.

switch(Command->commandID) \{

case COMMAND_POSITION :

ret $=$ HP_posvel $(\overline{0}, 8)$;

break;

HP_posvel is a function from the HP software that polls the laser axis board's position register. We user it to give a running position measurement to a LabVIEW monitor.

\section{case COMMAND_ADCRATE :}

adc_rate_divisor $=$ Command->sample_rate_divisor;

printf("Changing ADC sampling rate to \%f kHz.In", 12288.0/(65537 adc_rate_divisor));

ret = 0;

break;

Change the ADC sample rate here. The divisor is calculated in LabVIEW and written to the local variable adc_rate_divisor. This is one of the variables whose local address was converted to a VME bus address during initialization. dspacq 
reads this value whenever the interrupt routine fills an array, and sets the sample rate accordingly.

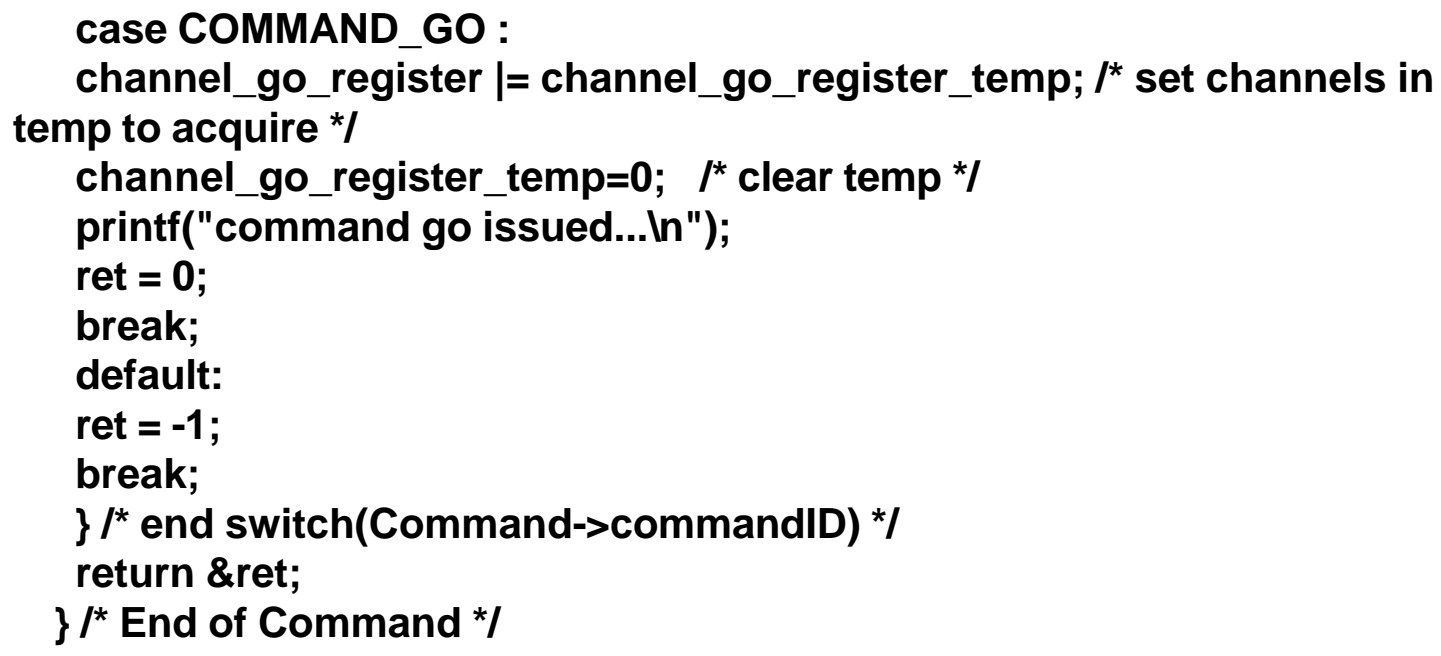

dspacq reads channel_go_register to find out which of the data channels need to be serviced. channel_go_temp is a local copy not visible to the dsp; its bits are set separately by the LabVIEW programs for the desired channels of data. The global start command is sent by Command.vi, and sets the channel_go_register equal to the accumulated channels in the temporary register. Thus data acquisition is synchronized for the selected channels.

${ }^{1}$ R.P. Madden and K. Codling, Phys. Rev. Lett., 10(1963) 516.

${ }^{2}$ J.W. Cooper, U. Fano and F.Prats, Phys. Rev. Lett., 10(1963) 518.

${ }^{3}$ L. Wu and J. Xi, J. Phys. B 23 (1990) 727.

${ }^{4}$ A.Macías, T. Martin, A. Riera and M. Yunez, Phys. Rev. A 36 (1987) 4187.

${ }^{5}$ M. Domke, K. Schulz, G. Remmers, and G. Kaindl, and D. Wintgen, "High Resolution Study of ${ }^{1} \mathrm{P}^{0}$ Doubleexcitation states in Helium", Phys. Rev A, 53, 1424 (1996).

${ }^{6}$ C. D. Lin, Phys. Rev. A 29, 1019 (1984). Much theoretical and experimental work has gone into the physics of autoionization of helium. While this phenomenon will not be dealt with further here, there are several good reviews of the literature, among them the paper by Domke, et. al., cited above, and this one. ${ }^{7}$ ibid.

${ }^{8}$ Huff, W. R. A, PhD Thesis, The University of California, Berkeley, LBL-38492(1996).

${ }^{9}$ Chamberlain, J., Chantry, G. W., and Stone, N. W. B., "The Principles of Interferometric Spectroscopy", (Wiley, New York, 1979).

${ }^{10}$ Howells, M., "Manufacturing Tolerances for the ALS Soft X-ray Interferometer", ALS Note, 5-27-93.

${ }^{11}$ I. S. Gradshteyn, and I. M. Ryzhik, "Table of integrals, series, and products", (New York : Academic Press, 1980).

${ }^{12}$ M. Born and E. Wolf, Principles of Optics, 7th ed., (Cambridge, Cambridge University Press, 1999), p. 546. ${ }^{13}$ op. cit., pp. 4-5.

${ }^{14}$ R. Duarte, M.R, Howells, Z. Hussain, T. Lauritzen, R. McGill, E. Moler, and J. Spring, "A Linear Motion 
Machine for Soft X-ray Interferometry", LBNL Report LBNL-40494, July, 1997.

${ }^{15}$ M.R. Howells, R. Duarte, R. McGill, "Properties of the Cartwheel-type Flexural Hinge", LBNL Report LSBL213, 1994.

${ }^{16}$ ibid., p. 6.

17"LBL BEAMSPLITTER QA DATA", Report of quality assurance, Rocketdyne Albuquerque Operations, June 2, 1995.

${ }^{18}$ The interaction of $\mathrm{x}$-rays with matter is a particular interest of the researchers at the Center for X-ray Optics at LBNL. See, for instance their website at http://www-cxro.lbl.gov.

${ }^{19}$ Facsimile communication from David Lunt of Photon Sciences to Malcolm Howells, November 11, 1994.

${ }^{20}$ Hewlett-Packard, "Laser and Optics Users Manual",(HP, Santa Clara, 1992).

${ }^{21}$ M. Krumrey, et al., Schottky type photodiodes as detectors in the VUV and soft x-ray range", Appl. Opt. 27, 4336, (1988).

${ }^{22}$ L.R. Canfield, J. Kerner, and R. Korde, "Stability and quantum efficiency performance of silicon photodiode detectors in the far ultra violet", Appl. Opt. 28, 3940, (1989).

${ }^{23}$ Hatheway, A. E., "Alignment of Flexure Stages for Best Rectilinear Performance", SPIE Proceedings, 2542, pp. 70 - 80.

${ }^{24}$ ibid.

${ }^{25}$ The raw CMM data is in a fax from Jerome Cummings to Rob Duarte, July 24, 1995. The analysis was performed by Rob Duarte and described to the author in a telephone call on April 28, 2000.

${ }^{26}$ S. Locklin, E. Moler, J. Spring, Z. Hussain, and M. Howells, "Progress in Soft X-ray Fourier Transform Spectrometry", from "Advanced Light Source Compendium of User Abstracts and Technical Reports 1997", D. J. Dixon, A. L. Robinson, A. Greiner, and C. Silva, eds., (LBNL, Berkeley, 1998).

${ }^{27}$ New Focus' online information at http://www.newfocus.com/Online catalog/6/145. 